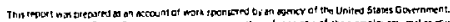

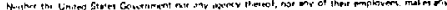

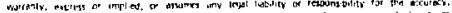

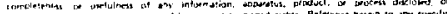

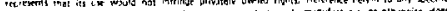

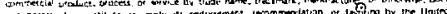

Metal-Atom Fluoressence from the Quenching of Metastable Rare Gases by Metal Carbonyls

william E. Hollingsworth

Materials and Molecular Research Division Lawrence Berkeley Laboratory

NOTICE

PORTIONS OF THIS REPC

has been reprotuced irc. copy to permit the broas ablits.

$$
\text { and }
$$

$$
\text { Department of Chemistry }
$$

University of California

Berkeley, California 94720

\title{
it leECiBLE. It
}

$\therefore$ siatiable,

Lsibile avail.

Abstract

A flowing afterglow apparatus was used to study the metal fluorescence resulting from the quenching of metastable rare gas states by metal carbonyls. The data from the quenching of argon, neon, and helium by iron and nickel carbonyl agreed well with a restricted degrees of freedom model indicating a concerted bond-breaking dissociation.

Tree bimolecular production of fluorescent iron atoms from the quenching of metastable argon states, primarily ${ }^{3} \mathrm{P}_{2}$, by fe(CO) 5 was studied in detail. The estimated total reaction cross-section was measured by absorption technigues to be about $1.6 \times 10^{-11} \mathrm{~cm}^{3} \mathrm{molec}^{-1} \mathrm{sec}^{-1}$, giving a $4 \AA^{2}$ cross-section.

Anomalously lcw formation rates for some long-lived states of iron were explained by intramultiplet relaxation. of the two possible quenchers, argon and $\mathrm{Fe}(\mathrm{CO})_{5}$, $\mathrm{Fe}(\mathrm{CO})_{5}$ was found to have a rate constant for quenching Fe states that was at or above the calculated hard-sphere 
cross-section. The distribution of levels lying less than $206 \mathrm{~cm}^{-1}$ apart closely matched a Boltzmann distribution. Fluorescence of metal states was observed from the quenching of the argon metastable states by $\mathrm{Cr}(\mathrm{CO})_{6}$, $\mathrm{MO}(\mathrm{CO})_{6}, \mathrm{CO}(\mathrm{CO})_{3} \mathrm{NO}$, and $\mathrm{Ee}\left(\mathrm{C}_{5} \mathrm{H}_{5}\right)_{2}$, and from the quenching of the helium metastable states by $\mathrm{Mn}_{2}(\mathrm{CO}) 10^{\circ}$

The reaction chemistry of the iron and nickel metal atoms was impractical due to the direct reaction of reagents with the parent carbonyls. No metal compound fluorescence was observed for the reagents tried. 
Acknowledgments

Thanks go to the following: John Winn, for his suggestions and ideas on this research; Dennis Hartman, for getting me started on this project, and working with me for the first two years; Doug Ray and the rest of the saykally group for the long-term loan of their Nicolet and $32 \mathrm{~K}$ PET; Michael Wax, for providing some compounds and offering suggestions from an organorccallic viewpoint; the Chemistry Department, for giving students after-hours access to their NBI Word processor which gave me the chance to pound this script into a recognizable form of English; Beth Schwehr, for providing a much-needed, critical reading of this manuscript; Professor George Flynn, for awaiting patiently (I hope!) the overdue arrival of his new Post Doc; and John Van curen for taking care of the loose ends after my departure.

This work was supported by the Division of Chemical Sciences, Office of Basic Energy Sciences, U.S. Department of Energy under contract No. DE-AC03-76SF00098.

The mentioning of the people listed above acknowledges only the most obvious of my debts incurred in my research and especially this writing. It seems a hopeless task to attempt to mention everyone and everything that $I$ am grateful to or enjoyed doing or knowing over a course of five years at Berkeley. To attempt to do so, and to fail, would seem to trivialize the whole experience. I hope everyone knows how I feel. 

iv 
Table of Contents

Acknowledgments. . . . . . . . . . . . . . . ii

Table of Contents. . . . . . . . . . . . . . . v

I. Introduction. . . . . . . . . . . . . . . I

A. The flowing afterglow technique. . . . . . . . I

B. The metal carbonyls. . . . . . . . . . . . 4

C. What's been done to iron carbonyl. . . . . . 8

Eigure 1-1. . . . . . . . . . . . . 12

II. Experimental. . . . . . . . . . . . . 1?

A. Metastable production. . . . . . . . . . 14

B. Introduction of reagent(s). . . . . . . . 16

C. Sbservation of product fluorescence. ..... 19

D. Data storage and manipulation. . . . . . . 21

Figures for Chapter II. . . . . . . . . . . 24

III. Absorption Experiments. . . . . . . . . . . 31

A. Concentrations of the argon metastable states . 31

B. Total rate constant for iron carbonyl. . . . . 33

Figures for Chapter III. . . . . . . . . . 38

IV. What Metal Transition Intensities Tell You. . . . . 38

A. Population and Rate Plots. . . . . . . . . 42

B. The statistical density of states model. . . . 48

C. Testing the model on iron and nickel carbonyl. . 54

Figures for Chapter IV. . . . . . . . . . . 62

V. Pressure Dependence of Long-lived Fe States. . . . 6\%

Figure 5-1................. . 77 
VI. Results with Other Carbonyls. . . . . . . . . 78

A. Argon and Chromium hexacarbonyl. . . . . . 80

B. Argon and Molybdenum hexacarbony 1. . . . . . 81

C. Helium and Dimanganese decacarbonyl. . . . . . 82

D. Argon and Cobalt tricarbonyl nitrosyl. . . . . 84

E. Argon and Ferrocene. . . . . . . . . 84

F. Other compounds tried. . . . . . . . . . 86

Figures for Chapter VI. . . . . . . . . . 87

VII. Reaction Chemistry of Metal Atoms. . . . . . . 100 VIII. Conclusions. . . . . . . . . . . . 103 References. . . . . . . . . . . . . 108 Table I: Rare Gas Metastable Energies. . . . . . . . 113 Table II: Physical Properties for some Metal Carbunyls . 114 Table III: Long-lived States of Iron. . . . . . . . 115 Table IV: Long-lived states of Nickel. . . . . . . . 116 Table V: Carbonyl Bond Energies. . . . . . . . . 117 Appendix: Computer Programs. . . . . . . . . . . . 118

A. "NICOLET $\rightarrow$ PET" . . . . . . . . . . . 118

B. "DATA PROCESS" . . . . . . . . . . . . . 119

C. "P\& $\&$ PLOTTER" . . . . . . . . . . . . 124 
Chapter I

Introduction

A. The flowing afterglow technique.

When a rare gas is excited in a d.c. discharge of around 300 volts, the effect of the perturbation upon the atoms is to promote electrons from the ground state configuration to many different higher orbits, thus creating a flow of atoms with a vast array of excited electronic energies. Most of these excited states find lower-lying electronic states to which they are connected by selection rules. The rapid relaxation of these states $v i a$ spontaneous fluorescence to lower states, a process occurring on the order of tens of nanoseconds, gives the strong and characteristic emissions associated with glow discharges.

Yet not all states are allowed to relax to the ground state. Due to selection rules, some states are in fact forbidden to fluoresce to the ground state on the same time scale. For the rare gases, the lifetimes of these "metastable" states are theoretically on the order of seconds (Stedman and Setser, 1972); however, they have other methods of relaxation fasier than spontaneous fluorescence available to them, such as energy loss through collisions with walls and other gaseous species. For example, for the argon metastable state at about one torr of total pressure, this collisional deactivation is on the order of milliseconds, so these states are still long-lived when compared 
to the higher states with allowed transitions. It is possible then to allow a period of time to pass such that all but the metastable states have relaxed, leaving these states as the only excited states. Experimentally, this is most easily accomplished by flowing the gas downstream from the discharge zone before reagents are introduced.

For the rare gases, a series of such metastable states exists. The ground states of the gases are all ${ }^{1} S_{0}$, corresponding to a closed outer shell of $(n) p^{6}$ (or $1 s^{2}$ for helium). The first excited states are created by promoting an electron from this closed shell to the higher $(n+1) \mathrm{s}$ level. For helium, there are two possible states produced: the ${ }^{1} s_{0}$ and ${ }^{3} s_{1}$. For the heavier rare gases, there are four: ${ }^{1} P_{1}$, and ${ }^{3} P_{0,1,2}$. With the selection rules $\Delta J=0, \pm 1$ but $J=0$ cannot go to $J=0$, there are two states for each rare gas that are forbidden to relax to the ground state: both of the He states, and the ${ }^{3} \mathrm{P}_{2}$ and the ${ }^{3} \mathrm{P}_{0}$ states for the others. These metastable states, and their energies are listed in Table I. Radon, lying below xenon in the periodic table, is also a rare gas, but its use is precluded due to its expense, its scarcity, and especially its radioactive character.

The method implied by the preceeding discussion is that of the flowing afterglow technique. Instead of having a rather intractable system for scudying collisional energy transfer consisting of the introduction of a reactant into the discharge zone with its myriad of excited rare gas 
states, the reactant is introduced downstream where at most only two excited states exist. In adcition, these two states lie very close in energy to one another and are very similar in character. For the heavier rare gases, the major energy carrier is the ${ }^{3} \mathrm{P}_{2}$ state. Due to the $2 \mathrm{~J}+1$ degeneracy, the state is at least five times more populated than the ${ }^{3} \mathrm{P}_{0}$ state. The ${ }^{3} \mathrm{P}_{2}$ state is even more favored since it also lies lowest in energy of the series of four clcse-lying energy states. This allows the auditional conversion of the ${ }^{3} \mathrm{~F}_{0}$ state to lower states, thus giving it an effectively higher quenching rate constant (Kolts and Setser, 1978 ). Experimental verification of this will be discussed in Chapter III.

The flowing afterglow technique was pioneered by Robertson (collins and Robertson, 1964; Prince et al., 1964). Since that time, a great amount of work has been done by many groups, owing to the relative experimental simplicity of the method and its rich applicability to problems of chemical interest. Setser has done extensive work in chemical applications and has come out with several very useful review articles (Stedman and Setser, 1972; Kolts and Setser, 1979 ).

With the series of rare gases then, one has a range of excitation energies available, a range that is chemically significant. The cost of using $\mathrm{Xe}$ and $\mathrm{Kr}$ can by mitigated by seeding a small amount in a flow of argon (Stedman and Setser, 1970; Piper et al., 1975). This reduction in cost 
is due to a very efficient transfer of energy from the higher metastable energy of argon to the lower metastable energies of xenon and kryptor. Even though most of the afterglow experiments have used the rare gases and especialiy argon, the method is not limited to the rare gases. The rumber of atomic and molecular species which have suitably long-lived excited states is quite large (Kolts and Setser, 1979). However, the use of molecular states, such as the $O_{2}\left(1_{\Delta}\right)$ state, requires the consideration of a range of vihrational energies in addition to the electronic. Also, in comparing to the rare gases, the situation is often more complicated due to the possibility of direct chemical reaction of the reagent with the parent gas.

Metastable care gas atoms are very reactive. They will react with essentially everything but lighter rare gas atoms. Many reactive channels are open, ranging from neutral excitation, dissociation, to ionization. Helium and neon energies lie weil above the ionization potentials for most gaseous species. As a result, ionization channels are relatively much more important for helium and neon than for argon. Also, with helium, there is potentially some concentration of $\mathrm{He}^{+}$and $\mathrm{He}_{2}{ }^{+}$in the flow (Kolts and setser, 1979), so the system is not as clean as it is for argon.

6. The metal carbonyls.

It's become a well-worn point to note the importance of iall metal clusters and their possible applications to 
problems of catalysis and surface chemistry (e.g. Schaefer, 1977; Muetterties, 1977; Muetterties et al., 1979). In adoition, metal atoms are very reactive species and should provide many interesting gas phase reactions. However, it's not the easiest thing in the world to study the specicroscopy and interactions of transitior. metal atoms and clusters in the gas phase. This is especially true for the "refractory" metals which are extremely high melters, existing at room temperature as solid chunks with virtually no vapor pressure; this class includes most of the transition metals. To get even l micron of vapur pressure for most of these metals requires heating to over $1300 \mathrm{~K}$ (Klabunde, $1980)$.

Most methods that do exist for the production of metal vapor are not very selective as to energy or cluster size. Methods using techniques such as high temperature firnaces are able to produce sufficient concentrations for study, but with a large variation in the energy of the atoms and molecules and an unspecified distribution of molecular size, and the necessity of working at high temperatures introduces its own unique shallenges. With the advent of lasers, more flexibility exists in methods of metal atom production, with some methods using ti:z sheer concentrated power of pulsed laser beams to :.:terally blast parts of a metal target into the gas phase (Dietz et al., 1981). Unpublished results from the same group suggest that clusters of iron atoms in the range of 10-30 atoms have been produced in a laser 
photolysis of a beam of iron carbonyl.

An alternate approach toward gas phase metal production is to take advantage of the physical properties of organometallic compounds. That is, some organometallic compounds are sufficiently volatile that the metal can be tricked into the gas phase at near roon temperature. Once there, in one way or another, the bonds with the surrounding ligands are broken to produce metal atoms, or perhaps finite metal clusters for organometallic compounds containing two or more metal atoms; however, the metal-metal bonds are generally weaker than the metal-ligand bonds, so it's not clear that cluster production is possible.

A class of compounds which springs to minis are the metal carbonyls, a class comprised of one or more metal atoms encapsulated among ligands of co molecules. They owe their collective existence to the "backbonding" that occurs between the metal and the $\mathrm{CO}^{\prime} \mathrm{s}$, a process so favored that carbonyl production for some is as simple as passing a flow of carbon monoxide over the finely-divided metal. The typical ligand-type forward bond involving the contribution of the carbon lone pair of the co to form a o-type bond with the metal is not sufficiently strong to explain carbonyl stability since $C O$ is a very poor base. The extra stability occurs from the additional back donation of some of the $d$ orbital population of the metal to form a r-type bond with an empty co antibonding orbital.

Many of the carbonyls are very volatile. Fe(CO) 5 and 
$\mathrm{Ni(CO)_{4 }}$ are both volatile liquids whereas many of the other mononuclear carbonyls are volatile and easily sublimable solids. Polynuclear metal carbonyls, such as $\mathrm{Fe}_{2}(\mathrm{CO})_{9}$ and $\mathrm{Mn}_{2}(\mathrm{CO})_{10}$, are generally less volatile solids, but still with more vapor pressure than the metal and often sufficiently volatile to get an adequate concentration in the gas phase, especially when the pressure is boosted with heating. Many of the carbonyls decompose easily with heating; in fact, in our laboratory, pyrolysis turned out to be "a very useful and easy way of disposing of extra Fe(CO) 5 ; only very durable iron crystals remained (Horak, $1982)$.

The fhysical properties of the carbonyls are not extremely well-documented and the information that does exist is very scattered. An incomplete compendium giving a taste of the physical properties for some of the smaller carbonyls is listed in Table II. The list could be made a lot longer by considering similar classes of organometallic compounds; that is, a whole classes of compounds exist containing a mixture of $c 0$ and other ligands such as No, alkanes, and the cyclopentadie:yl moiety, $\mathrm{C}_{5} \mathrm{H}_{5}$. The table of just carbonyls gives an indication of the variety of compounds that exist. The structures of some of these carbonyls are shown in Figure 1-1.1

Also not well-documented are the toxicities of the

1The references used for Table II and Figure 1-1: Abel and Stone (1969); Cotton and Wilkinson (1972); (1973); and the 1982 Alfa Catalog. 
carbonyls. Always present is the hazard of the possible release of carbon monoxide molecules. Iron carbonyl is moderately toxic. However, nickel carbonyl, one of the original suspects in Legionnaire's Disease, is rated dangerous in the part per billion range and is very difficult to handle safely due to its high volatilicy and its heaviness which makes it hard co exhaust. Its effects can be either acuce from the combined effects of co and nickel on the system, sr, more slowly, it can be a long-term cancer risk. For these reasons, $\mathrm{Ni}_{(\mathrm{CO})_{4}}$ was not used as extensively as was $\mathrm{Fe}(\mathrm{CO})_{5}$. When it was used, it was used in very low amounts and vented from the pump into an exhaust system.

C. What's been done to $\mathrm{Fe}(\mathrm{CO})_{5}{ }^{*}$

It's been known for some time that iron atoms can be produced from the flash photolysis of iron carbonyl. With the large amount of energy available in such a method, it's not surprising that atoms would be the result, However, it's not obvious that if one were to feed lesser amounts of energy into the carbonyl, still greater than, but on the order of, the total bond energy, that there would be an avenue roward metal atom production or fluorescence. Iron carbonyl is a Large enough molecule that, with its many available degrees of vibrational and rotational freedom, it would be more likely that a great deal of the energy would be lost in these modes on a time scale much faster than 
spontaneous fluorescerice.

The first indication that the energy might go different ways was an afterglow experiment where unexpected metal fluorescence was seen from the interaction of active nitrogen $\left(A^{3} \Sigma\right)$ with iron and nickel carbonyl (Brennan and Kistiakowsky, 1966). In fact, this process was discovered accidentally; no $\mathrm{Ni}(\mathrm{CO})_{4}$ was officially present, but was accidentally formed by the interaction of co at a high pressure with a nickel alloy. Not having sufficient energy in this nitrogen state to break all of the carbonyl bonds in one step, the metal atom production was explained by a stepwise loss of carbonyls from multiple collisions with the active nitrogen.

Next, in our group, using a flowing afterglow system providing metastable rare gas atoms, the interaction of iron carbonyl with Ar" was tested. The very surprising result was a strong bluish flame, which was the clean fluorescence of excited atomic iron states (Hartman and Winn, 1978). The metal atoms were produced in a single step by the process

$$
\mathrm{Ar}{ }^{\star}+\mathrm{Fe}(\mathrm{CO})_{5}+\mathrm{Ar}+\mathrm{Fe} e^{\star}+5 \mathrm{CO}
$$

No mulecular or co emission was detected. The bimolecular nature was established by comparing the pressure dependence of the metal fluorescence to the pressure dependence of nitrogen fluorescence produced in a process known to be bimolecular with $\mathrm{Ar}^{*}$. The bimolecular nature has since been confirmed by molecular beam studiez (snyder et al.. 1980; 
Krenos and Kobovitch, 1981).

Subsequently, similar metal atom fluorescence has been seen with both iron and nickel carbonyl from collisions from metastable argon, neon, and helium (Hartman, Hollingsworth, and Winn, 1980). All spin states of the metal were observed. The data was fit to a statistical model, about which more will be said in Chapter IV, which suggested either a concerted, or stepwise, but very fast co ligand loss occurring before molecular rearrangement could occur. This conclusion is based on the data fitting best to the case where no rotation occurred for the carbonyl molecules which is characteristic of a concerted loss of all the co's. In addition to this work, this unique pathway to metal atoms and ions, as well as to the more expected carbonyl fragments, has been observed from iron and some other carbonyls, from methods including multiphoton excitation (Engelking, 1980; Duncan et al., 1979; Karny et al., 1978), vacuum ultraviolet photon photolysis in a bulb (Hellner et al., 1979, 1981) or in a beam (Horak, 1982; Horak and Winn, to be published), and recent work in our laboratory has shown that electron impact also yielas iron fluoresctince. 1

These different methods produce different spin states with only our collisional excitation apparently producing all possible multiplicities of iron, ranging from the singlet through the septet levels. Multiphoton excitation yields only triplets and quintets, whereas single vuv ara

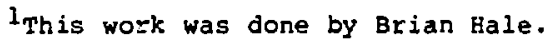


prelimi:ary results from the electron impact work show that only the quintet states are produced. As of yet, there is no satisfactory theory for differences in the dissociation mechanisms which explains the different combinations of spin productions.

In the current work, more work into the nature of the $\mathrm{Ar}^{*}+\mathrm{Fe}(\mathrm{CO})_{5}$ reaction is presented and more analysis has been done for some of the systems previously studied. In addition, new spectra have been obtained with some other carbonyls. 
<smiles></smiles>

$\mathrm{M}\left(\mathrm{COl}_{4} \cdot \mathrm{H} \cdot \mathrm{Ni}\right.$

T。<smiles></smiles>

$M\left(\mathrm{CO}_{\mathrm{g}}, \mathrm{M}=\mathrm{Fe}, \mathrm{Pu}, \mathrm{OS}\right.$

D 3 h

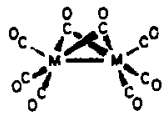

$\mathrm{m}_{2}(\mathrm{CO}), \mathrm{M}=\mathrm{Co}$

$\mathrm{D}_{30}$

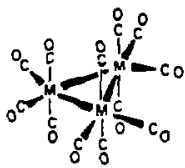

$m_{3}(C O)_{12}, M+R u, O s$

on

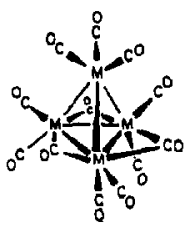

$\mathrm{H}_{4}(\mathrm{CO})_{12}, \mathrm{M}=\mathrm{CO}, \mathrm{Rh}$

$c_{3 n}$

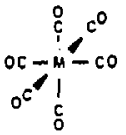

$M\left(\mathrm{CO}_{G}, H=Y, C r, M o, w\right.$

$\sigma_{n}$

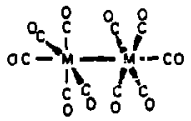

$M_{2}(C O)_{10} \cdot M \cdot M n$.TE.Re

Dad

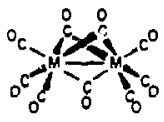

$\mathrm{M}_{2}(\mathrm{CO})_{9}, \mathrm{MAFe}$

$D_{3 n}$

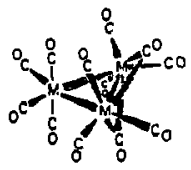

$\mathrm{M}_{3}\left(\mathrm{CO}_{12} \cdot \mathrm{MaFe}\right.$

$\mathrm{C}_{2}$ v

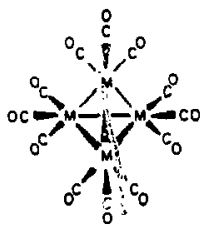

$M_{4}(\mathrm{CO})_{12} \cdot M=I r$

T.

Figure 1-1. Geometries of some smaller metal carbonyls. 
Chapter II

Experimental

As discussed in Chapter $I$, the basic experimental method employed was that of a flowing afterglow apparatus, as modeled after setser's basic design (stedman and Setser, 1972). A schematic view of the apparatus is given in Figure 2-1. The major part of the apparatus consisted of a stainless steel chamber with ports arranged on all four sides and the top as well. A chamber containing a resistively-heated oven was situated directly below for use with reagents having low vapor pressures at room temperature; the oven was not used for these studies and was closed off. A Weich mechanical pump Model 1375 pumped the whole apparatus. Its pumping speed of 1000 liters per minute translated into a measured gas flow of $7.3 \times 10^{3} \mathrm{~cm} \mathrm{sec}^{-1}$ at: 1 torr of argon.

The ports could assume various functions. The one facing the monochromator was fitted with a window in order to feed the reaction chemiluminescence to the monochromator entrance slit. The upstream port admitted the discharge tube into the chamber with a cajon-coupling connection. The downstream port coupled to a foreline leading to the pump. The top port and the remaining side port lopposite the window to the monochromator) at various times admitted reagent(s), introduced lamp radiation through a window for absorption experiments, or were blanked off when not in use.

The system pressure was monitored by an MKS Baratron type 170M-6B cafacilance manometer attached tos the pump 
foreline. Its output of 0-10 volts d.c. gave directly relative pressures in the range of 0.0001 to 1 torr for 0.1 to 1000 microns). The reference side of the pressure head was pumped continuously with a small welch mechanical pump Model 1400. With this arrangement, background variation of the zero pressure value was guite smal:.

The experiment consisted of the following basic steps:
A) Production of the metastable state.
B) Introduction of reagent(s).
C) Observation of product fluorescence.
D) Data storage and manipulation.

Each part will be discussed separately beginning with:

A. Metastable production.

The rare gases were available through campus supplies with high purity. Some background gases, such as $\mathrm{H}_{2} \mathrm{O}$, were removed by passing the flow through a liquid nitrogen-cooled trap. Molecular sieve was at various times used in the trap, but the concentration of the metastable state was relatively unaffected by its use. A titanium oven was briefly inserted in the Iine before the trap to remove $\mathrm{N}_{2}$ by adsorption, but the constriction it caused in the flow rate made it impractical.

The gas was excited in a d.c. electric discharge of around 275 volts with the negative electrode lying upstream. Metastable production was relatively insensitive to voltage change in this range. It was more sensitive 
however to the resistance maintained with a potentiometer in series with the d.s. power supply; the resistance was set to maximize the metastable concentration, and resulted in currents on the order of 1 milliamp.

Making discharge tubes remains, at least for me, a very qualitative science, and somewhat akin to building a ship in a bottle. Glass of 0.75 inches $0 . d$. was used. As discussed by kolts and Setser (1978), diffusion of the metastable state to the walls, where metastable quenching is very efficient, is governed by the diameter of the tube. The larger the diameter of the tube is, the less is the concentration loss. However, an attendantly greater pumping speed would be required. Tubes of 0.75 inches $0 . d$. worked well with argon; since helium and neon are lighter, and thus faster diffusers, this size tube was less efficient for their metastable productions.

Holes were blown in the glass tube through which electrodes were fed and epoxied. The electrode material was 0.004 inch tantalum foil spot-welded to steel wire. Various electrode shapes were tried with the idea being to maximize the volume for which the electric field would be high without greatly increasing gas turbulence. The design which worked best had both electrodes being $2.5 \mathrm{~cm}$ long cylinders, roughly $1 \mathrm{~cm}$ in diameter and $1 \mathrm{~cm}$ apart at closest points.

For argon, the discharge was sustainable from about 0.15 tory on up to past 3 torr. Most of the work was done at 1 torr of pressure where the metastable concentration was 
highest. The relative concentrations of metastable argon as a function of total argon pressure are shown in Figure 22. The absolute metastable concentration was measured using atomic absorption and the results are discussed in chapter III.

B. Introduction of reagent(s).

In most cases, the reagent was introduced through the port directly opposite the window to the monochromator so that all product fluorescence would be detected by the monochromator. The capability existed though to introduce the reagent further upstream so that only unexeited products would be present by the time the flow reached the viewing region. The fluorescence took the form of a hollow cone, corresponding to the mixing interaction between the incoming stream of argon and the carbonyl from the side. In the extreme of highest pressure of the carbonyl (about 20 to 30 microns) ard low argon pressure, the flame was sinort and sharply defined. In the other extreme of very low carbonyl pressure (less than 1 micron), the flame was more diffuse, appearing as a broad band with no end visible.

Liquids such as $\mathrm{Ni}(\mathrm{CO})_{4}$ and $\mathrm{Fe}(\mathrm{CO})_{5}$ were contained in a metal cylinder connected to the chamber through a valve and 0.25 inch steel. tubing. Both of these carbonyls were so volatile that constriction was necessary to result in a sufficiently low pressure. However, due to the much lower vapor pressures of the solid carbonyls, similar arrangements 
were not successful in producing sufficiently high pressures for them. Attempts at heating the cylinder slightly by wrapping it in heating tape resulted in clogging due to deposition of the carbonyl in the valve where less efficient heating and greater constriction occurred. Using the oven below was not successful since the large volume of the oven chamber caused the small amount of the carbonyl to be too widely dispersed.

Finally, good results were obtained by designing a glass tube of 0.5 inches $0 . d$. Which was fed directly into the chamber via a cajon-coupling connection. Without a valve in the line and with the wider tubing, the sample could be more efficiently pumped and, in addition, heating was easier. Sufficient pressure in most cases resulted with only gentle heating from a variac-controlled heating tape wrapped around the glass tube. But without a valve in the line, there was little control over the pressure admitted into the chamber, other than by increasing or decreasing the amount of heating.

Pressure values were read using the baratron. For the pressure studies of Chapters III and $V$, accurate values of both the carbonyl and argon pressures were necessary. However, there was roughly a factor of 100 to 1000 difference between the two pressures. This made direct combined pressure monitoring of both pressures impossible except for the higher pressures of the carbonyl. The only recourse was to measure the carbonyl flow separately. It 
was not a good assumption though that this would be the pressure of the carbonyl when added on to a flow of argon 1000 times greater. The real pressure would be lower since the carbonyl would be trying to effuse into a viscous flow of argon. In addition, the effective pumping speed of the pump would be less with a large flow of argon present. It was necessary then to make measurements of the carbony 1 with and without argon present for larger pressures where the combined measurement was possible. This calibration was used to extripolatc to the lower pressures of carbonyl measured directly with no argon present.

Some common background gases are unfortunately efficient quenchers of the metastable gases used, due to the resonant energy transfer between the metastable and at least one energy level of the gas. The most troublesome for Ar was transfer to the $\mathrm{N}_{2}\left(\mathrm{C}^{3} \Pi_{\mathrm{u}}\right)$ level which resulted in the $\mathrm{N}_{2}$ $\left(C^{3} \pi_{\mathrm{U}} \rightarrow \mathrm{B}^{3} \Pi_{\mathrm{g}}\right)$ transition which gave strong band heads at 337 $\mathrm{nm}, 358 \mathrm{~nm}$, and $381 \mathrm{~nm}$. In addition, background $\mathrm{H}_{2} \mathrm{O}$ would lead to the $\mathrm{OH}\left(\mathrm{A}^{2} \Sigma^{+}+\mathrm{X}^{2} \Pi_{i}\right)$ band appearing strongest $\exists$ round 300 to $315 \mathrm{~nm}$. For helium, the $\mathrm{N}_{2}{ }^{+}\left(\mathrm{B}^{2} \Sigma_{\mathrm{u}}^{+}+\mathrm{X}^{2} \Sigma_{\mathrm{g}}^{+}\right.$) was a very strong band system appearing strongest at $392 \mathrm{~nm}$.

The problem with these band systems was that first, it was impossible to eliminate all of the nitrogen and the water, and second, the quenching resulted in the lessening of the metastable concentration. Most importantly though, the rather wide bands were in the region where atomic metal lines frequently appear, thus making identification of these 
states hard and intensity assignment difficult for the lines. that could be assigned. The only good aspect of these background gases was that there was always a guick way to $\cdots$ monitor relative metastable concentrations through band strength. Also, it was a good leak-jetecting method; that is, all one had to do was just triddle and tighten the various fittings while watching for a reduction in the $\mathrm{N}_{2}$ band intensity.

C. Observation of product fluorescence.

The chemiluminescence from the reaction zone was fed thro:gh a quartz window into a Jobin-Yvon 1.5 meter grating monochromator, capable of doubie pass operation. The enirance and exit slits were continuously adjustable up to 1500 microns $(1.5 \mathrm{~mm})$. Typical experimental conditions of 1000 micron slits and single-pass operation gave a resolution of about 2 A $(0.2 \mathrm{~nm})$. An RCA C31034 photomultiplier tube cooled to ary ice temperature was used to detect the signal. The wiring diagram used for the photomultiplier tube is shown in Figure 2-3. At dry ice temperature, the dark count was 3 counts per second. Tube response was good from 2000 to 8000 A. Hartman (1979) conducted a calibration of the relative response of the photomultiplier tube versus wavelength with a tungsten-filament incandescent lamp by comparing experimental intensities to calculated intensities of a black-body raciator having the same temperature as the lamp. The relative response 
curve is shown in Figure 2-4. Experimental intensities were always normalized against this curve to eliminate PMT effects. For wavelengths below 3500 A where the calibration was no: performed, the approximate relative response was obtained by extrapolating from the end of the curve in Eigure 2-4. Published response curves for this tube indicate that the extrapolation used was in reasonable agreement with the true tube response. The output from the PMT was discriminated and fed into a Nicolet 1310 signal Averager.

The two basic experiments consisted of either scanning the monochromator versus wavelength and storing the channels in the Nicolet, which had 4096 channels available, or averaging the intensity at a particular wavelength. For data averaging, the Nicolet had excellent capabilities for integrating and thoroughly manipulating the data. Thus, it was easiest to work up the data in the Nicolet and simply write down the averaged value.

For the scanning experiment, the usual scanning speed was 50 A per minute with a 1 second per channel collection rate, corresponding to 0.83 per channel. This signal was output from the Nicolet in RS232C serial peripheral form of a sequential train of characters. The signal went at a baud rate of 300 characters per second to a TNW RS232C/IEEE-488 converter controlled by a commodore PET $32 \mathrm{~K}$ microcomputer; this slow baud zate was necessitated by the relatively slow way in which BASIC works. The final IEEE format was 
compatible with and fed into the PET for eventual storage and manipulation.

An alternate detection system used at various tines t.. comprised of a Jarrell-Ash 0.25 meter monochromator, model 82-410, attached to a Tracor Northern Ts-1710 DARSS optical multi-channel analyzer system with an intensified detector head having a diode array of 1024 channels. With the Jarrell-Ash, approximately 600 A could be simultaneously monitored. This arrangement lent itself to data averaging experiments where a series of closely-spaced peaks could be monitored under precisely the same experimental conditions. However, the big problem with this set-up was the low sensitivity of the DARSS when compared to the PMT. The sensitivity was especially poor below 4000 , which unfortunately was the area where many of the metal 1 ines of interest were located. This poor sensitivity was in spite of the fact that the detector head was intensified and coated with a scintillator to extend its response further into the near UV.

D. Data storage and manipulation.

A series of grograms was created to facilitate the handing of the data coming from the scanning experiments. They are reproduced in Appendix I. Here, a brief description of each program is given. The specifics are also in the appendix. Chapter IV deals with the processes they concern in more detail. 
1) "LINE DATA". (not listed in the Appendix) This program helped make a little less tedious the compilation of the lines from the strongest transitions arising from the various energy levels of different metal atoms. It calculated state lifetimes, printed out the strongest lines arranged by energy level or by wavelength, and stored the data file on a PET disk for later use.

2) "NICOLET $\rightarrow$ PET" . The program first controlled data acquisition from the TNW interface and the Nicolet. It then stored the data on disk.

3) "DATA PROCESS". This program, the nerve center of the whole operation, first read in the channel data from the disk, found the limits of intensities, scaled the data, and formatted a plot to the Hewlett Packard 7245A Plotter printer, giving the wavelength plots that are seen later in Chapter VI; it had replotting and rescaling capabilities. Then it found all peaks in the data over a specified cutoff value, printed and stored them. It could correct for the PMT efficiency (Figure 2-4), could least squares fit the strongest $l$ ines to known wavelengths, and could accept real values of wavelengths. An assigned, intensity-corrected set of peaks was the result and was stored on the disk.

4) "P \& R PLOTTER". This program took the corrected and assigned line data from the disk or from the user, loaded the requested metal line file as compiled by LINE DATA and calculated the steady-state populations and relative formation rates for the various energy states of the metal; 
for details, see chapters IV and VI. For the population Flot, it could perform a least squares fit for all or part (if the data, draw the best line and print the temperature to which the distribution corresponded. For the rate plot, all or part of the data could be fit with either linear least squares analysis to determine the power dependence, or fit using a non-linear least squares method to determine the best value for the available energy; the least squares curve could then be plotted on the graph. The population and rate data were stored on the disk. 
Eigure 2-I. The flowing afterglow apparatus. 


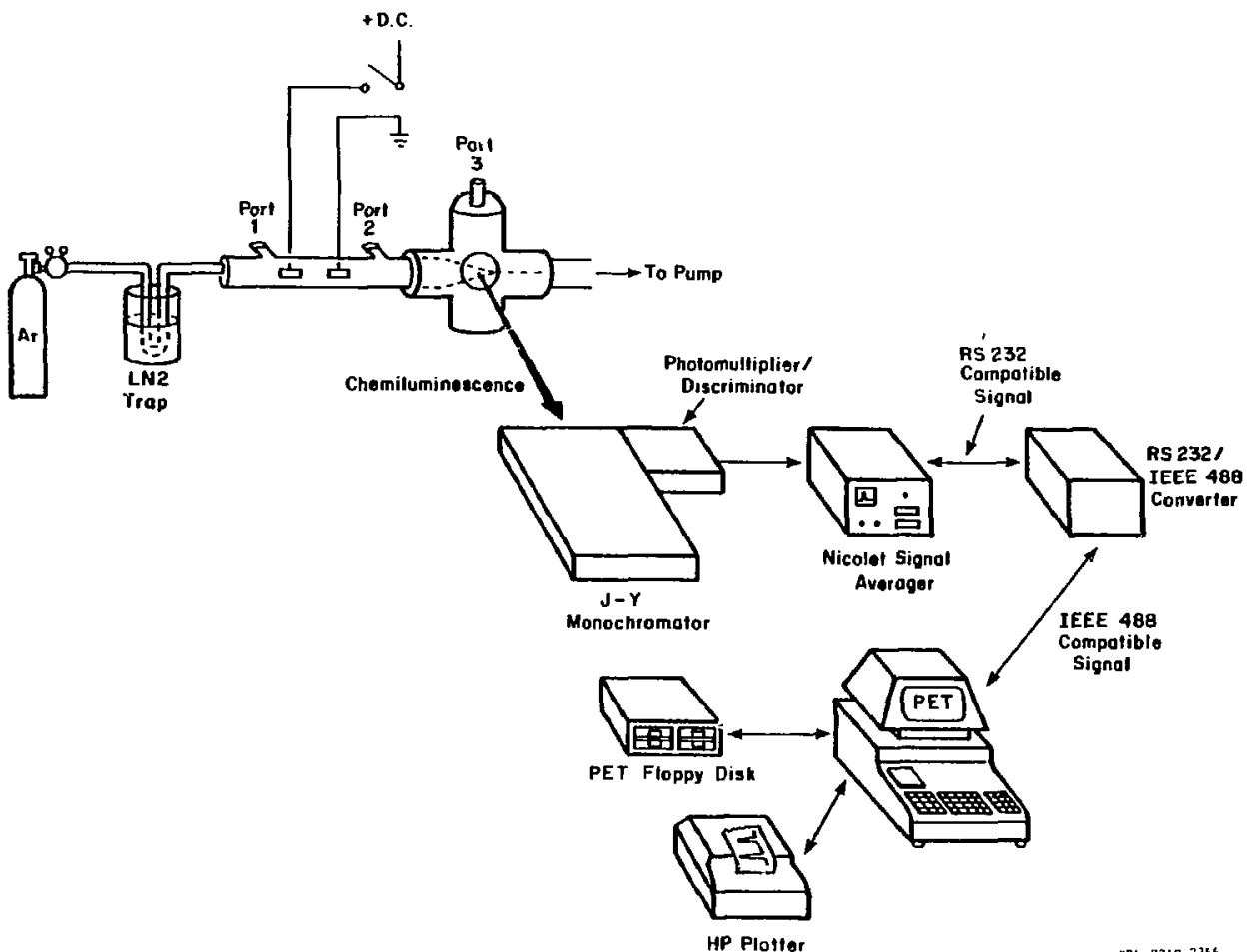




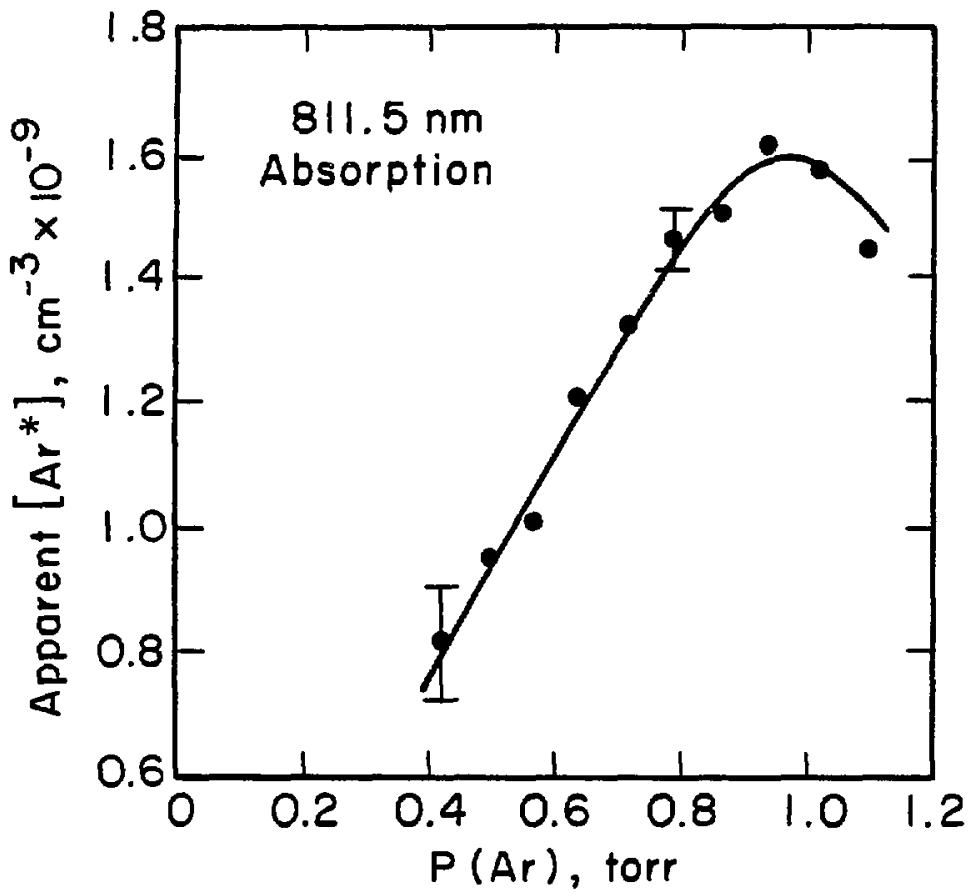

XBL $7910-12390$

Figure 2-2. The concentration of the argon ${ }^{3} p_{2}$ metastable state as a function of argon pressure. 
Figure 2-3. The dynode chain wiring used for the PMT C31034. 


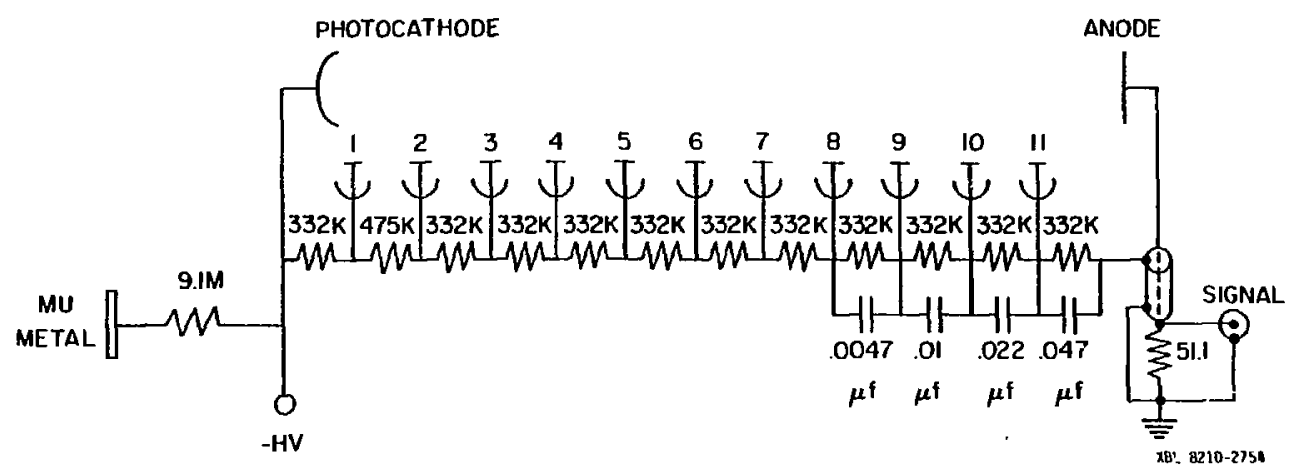

RCA $C 31034$ 
Figure 2-4. Relative response of the photomultiplier tube. 


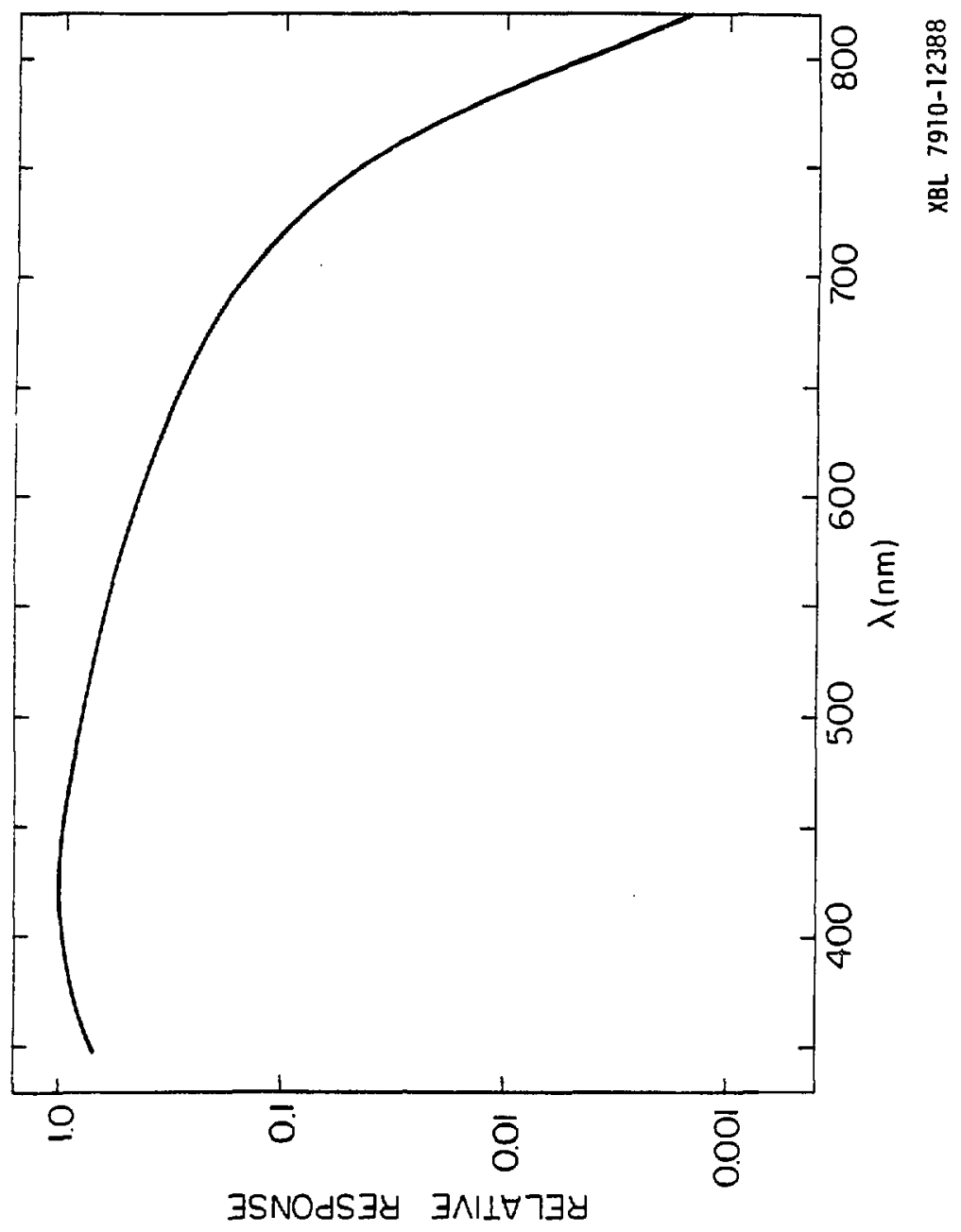


Chapter III

Absorption Experiments

Atomic absorption was used to determine severzl things. First, metastable concentrations for both of the metastable states of argon were determined. Second, the total bimolecular rate constant for the process $\operatorname{Ar}^{\star}\left({ }^{3} P_{2}\right)+$ $\mathrm{Fe}(\mathrm{CO})_{5}$ was determined through the monitoring of the signal absorption of an argon line emanating from this state for a series of pressures of $\mathrm{Fe}(\mathrm{CO})_{5}$ and argon, and then comparing to a series of pressures for the reaction of argon with krypton since the rate constant for the reaction $A r^{\star}\left({ }^{3} \mathrm{P}_{2}\right)+$ $\mathrm{Kr}$ is known (Gundel et al., 1976).

An oriel Ar spectral lamp provided a strong source of argon 1 ines. The experiment to determine the ${ }^{3} \mathrm{P}_{2}$ metastable concentration had been done before, but with all the lamp radiation impinging upon the gas flow while absorption of only one particular line was monitored. This method could not cule out the possibility of other lines inducing processes which could affect the metastable state population. This time, a Jarrell-Ash monochromator was inserted between the lamp and the chamber; by acting like a narrow bandpass Eilter, the monochromator ensured that the line being monitored was also the only one interacting.

A. Metastable concentrations of argon ${ }^{3} \mathrm{P}_{2}$ and ${ }^{3} \mathrm{P}_{0}$.

Atomic absorption can give a good estimate of metastable consentration by measuring the degree of attenuation 
of a line arising from the state as it passes through the metastable flow using the basic Beer's law relation:

$$
\ln \left(\frac{I}{I_{0}}\right)=-k_{i k} \times \ell \times\left[A r^{*}\right]
$$

where $I_{0}$ is the unattenuated signal in counts per second, [Ar"] the number density in $\mathrm{Cm}^{-3}$ of the metastable state, $x$ the path length in cm through the flow, and $K_{i k}$ the absorption coefficient for the particular line in $\mathrm{cm}^{2}$.

The general form for the absorption coefficient for a Doppler I ine shape at the line center is (Ivanov, 1973):

$$
R_{i k}=\frac{1}{8 \pi^{1.5}}\left(\frac{m}{2 R \ln 2}\right)^{1 / 2} \frac{g_{k} A_{k i}}{g_{i} v_{i k}}
$$

where $m$ is the gas molecular weight, $v_{i k}$ the transition frequency in $\mathrm{cm}^{-1}, g_{i, k}$ the degeneracies of the lower and upper states, $A_{k i}$ the Einstein coefficient for spontaneous emission in $\sec ^{-1}, \mathrm{R}$ the gas constant, and $\mathrm{T}$ the temperature in degrees Kelvin. Substituting in all the constants at room temperature for a gas with the mass of argon yielos:

$$
k_{i k}=7.65 \times 10^{-7} \frac{g_{k} A_{k i}}{g_{i} v_{i k}^{3}}
$$

This Doppler line shape is a good representation of the true line shape as long as the Doppler width $\left(\sim 7 \times 10^{8}\right.$ sec $\left.^{-1}\right)$ is large compared to the sum of the collisional linewidth (small as measured for this lamp pressure) and the natural line width, which is approximately $A_{k i} / 4 \pi$. This indicates 
that the assumption is valid up to an Einstein coefficient of about $10^{9}$, and allows for all of the transitions of argon.

The Ar 8115.3 A line was chosen to measure the ${ }^{3} \mathrm{P}_{2}$ concentration. For this transition $(93144-105463), g_{i}=$ 5, $g_{k}=7, v_{i k}=12319 \mathrm{~cm}^{-1}$, and $A_{k i}=3.66 \times 10^{7} \mathrm{sec}^{-1}$ (wiese et al., 1969) which gives an absorption rate constant of $k_{i k}=2.10 \times 10^{-11} \mathrm{~cm}^{2}$. The measured attenuation at 1 torr of argon was $I / I_{U}=0.74$, giving a concentration for the metastable state of $2.74 \times 10^{9} \mathrm{~cm}^{-3}$, using an estimated gath length of $5 \mathrm{~cm}$.

To measure the ${ }^{3} \mathrm{P}_{0}$ concentration, the Ar 7948.2 \& line (94554 - 107132), was used. The data on this line are $g_{i}=$ $1, g_{k}=3, v_{i k}=12578 \mathrm{~cm}^{-1}$, and $A_{i k}=1.96 \times 10^{7} \mathrm{sec}^{-1}$. This gives a rate constant of $k_{i k}=2.26 \times 10^{-11} \mathrm{~cm}^{2}$. With the measured attenuation of $I / I_{0}=0.95$, the resulting concentration was $4.54 \times 10^{8} \mathrm{~cm}^{-3}$.

As discussed in Chapter $I$, the ${ }^{3} \mathrm{P}_{0}$ to ${ }^{3} \mathrm{P}_{2}$ state concentration ratio is predicted to fall even lower than the predicted statistical ratio of 1:5 due to the more efficient quenching to other argon states. Here, it's found to be $1: 6.0$ in relative concentration. So the ${ }^{3} \mathrm{P}_{2}$ metastable state is indeed the major carrier, and represents $86 z$ of the total metastable population.

B. Total rate constant for $\mathrm{Ar}^{\star}\left({ }^{3} \mathrm{P}_{2}\right)+\mathrm{Fe}(\mathrm{CO})_{5}$.

With our flowing afterglow apparatus, we could detect 
only fluorescence, meaning that only the fraction of the total reaction rate which produced excited iron atoms that fluoresce to lower states was observed. Ground state production and molecular ionization were competing channels which we could not monitor, so it was useful to measure the total rate constant and hence the branching ratio for the fluorescent channel.

Hartman (1979) calculated rate constants for the production of the various excited states. This was done by comparing $\mathrm{Fe}$ intensities to the intensities of the $\mathrm{Kr} 7602$ \& line which comes from a state of $\mathrm{Kr}$ whose production rate from the reaction of $A x^{*}\left({ }^{3} \mathrm{P}_{2}\right)$ with $\operatorname{Kr}\left({ }^{1} \mathrm{~S}_{0}\right)$ is known to be $6.2 \times 10^{-12} \mathrm{~cm}^{3} \mathrm{molec}^{-1} \mathrm{sec}^{-1}$ (Gundel et al., 1976). For the lowest observed multiplets of $\mathrm{Fe}$, this gave rate constants in the range of $5.0 \times 10^{-13}$ to $5.0 \times 10^{-14} \mathrm{~cm}^{3}$ molec $\sec ^{-1}$, yielding an estimated total fluorescent rate constant of $6 \times 10^{-12} \mathrm{~cm}^{3}$ molec-1 $\mathrm{sec}^{-1}$ (Hartman et al., 1980). For the collision pair of argon and iron carbonyl at $300 \mathrm{~K}$, the rate constant $\mathrm{k}$ and the thermal cross-section $\langle\langle\sigma\rangle$ are related by

$$
\left\langle\langle\sigma\rangle=2.29 \times 10^{11} \mathrm{k}\right.
$$

where $\langle\langle\sigma\rangle\rangle$ is in $A^{2}$. This gives an estimated total fluorescent cross-section of $\sim 1 A^{2}$. Note that this doesn't include the ground state metal production. The total ionization cross-section for the same bimolecular process using a crossed molecular beam apparatus with an average 
collision energy of about $190 \mathrm{meV}$ was measured to be $\sim 20 \mathrm{~A}^{2}$ (Snyder et al., 1980). This energy corresponds to a temperature much higher than room temperature; the average collision energy at room temperature for this process would be about $40 \mathrm{mev}$.

In the current experiment, the argon 8115 A line was monitored as described in section $A$. of this chapter. This was done for a series of pressures of argon and iron carbonyl as well as for argon and krypton, and the results are shown in Figures $3-1$ and 3-2. Knowing the total rate constant for $\mathrm{Ar}^{*}\left({ }^{3} \mathrm{P}_{2}\right)+\mathrm{Kr}$, then the rate constant for the quenching by $\mathrm{Fe}(\mathrm{CO})_{5}$ could be obtained, as shown in the following kinetic scheme.

With no quenchers present (other than argon itself) at a given pressure of argon, $A r^{*}$ is produced as some rate $k_{f}$ (units of $\sec ^{-1}$ ) and destroyed by quenching with other argon atoms at a rate of $k_{d}$. Then, the steady state equation that pertains is:

$$
\frac{d\left[A r^{*}\right]}{d t}=0=k_{f}-k_{d}\left[A r^{*}\right]
$$

or,

$$
\left[A E^{*}\right]=\frac{K_{E}}{k_{d}} .
$$

Upon the addition of a given pressure of $\mathrm{Xr}$ (or $\mathrm{Fe}(\mathrm{CO})_{5}$ ) to the system, an additional quenching channel is introduced and the metastable concentration is further reduced:

$$
\frac{d\left[A r^{*}\right]}{d t}=0=k_{f}-\left[A r^{*}\right]\left(k_{d}+k_{K r}[R r]\right)
$$


or,

$$
\begin{aligned}
{\left[A r^{*}\right] } & =\frac{k_{f}}{k_{d}+k_{K r}[K r]} \\
& =\frac{k_{f} / k_{d}}{1+\frac{k_{K r}[K r]}{k_{d}}} .
\end{aligned}
$$

The only unknown is $k_{d}$. Once this is determined using data from the krypton experiment, the analogous equation for $\mathrm{Fe}(\mathrm{CO})_{5}$ can be solved to determine $\mathrm{k}_{\mathrm{Fe}(\mathrm{CO})_{5}}$. Drawing upon eqs. 3.1 and 3.5, the equation solved for $k_{d}$ in terms of experimental observables is:

$$
k_{\mathrm{d}}=\frac{k_{\mathrm{Kr}}[\mathrm{Kr}]}{\left(\frac{\ln \left(\mathrm{I}_{\mathrm{Ar}} / \mathrm{I}_{0}\right)}{\ln \left(\mathrm{I}_{\mathrm{AI}+\mathrm{Kr}} / \mathrm{I}_{0}\right)}-1\right)}
$$

where $I_{A r}$ is the intensity of the 8115 line with just the metastable flow of argon, and $I_{A r+K r}$ the intensity with krypton added to the flow. With $k_{d}$ determined, the final expression for the total rate constant of $\mathrm{Fe}(\mathrm{CO})_{5}$ is:

$$
k_{F e(C O)_{5}}=\left(\frac{\ln \left(I_{A r^{\prime}} / I_{0}\right)}{\ln \left(I_{A r+F e}(C O)_{5} / I_{0}\right)}-1\right) \frac{k_{d}}{\left[F e(C O)_{5}\right]} .
$$

To eliminate possible effects of flame shape upon the amount of absorption of the signal, the rate constants were determined at similar pressures of $\mathrm{Fe}(\mathrm{CO})_{5}$ and $\mathrm{Kr}$. Taking the average of the rate constant at several different pressures gave $\mathrm{k}_{\mathrm{Fe}(\mathrm{CO})_{5}} \sim 1.6 \times 10^{-11} \mathrm{~cm}^{3} \mathrm{molec}^{-1} \mathrm{sec}^{-1}$, 
which corresponds to a cross-section of $\sim 4 A^{2}$. The error in this value is hard to estimate but it could, potentially, be significant. It depends on how well $\mathrm{k}_{\mathrm{Kr}}$ is known, on two pressure calibrations for $\mathrm{Fe}(\mathrm{CO})_{5}$ and $\mathrm{Kr}$ flows, and on effects of background gases upon total argon absorption.

These values indicate roughly a $1: 3$ fluorescent to dark channel branching. The value of $20 A^{2}$ for the ionization channel (Snyder et al., 1980) is significantly higher than the value here $\left(<3 \mathrm{~A}^{2}\right)$. However, their work was performed at a collisional energy greatly higher than ours. Initial estimates indicate though that the ionization cross-section increases with decreasing collisional energy, making the room temperature ionization channel even more dominant. However, in a paper dealing with a series of room temperature chemi-ionizing reactions from collisions of metastable argon, no molecule was found where the ionization channel was the dominant one (Golde et al., 1982). This matter still needs to be resolved. 


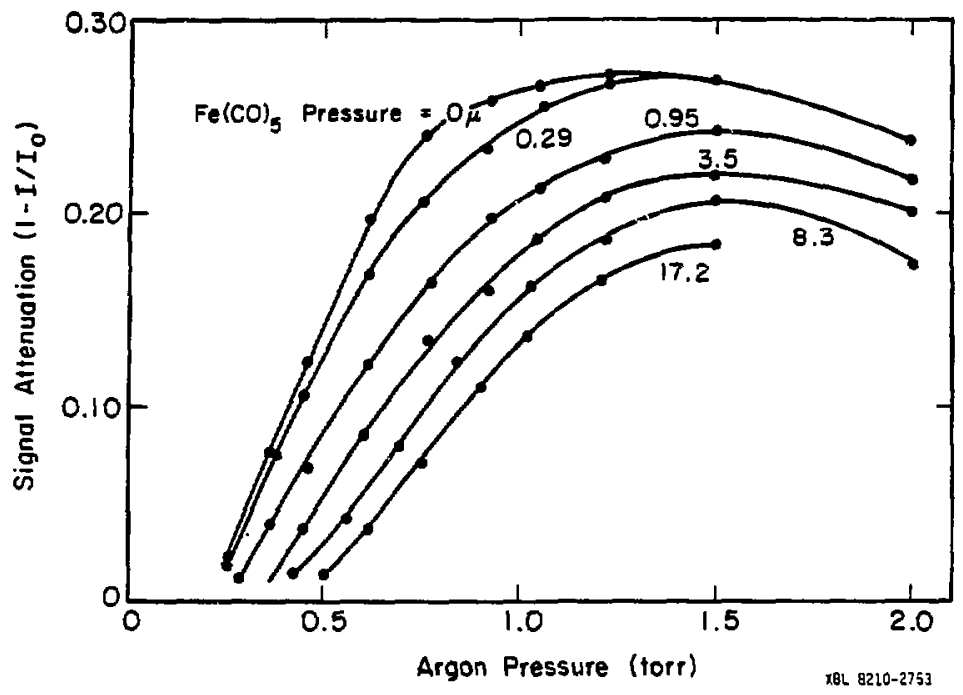

Eigure 3-1. Attenuation of the $8115 \mathrm{~A} A \mathrm{Ar}$ line as a function of argon pressure at different pressures of iron carbonyl. 


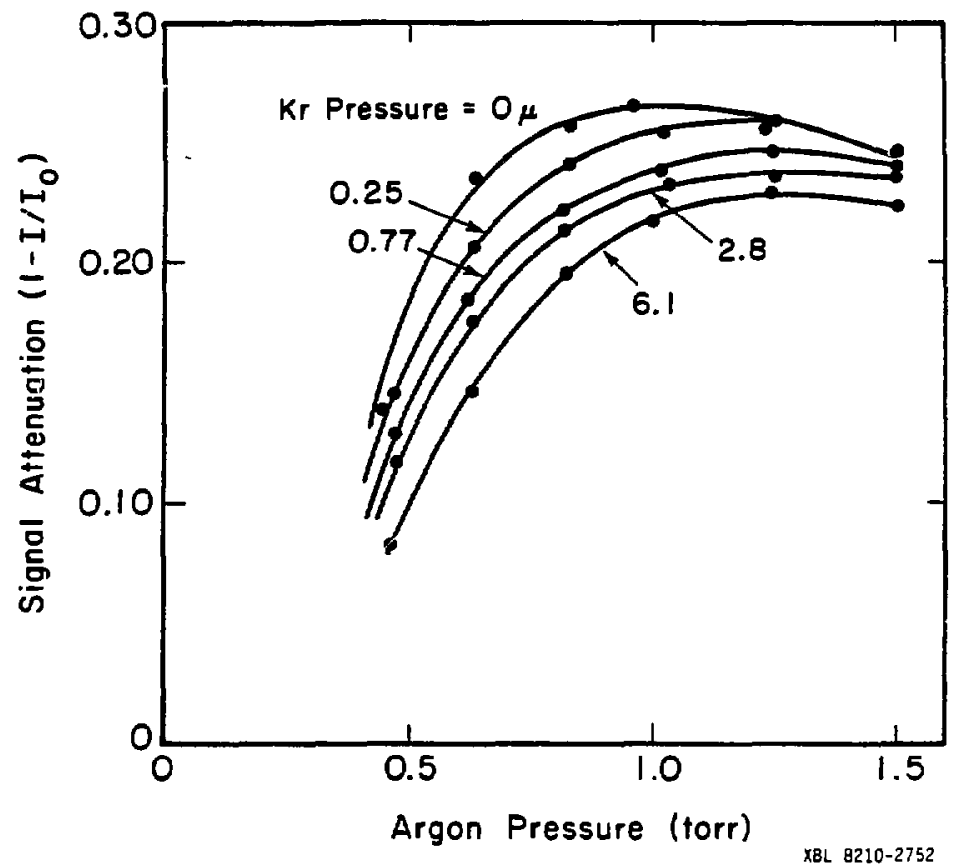

Figure 3-2. Attenuation of the 8115 A Ar line as a function of argon pressure at different pressures of krypton. 
Chapter IV

What Metal Transition Intensities Tell You

It's time to consider in more detail the main experimental observables that come from the steady-state flow experiments, namely the metal line intensities arising from the unique bimolecular production of metal atom states from theic collisional excitation with metastable rare gas states. In this chapter, the ideas will be developed and tested on $\mathrm{Fe}(\mathrm{CO})_{5}$ and $\mathrm{Ni}(\mathrm{CO})_{4}$; newer resuics from other carbonyls will be presented in the next chapter.

First, and most obviously, some information is obtainss from a simple energy accounting. The initial energy injutis the specific metastable energy of the rare gas, $\mathrm{E}^{*}$. To get to a system of metal atom', all of the metal-carbonyl bonds must be broken, requiring a total energy of $E_{b}$, the so-called energy of disruption in inorganic jargon. The remaining energy can go into the production of the metal excited states. This "available" energy, $E_{a}$, can be expressed

$$
E_{a}=E^{\star}-E_{b}
$$

$E_{a}$ is the maximum energy that can go into the metal atom. From observing the highest energy level of the metal formed, the experiment gives an upper limit to the total bond energy of the carbonyl. For most carbonyls, this value is not very well known and an experimental verification of values 
derived from thermodynamic calculations and assumptions would be useful.

Several reasons exist as to why only an upper limit to the total energy is possible. In the first place, some of the energy may have gone into other modes such as translation of the metal atom, and translation, vibration, or rotation of the co molecules. Secondly, there is by no means a continuum of metal states available; instead, there often is a gap of several 1000 wavenumbers or more between states. Thirdly, not all states have transitions in the range we are monitoring. And finally, states near the thermodynamic limit of energy are generally produced at very small rates, and thus would escape detection. Ours is not an absolute detestion system, and even if these states were present, with their very small intensities they would be very hard to ascertain, and would not be distinguishable from background signal. For more discussion on relative rates of formation, see section $B$. of this chapter.

Intensity (in counts per second) is the rate of emission from a state and is dependent upon both the population of the metal state, $\left[M^{*}\right]$, and the rate at which the state can fluoresce to lower states, namely the Eirstein A coefficlent. For a steady-state system, the rate of formation $\mathrm{K}_{f}$ of a particular state is balanced by the ste of loss of all the lines from that state. The rate of change in population is

$$
\frac{d\left[M^{*}\right]}{d t}=0=k_{f}-\sum_{i} I_{1 U}
$$




$$
\frac{d\left[M^{\star}\right]}{d t}=K_{E}-A_{u}\left[M^{*}\right]
$$

where $A_{u}$, the Einstein coefficient for the state as a whole, is equal to the sum of all the A's for the particular transitions for the state, reflecting the relative probabilities of these transitions.

The rate at which the steady-state population for a state is reached is obtained by solving the differential equation 4.3. With the boundary condition that the initial population is zero the solution is:

$$
\left[M^{\star}(t)\right]=\frac{R_{f}}{\bar{A}_{u}}\left(1-e^{-A_{u} t}\right) .
$$

The time required to establish equilibrium is seen to be totally dependent upon the transition probability. After a time of three times the lifetime of the state has passed, $3 / A_{u}$, the population has reached 958 of its steady-state value, For even the longest-lived states, with $A_{U} \sim 10^{4}$, the steady-state value is reached in milliseconds.

A. Population and Rate Plots.

From the discussion above, there is now enough information to construct plots of the relative populations and formation rates of the excited states of metals produced in the bimcitcular process. The resulting plots for nickel and iron carbonyl springing from collisions with the rare gases Ar, Ne, and He have appeared (Hartman, 1979; Hartman et al., 1980). The data have been zeanalyzed here using a refined 
process and with a slightly different interpretation; the resulting plots appear later in this chapter.

The major difference was in the interpretation of what was actually being plotted. The observed intensities result from the rate of formation of a manifold of $g$ degenerate states, where $g=2 \mathrm{~J}+1$. Although it is valid to consider the rate of formation as simply this intensity, this gives the rate of formation for : set of states. More fundamentally, the interest is in the production of a jiven state, so the more proper thing to consider as the correct formation rate is $\mathrm{I} / \mathrm{g}$. Before this was done, the states with the highest $J$ values were appearing highest in rate. Once the states were nomalized to their $J$ values, the spread in the data was reduced substantially, permitting more precise curve fitting.

The intensities essentially give you the rate of formation, but it's the summed intensity from all the transitions together emanating from the one energy level which gives the formation rate. Thus, the one transition obtained experimentally had to be corrected by a branching ratio to account for all the other transitions that are occurring. The correct rate then is:

$$
x_{f}=\left(\frac{I}{g_{u}}\right) \frac{A_{u}}{A_{i u}}
$$

where $A_{i u}$ is the transition probability of the observed transition and $A_{U}$, the sum of all the component $A_{i u}$ 's for the state, is the transition probability for the whole 
state.

To obtain a population flot, all that is needed is a slight reworking of egs. 4.2 and 4.3 . Since $\left[M^{*}\right]=I_{i U} / A_{i L}$, the correct form of the population plot is

$$
\left[M^{*}\right] \propto\left(\frac{I_{i u}}{g_{u}}\right) \frac{1}{A_{i u}} .
$$

Since transition probabilities are often listed in data tables in terms of oscillator strengths instead of A coefficients, it was more useful to develop equations in terms of oscillator strengths. The relationship between $A_{i u}$ and the oscillator strength $f_{i u}$ is (Mavrodineanu and Boiteux, 1965):

$$
A_{i u}=6.67 \times 10^{15} \frac{f_{i u}}{\lambda^{2}}
$$

where $\lambda$, the wavelength of the line, is in $A$. So the expression for the relative population used was

$$
\left[M^{*}\right] \propto \frac{I_{i u} \lambda^{2}}{g_{u} f_{i u}} .
$$

For Maxwell-Boltzmann distributions, the temperature is a useful concept and is introduced in the definition

$$
\left[M^{*}\right]=g_{u} \exp \left(\frac{-E_{u}}{k T}\right)
$$

or,

$$
\log \left(\frac{I \lambda^{2}}{g_{u} E_{i u}}\right) \times \frac{E_{u}}{2.3 k T}
$$

where $E_{u}$ is the energy of the upper state of the 
transition. Since this is the identical formalism to our population plots, we can assign temperatures to the straight lines appearing in our plots. A plot of the logarithm of this population vs. the energy of the upper state gives generally monotonically decreasing curves of the type shown in Figure 4-1.

A great deal of speculation centered on the significance of the lines with different temperatures, as seen in Figure 4-2. The distributions of different temperatures were ascribed to the presence of two different mechanisms active in different energy ranges. However, this 1 ine of reasoning was on the wrong track and in reality, the information shown in such a population plot is less informative as to formation mechanisms than the rate plot previously described. The reason for this is inherent in the steady-state process, and can be illustrated by considering eq. 4.4. The steady-state limit for the population is simply $K_{f} / A$. The population then is just the rate of formation modulated by the transition probability for the state. The population of the state is being increased by a non-changing formation rate; assuming that no collisional deactivation is present, the only way that the population can be depleted is through spontaneous emission. But for a very improbable transition, the chances of this occurring are very small for a small population, and the loss is slow in the beginning. Meanwhile, the population continues to build. This continues until the 
population is high enough that the rate of fluorescence (the intensity of the line) can balance she incoming rate of production which is still due to the same small probability of transition but is now acting upon a much larger pool of moleciles. For a short-lived state, this balance occurs much faster, and the steady-state population is consequently much lower.

That the population plot doesn't give any more insight on the dynamics of the process of producing metal atom states doesn't mean that the plots are not interesting. What it does describe is the final population aistribution that the states of a particular metal ends up having due to the production process. One particularly interesting population distribution is the population inversion that was seen for the dissociation of $M\left(\mathrm{CO}_{6}\right.$; see Chapter VI for the full story. That the lowest states usually appear with $v$ astly higher populations reflects the fact that the lowest metal states are often unable to relax rapidly owing to the very few states below them and hence the lesser chance that a lower state will be strongly connected to them through selection rules. This ends up with the same result as for the rare gases and the basis for the flowing afterglow technique, namely that there are low-lying states of metal atoms in the flow which are metastable and are not detected through any fluorescence methods.

I want to express in graphic terms the description of how tedious the data preparation could be. What was needed 
was the branching ratio for a particular transition. A listing of state $A$ values along with the $A$ values for individual transitions would be precisely what is needed. However, the data are rarely tabulated in such a forim. The usual table is instead a listing by wavelength of all known lines for a particular metal. To get the total list of transitions by energy level required scanning all of these lines in order that $[A$ could be calculated. This process had to be repeated for each energy level; the desired streamlining of this process was the motivation for the program "IINE DATA". For metals such as iron which has thousands of known lines over a myriad of levels, the compilation of the strongest lines for each level was excrutiatingly slow. The problem was most tractable for considering only the lowest energy levels, say up to 40,000 $\mathrm{cm}^{-1}$. But for helium and neon with their larger available energies, one had to go essentially to the dissociation limit of the metai to account for all the observed lines. In no case was a complete compilation attempted. At worst though this neglected only very weak lines and complete listings up to $40,000 \mathrm{~cm}^{-1}$ picked up the majo: lines. However, for the testing of the rate theory presented in section $B$. of this chapter, it was necessary to go as high as possible to get the widest possible energy range.

Einstein A values are not known very precisely in most cases and are often no better than to 10 to 208 . This is currently the limiting factor in accuracy of the experiment 
and results in a scatter of data which is characteristic of population and rate plots. Errors are also introduced if not all the lines arising from an energy level are compiled. However, most metals have been studied in enough detail that the strongest lines should always be accounted for. Generally, more than one line would be observed from a particular energy level. This allowed for some averaging which resulted in more reliable data and also made the data less subject to momentary fluctuations in the flame intensity.

B. The statistical density of states model.

That the rate of formation of metal states seemed to decline monotonically with the energy of the upper state and with ro regard to factors such as state symnetry or spin multiplicity suggested that a rather simple model could be proffered to describe the dissociation process. The hypothesis then was that the relative production rate of a metal state at an energy $E_{\mathrm{u}}$ chove the ground state is the result of a purely statistical process and is proportional to the total density of states present at the remaining energy of $E_{a}-E_{v}$ for the active degrees of freedom available in the collision complex. This potentially includes translation of the metal atom and $c o$ molecules, and rotation and vibration of the $\mathrm{CO}^{\prime} \mathrm{s}$. There were assumed to be no ionization channels open or any electronic excitation of the co molecules. 
This hypothesis already explains the two 1 imits of the observed formation rates. For the production of very lowlying metal states, with the limit being ground state production itself, a lot of energy will remain to be parcelled out among the available degrees of freedom. The density of states is very high for this effectively "high temperature" region and production is favored. In the other extreme of production of a metal state lying very close to the thermodynamic limit of energy available, or $\mathrm{E}_{\mathrm{a}}$, then the amount of energy remaining to be dispensed with will be very small; consequently, since there are relatively few states available at this very "low" temperature, the production of this state will be vanishingly small in comparison to the previous case.

What remains to be adjusted in this flexible model is the number of degrees of freedom for each type of component motion open in the dissociation complex. Fitting the data to possible combinations relating to different kinds of collision processes gives some idea as to the nature of the dissociation. The following model will be developed in general terms, and will be tested with the specific examples of $\mathrm{Fe}(\mathrm{CO})_{5}$ and $\mathrm{Ni}(\mathrm{CO})_{4}$ in section $\mathrm{C}$.

To proceed, a total density of states will be needed, starting from the partition functions describing the separate forms of motion of translation, rotation, and vibration. That we are discussing a density of states rather than a degeneracy of states places us in the semi- 
classical limit of the true guantum mechanical result. This approximation is valid as long as the energy considered is large. In this semi-classical limit, the partition function $Q$ and the density of states $P(E)$ are related by (Forst, 1971):

$$
Q=\int_{0}^{\infty} p(E) e^{-E / k T} d E
$$

Total partition functions are simply the product of component partition functions as long as the component motions may be considered independent. On the other hand, density of states are not simply multiplicative; instead, product density of states are obtained through the "convolution" of component densities:

$$
\rho_{1,2}(E) \times \int_{0}^{E} \rho_{1}(E-x) \rho_{2}(x) d x \text {. }
$$

This suggests that the easiest procedure is to start with component partition functions, obtain the product partition function, and then convert to the product density of states as the final result.

The process is even easier due to the fact that an entire mathematical method has been developed which deals with the manipulation of integrals identical in form to eg. 4.11. The method is the Laplace Transform technique. The Laplace transform of a function $F(t)$ in terms of a parameter $s$ is defined (e.g. Churchill, 1972):

$$
\underline{E}\{F(t)\}=\int_{0}^{\infty} F(t) e^{-s t} d t=f(s) .
$$


Upon defining $s=1 / K T$, reminiscent of a very common definition hailing from statistical mechanics, the two integrals 4.11 and 4.13 become identical in form. Thus the following relationship between the partition finction and the density of states:

$$
\underline{L}\{O(E)\}=Q(s) \text { and } \underline{L}^{-1}\{Q(s)\}=\sigma(E)
$$

The individual partition functions for the various forms of motion are, first for translation:

$$
q_{t}=\frac{(2 \pi m k T)^{-i} \omega_{2}}{h} \times s^{-1 / 2}
$$

where $l$ is the length of the container. Since only a relative rate of formation is being developed, dll multiplicative constants will be ignored with impunity. For the rotation of a linear molecule in one airection:

$$
q_{r}=\frac{8 \pi^{2} I k T}{h^{2}}=s^{-1}
$$

where $I$ is the moment of inertia. Finally, for a diatomic harmonic oscillator in the semi-classical limit,

$$
g_{v}=\frac{k T}{h v} \propto s^{-1}
$$

Later, anharmonicity of co vibration will be included. Therefore, for $t$ degrees of translational freedom active, for $r$ linear molecules rotating in one dimension, and $v$ active harmonic vibrators, the total partition function is:

$$
Q=q_{t} g_{t} g_{v}<\left(s^{-t / 2}\right)\left(s^{-r}\right)\left(s^{-v}\right)
$$




$$
Q \propto s^{-(t / 2+r+v)} \text {. }
$$

One useful Laplace transform is:

$$
\underline{L}^{-1}\left\{s^{-n}\right\}=E^{n-1}
$$

So, for the above harmonic model,

$$
\rho_{T \circ t}(E) \propto E^{(t / 2+r+v-1)}
$$

where it is to be remembered that $E$ is a shorthand notation for $E_{a}-E_{u}$, the energy remaining after a given metal state has been produced.

Now, the anharmonic model will be developed for the general case. For the greatly increased difficulty in form and the only slight modification in result, the above harmonic result is probably the more useful model, especially when there is already a lot of error in the data. However, for completeness, the anharmonic model is included.

The anharmonicity of the CO's is introduced empirically by fitting the observed co vibrational energies (Huber and Herzberg, 1979) using the form:

$$
\rho_{v}(E) \approx e^{m E}
$$

For $\mathrm{Co}$, depending on the range of energy to be fitted, m was between the values of $1.30 \times 10^{-5}$ and $7.94 \times 10^{-6}$. This gives a partition function of: 


$$
\text { g. } * \underline{L}\left\{e^{m E}\right\} \times \frac{1}{(s-m)} \text {. }
$$

There is no easy solution for the total partition function including this vibrational component, so the only recourse is to obtain a composite translational-rotational density of states and then find the total density of states by using convolution, eq. 4.11. So,

$$
\begin{aligned}
& \rho_{t, x}(E) \propto I^{-1}\left\{\left(s^{-t / 2}\right)\left(s^{-r}\right)\right\} \\
& \alpha E^{(t / 2+r-1)} \text {. }
\end{aligned}
$$

Now, for the vibrational partition function for $v$ anharmonic vibrators

$$
\theta_{v}(E)=\underline{L}^{-1}\left\{\frac{1}{(s-m)^{v}}:=E^{v-1} e^{m E} .\right.
$$

The convolution equation describing the total density of states is

$$
\begin{aligned}
& \rho_{T O t}(E)=\int_{O}^{E} p_{v}(E-x) \rho_{t, r}(x) d x \\
& \approx e^{m E} f_{0}^{E}(E-x)^{v-1} e^{-m x} x^{(t / 2+r-1)} d x \text {. }
\end{aligned}
$$

The final approximation is to expand $e^{-m x}$ in a power series, keeping up through the quadratic term:

$$
e^{-m x}=\left(1-m x+\frac{m^{2} x^{2}}{4}\right)-. .
$$

This introduces an error of about 1 s. From here to the solution involves doing many integrals and collecting powers of E. Skipping all of this and going directly to the result: 


$$
\rho_{\operatorname{TOt}}(E)=e^{m E_{E^{\alpha}}} \int_{i=0}^{2} \frac{(-m E)^{i}}{i !} v_{j=0}^{1}(-1)^{j} \frac{(v j)}{(t / 2+r+i+j)}
$$

where $\left(\begin{array}{c}v-1 \\ j\end{array}\right)$ represents binomial coefficients and $a=$ $t / 2+r+v-1$. Remember that $E$ is shorthand for the energy $E_{a}-$ $\mathbf{E}_{\mathbf{u}} \cdot$

C.Testing the model on $\mathrm{Fe}(\mathrm{CO})_{5}$ and $\mathrm{Ni}(\mathrm{CO})_{4}$.

Fe and $\mathrm{Ni}$ were good candidates for testing the model. These metals have many lines in their atomic spectra, which allowed for averaging to improve the data. Also, information on their energy levels and transitions are anong the most reliable of all the transition metals. Finally, there are also reliable values for the bond strengths of their metal-carbonyl bonds, giving one less variable to worry about in the rate equation.

The spectra obtained from the reaction of rare gases with iron and nickel carbonyl can be found in Hartman (1979). Using the refinement of $\mathrm{I} / \mathrm{g}$ for the proper intensity of a state, the data were reanalyzed. Several scans were redone as a check on the reproducibility of the data, and the results were virtually identical to before. The attempt was also made to identify as many high-lying states as possible. The resulting populatiol plots are shown in Figure 4-1 for $\mathrm{Fe}(\mathrm{CO})_{5}$ and Figure 4-2 for $\mathrm{Ni}(\mathrm{CO})_{4}$. The rate plots are shown in Figures $4-3$ and 4-4; for a discussion of the deviation from this curve for some of the lower states 
of iron, see the next chapter. The open circles in the plots indicate that the result is from an unweighted single data point. The circles with crosses indicate the point is the average of 2 or more values with the standard deviation falling inside the limits of the circle. Finally, for averages with standard deviations falling outside the circle limits, bars are drawn to show the magnitudes of the deviations. Straight lines were drawn on the population plots through the region where a fit to one straight line was reasonable. The "temperatures" corresponding to these distributions were writter beside the curves.

For the rate plots, all of the data which were judged to fall on the curve were least-squares fit in terms of two variables $R$ and $P: \log ($ rate $)=K+P \times \log \left(E_{a}-E_{u}\right)$. The variable $K$ encompasses all of the neglected multiplicative physical constants and detection efficiencies. of more fundamental interest, $P$ is the power of the energy dependence which expresses the number of degrees of freedom that are active in the dissociation, using the harmonic co model.

The data base for Fe energy levels and transitions came Erom various sources. The best energy levels came from Reader and sugar (1975), and some state lifetimes were available in Corliss and Tech (1976). Researchers at NBS sent us preliminary results for the oscillator strength results from several different researchers, and our compilation came from a hierarchical ranking of the various 
sources, with older and less reliable data being calibrated and used when newer data were not availablel. Since that time, a publication based largely on the same collection of papers has appeared (Fuhr et al., 1981); these values have largely supplanted our tabulation. spot checks revealed that our values closely match the newly-compiled set.

For nickel, the latest tabulation of energy levels came from Corliss and sugar (1901). Host oscillator strengths came from Fuhr et al. (1981). Any other lines not listed there were then obtained from Corliss (1965) but with the calibration: $\log (g f)=1.13 \times \log (g f)$ corliss -0.185 .

The total bond energy for iron carbonyl is known fairly precisely, with values derived from disparate experimental methods yielding similar results. From thermochemistry experiments, it was calculated to be $6.0 \mathrm{ev}$ (cotton et al., 1959). Also, from a photoionization study, the value was found to be $6.25 \mathrm{ev}$ (Distefano, 1970). From our own laboratory, a very sensitive method for total bond energy yielded a value of $6.1 \mathrm{eV}$ (Horak, 1982). The best value of the total bond energy of iron carbonyl was taken to be $6.1 \mathrm{ev}$.

There are not as much data available for the total bond energy of nickel carbonyl, but two aifferent methods do

1The data of Blackwell et al. (1976, 1979) were used first. Data of Bridges and Kornblith (1974) were used next with 0.03 subtracted from their oscillator strengths. The same 0.03 was subtracted from May et al. (1974). The data of Holnik et al. (1970) were used with no corlections. Finally, all other data came from the massive tabulation of Corliss and Tech $(1968)$. Their data were adjusted using the following calibration: $\log (\mathrm{gf})=\left[1.18\left(\log (\mathrm{gf}) \mathrm{CET}^{+0.798-}\right.\right.$ $\left.3.311 \times 10^{-5} E_{1}\right)+0.1081$. 
yield simflar results. Thermochemical results indicate a value of $6.1 \mathrm{eV}$ for the total bond energy of $\mathrm{Ni}(\mathrm{CO})_{4}$ (Fischer et al.. 1957). The same photoionization study as for $\mathrm{Fe}(\mathrm{CO})_{5}$ again gave the value of $6.1 \mathrm{eV}$ for the total bond energy.

The metastable energy of argon was taken to be 93354 $\mathrm{cm}^{-1}$ based on a weighted average of the two states measured from Chapter III. No detailed analysis of relative proportions of energy carriers was performed for neon and helium. Estimates of their energies were taken to be 134043 $\mathrm{cm}^{-1}$ for neon and $161000 \mathrm{~cm}^{-1}$ for helium. Thus, the available energy for both the irnn and nickel carbonyl reactions ranged from $\sim 44000 \mathrm{~cm}^{-1}$ for argon to $\sim 112000$ for helium.

Using this information, the data shown in Figures 4-3 and 4-4 were $f$ it to the rate equation above; only states over $25000 \mathrm{~cm}^{-1}$ were fit for iron since collisional effects jumbled the rate values for the lower states. The following power dependences resulted:

Fe

$\mathrm{Ni}$

\begin{tabular}{|c|c|c|}
\hline $\mathrm{Ar}$ & $4.81 \pm 0.32$ & $3.40 \pm 0.48$ \\
\hline Ne & $5.09 \pm 0.34$ & $5.09 \pm 0.31$ \\
\hline He & $6.38 \pm 0.69$ & $6.20 \pm 1.23$ \\
\hline
\end{tabular}

The larger uncertainty in the helium results could be due to several factors. First of all, the overall production of metal states was lower than for argon, causing the effects 
of normal fluctuations in the flame intensities to be more significant secondly, the possible presence of different energy carriers such as helium ions could complicate the energy dependence. Also, ionization of the metal could be occurring in some unknown way, although no fluorescence of any ions was observed for any case. Finally, since the rate of decrease of state production at higher available energies is much less than for cases with lower available enerzies as with neon or argon, the scatter in the data is more important, due to a smaller range in rates.

It remains to consider different possible combinations of active degrees of freedom to see which dissociation mechanism best describes the observed power dependence. For a general. carbonyl $M(\mathrm{CO})_{n}$, there are $3+6 n$ total degrees of freedom, corresponding in the separated metal atom and co molecules to $n$ Co vibrations, $2 n$ co rotations, and $3 n+3$ total atomic and molecular translations. The total number OE degrees of freedom is 33 for $\mathrm{Fe}(\mathrm{CO})_{5}$ and 27 for $\mathrm{Ni}(\mathrm{CO})_{4}$. There is no way though to rationalize a dissociation where all 33 of the $\mathrm{Fe}(\mathrm{CO})_{5}$ modes or all 27 of the $\mathrm{Ni}(\mathrm{CO})_{4}$ modes would be active. This would require total dissolution of the co's into component atoms, a process for which there is no evidence nor enough energy to accomplish. The bighest active number of degrees of freedom that is reasonable corresponds to the case of slow, sequential loss of co's from the central metal atom. This sequential loss would impart rotational motion to the co's plus translation in 3 
dimensions. In the center of mass frame, this could result in up to $3 n$ translations, $n$ vibpations, a. 2 n rotations, or $\mathrm{E}^{21.5}$ for $\mathrm{Ee}(\mathrm{CO})_{5}$ and $\mathrm{E}^{17}$ for $\mathrm{Ni}(\mathrm{CO})_{4}$. However, even for a fully sequential dispersal of energy, due to the constraints of the molecular geometry, probably not all rotational modes could be fully active; the first and the last co's could be shot off directly so they would not rotate. Then the dependence would be down to around $\mathrm{E}^{17.5}$ for $\mathrm{Fe}(\mathrm{CO})_{5}$ and $\mathrm{E}^{13}$ for $\mathrm{Ni}\left(\mathrm{CO}_{4}\right.$

In contrast to this fully sequential dissociation, occurring slowly enough to allow for molecular rearrangement, the concerted, or rapidly sequential model is much more restricted. Since all co's receive impulses at once, the only possible direction of departure is along the line of the M-CO bond, causing no rotational motion and translation in only on direction, the direction along the axis of the M-CO bond. In addition, since the co's leave as one, there is no net force on the metal atom and the metal atom remains at the certer of mass. This model suggests $n-1$ translations, $n$ ibrations, and no rotations active, resulting in a power dependence of $E^{6}$ for $\mathrm{Fe}(\mathrm{CO})_{5}$ and $E^{4.5}$ for $\mathrm{Ni}(\mathrm{CO})_{4} \cdot 1$

The $f i t$ of the data from $n i c k e l$ and iron carbonyl dissociations to a power dependence most closely matches the restrictive, concerted model. For iron and nickel carbonyl

I thank Henry Luftman for his useful thoughts on detcrmining active degrees of freedom for the different cases. 
both, the observed power dependence with argon is a factor $1.2 \pm 0.4$ powers more restrictive than the model suggests. This could be due to an incomplete transfer to the vibrational modes. The consistency between the two cases lends credence to the validity of applying this model to similar carbonyls in Chapter VI where their total bond energies are not well known.

The power dependence increases for neon and helium. For neon, the results $l i e 0.9 \pm 0.3$ and $1.2 \pm 0.3$ units below those predicted for iron and nickel carbonyl respectively. For helium, the results for iron carbonyl are $0.4 \pm$ 0.7 above the predicted and for nickel $1.7 \pm 1.2$ above. The general trend of increasing power dependence in the cases of larger available energies is consistent with the vibrational modes becoming more fully active.

The data for helium and nickel however could contain a systematic error due to the possibility of cascading. That is, with many states produced all the way up to near the dissociation limit for these two cases, many have rapid transitions down to lower states which can then fluoresce to even lower states. The increase in population for these intermediate states would give an erroneously high formation rate, and would cause the power dependence to appear too high. It would be very difficult to account for and subtract off all of the cascaded intensities although in principle it could be done. But since all the higher states were never completely assigned, it would be hard to get it 
al. Nevertheless, cascading still should be a minor effect, since the highest states are minor compared to the .. lower. One advantage for argon is that the available energy "is low enough that no cascading can occur to states that are fluorescing. 
Figure 4-1. Population of atomic iron states from the dissociation of $\mathrm{Fe}(\mathrm{CO})_{5}$ by the quenching of argon, neon, and helium metastable states. The temperature arises from the distribution of the states through which a line is drawn. open circles on the plot indicate the result of a single data point. circles with crosses indicate the average of two or more points where the standard deviation falls within the circle limits. Larger standard deviations are drawn on the graph. 

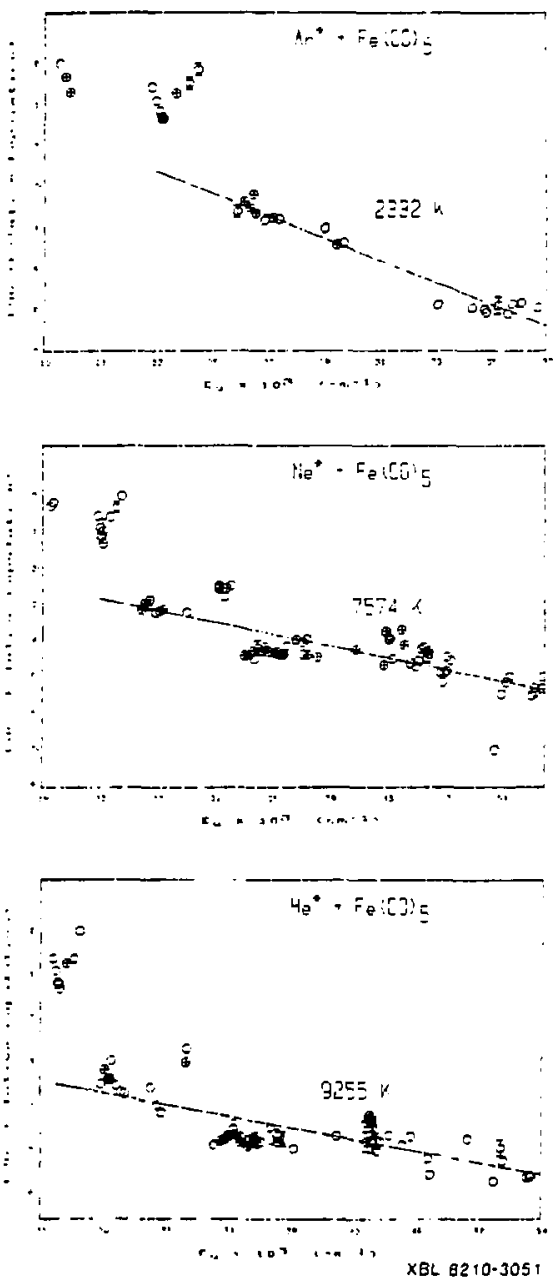

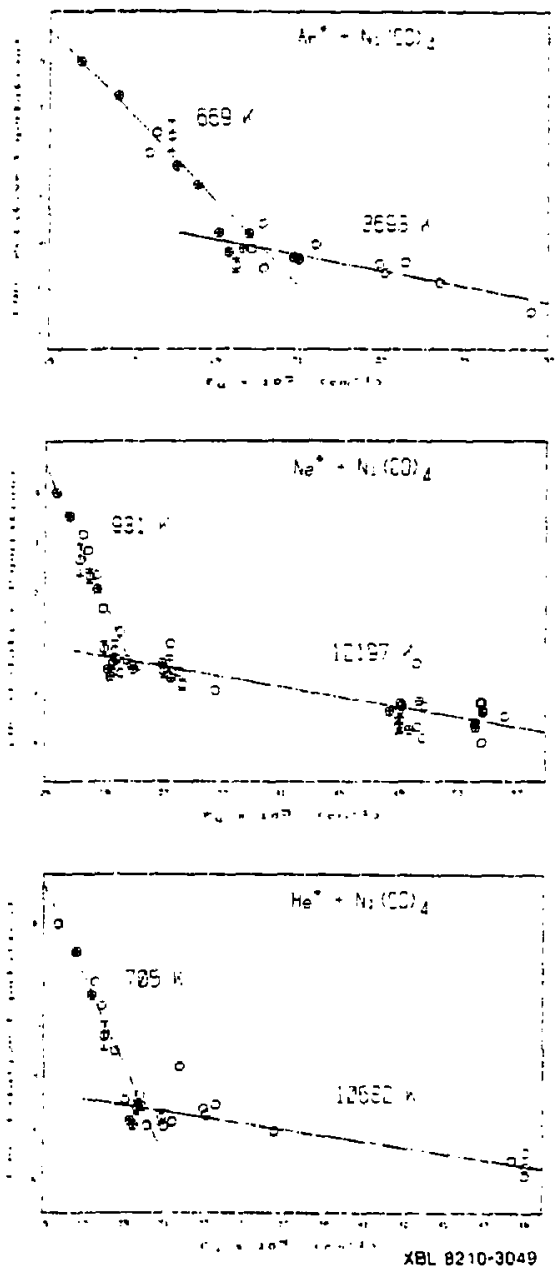

Figure 4-2. The population of nickel states resulting from the quenching of argon, neon, and helium by $\mathrm{Ni}_{(\mathrm{CO}}{ }_{4}$. symbols as in Fig. 4-1. 

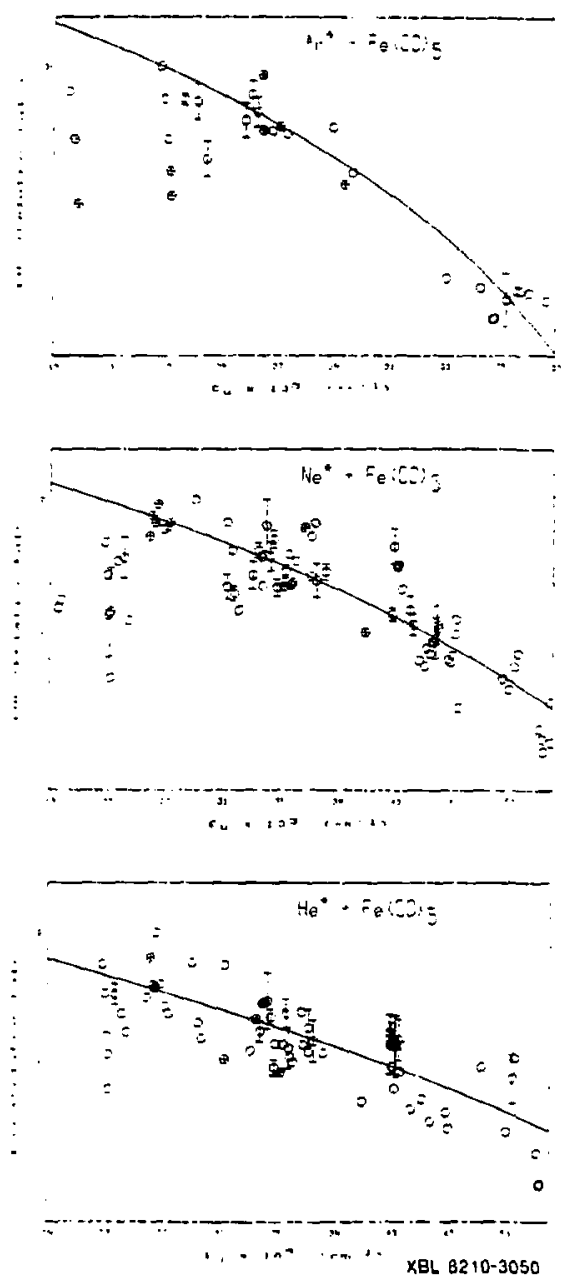

Figure 4-3. Rates of formation of lron states from using argon, neon and helium. The curves are fits to all but the long-lived, lower states. Symbols as in Fig. 4-1. 

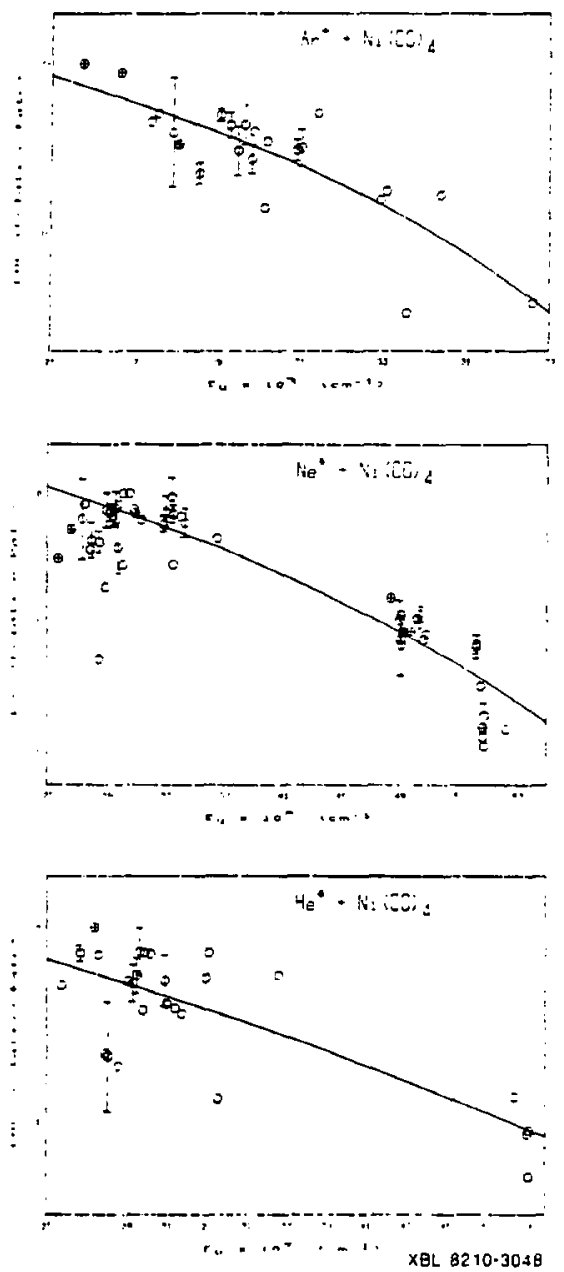

Figure 4-4. Rates of formation of nickel from argon, neon and helium. Symbols as it, Fig $4-1$. 


\section{Chapter V \\ Pressure Dependence of Long-lived Fe States}

Looking at the plots depicting the formation rates of iron or nickel (i.e. Figures $4-3$ and 4-4) shows that the only states, if any, which significantly deviate from the overall smooth downward curve are the lowest-lying states. This could be due to a real formation rate discontinuity, a process for which there is no ready explanation, or it could be due to the possibility that the intensities monitored in these cases do not in fact correspond to the true rate of formation for the states. Referring to eq. 4-2, the assumption is that the only channel for state depopulation is fluorescence. If there were any other non-fluorescent channels, they would be undetected and the result would be an erroneously small formation rate.

The common factor of all these deviating states is that they are all much longer-lived than the states lying higher in energy. The energies and lifetimes of these levels for iron and nickel are shown in Tables IV and V. This suggests then that collisional deactivation could be occurring for these states. That is, since they are much longer-lived, they suffer many more collisions, entering the realm where quenchers of reasonable efficiency can cause depopulation of the level.

The literature on intramultiplet relaxation is not large, and the theory for predicting the relative magnitudes for the quenching by different gases is not well-developed 
(e.g. Callear, 1975, 1978; King and Setser, 1976; and Yardley, 1980). Generally though, the less energy to ba carried away, the more favored is the process; the larger in size the quenching molecule, the more effective it is at quenching. This is due to the increased density of states of larger molecules which allows for more easily resonant energy transfer.

One important study was the flash photolysis of the intramultiplet relaxation of the $a^{5} \mathrm{D}$ ground state multiplet of Fe (Callear and Oldman, 1966, 1967) where the crosssection for the conversion of ${ }^{5} \mathrm{D}_{3} \cdot{ }^{5} \mathrm{D}_{4}$ was studied. This process is exothermic by $416 \mathrm{~cm}^{-1}$. For argon as the quencher, the cross-section w.s only $4.1 \times 10^{-4} \mathrm{~A}^{2}$ which alone is not sufficiently large to account for the observations of the current experiment. However, our average energy spacings are generally less, and as little as 95 $\mathrm{cm}^{-1}$.

An experiment to determine if indeed the long-lived states were being collisionally quenched involved measuring the intensities of these long-lived states as a function of pressure, with the limit of zero pressure yielding the unquenched, true intensity and hence the true rate of formation. Measuring the change in intensity of the one long-lived state alone was not sufficient due to the change in many other variables with the change in pressure. That is, metastable production and metal atom formation are also pressure dependent, so the intensity is a eunction of many 
variables and change is not due simply to the quenching of a state. In addition, as the pressure changes, so does the flame shape, and the intensity could be dependent upon that as well. Therefore, the intensity of the long-lived state had to be calibrated aguinst all of these other effects. The easiest way to do this was to monitor in addition the intensity of a short-lived state which should express all the same experimental variables except the quenching since the state radiates fast enough so that the quenching is negligible.

The experiment was difficult for other reasons as we11. One was that the zero pressure limit was not attainable since the discharge could not be sustained below about 0.2 torr. In addition, the metastable production was already very low by 0.35 torr. In this lower-pressure range, the metastable production curve dropped so fast that any fluctuation in the argon pressure could have caused a large error. In essence, the data had to be extrapolated downward from the region where values could be obtained. Also, as discussed previously, it was hard to monitor carbonyl pressures precisely. Finally, the data was errorprone since the desired trend was derived from dividing two very large values to obtain a curve expressing a smaller effect.

Without lengthy and difficult kinetic development needing state quenching constants which weren't even available, it was not obvious what the pressure-dependence 
of the intermediate states of a multiplet should even be. However, the highest energy level of a multiplet could only lose population to the lower states and its pressure dependence should be straightforward. But, because the highest states could only lose population from the effects of collisional quenching, they were also the lines with the smallest intensities. In addition, since these states also had low-J values, the intensities were even lower due to the smaller degeneracy of states compared to the lower-lying, higher-J states. Because of these two effects, you were Eated to spend your time dealing with a very weak IIne.

originally, it was hoped that accurate enough data could be obtained so that precise quenching rate constants couid be obtained. In theory it was a straightforward process; in practice, due to the inherent problems outlined above, the experimental data were inescapably vague. After what in hindsight now appears to be an excessive amount of time spent trying to coerce the experiment to yield the elusively consistent data by taking more and more data points with longer and longer time-averaging, the original expectations had to be sualed back to just demonstrating in a reproducible fashion that the calibrated intensities for the long-lived states did in fact climb with diminishing pressures.

The understanding of the guenching process improved considerably during the course of the time spent studying the pressure-dependent intensities. Since argon was higher 
in pressure by approximately 1000-fold over iron carbonyl, it was first assumed that the argon could be the only significant quenching channel. The early experiments were conducted by optimizing the flame intensity through adjusting the carbonyl pressure and monitoring the different pressures of argon. For the higher pressures of argon, the carbonyl pressure needed to maximize the intensity was largely constant, so that the overall upward trend in the intensity of the long-lived state due to the diminishing of the argon quenching channel was observed as the argon pressure dropped. However, at lower argon pressures, the variation in $\mathrm{Fe}(\mathrm{CO})_{5}$ pressure to maximize the flame intensity was much greater. As a result, there was a very large scatter in intensities for a small range of argon pressures. Originally, this was thought to be due to the low signal at these pressures. Finally though, the logical jump was made to realize that the $\mathrm{Fe}(\mathrm{CO})_{5}$ was also quenching the long-lived metal states, and, in fact, much more efficiently than the argon was. A new, and much more tedious experiment was conducted where, in addition to the argon pressure, the iron carbonyl pressure was monitored to $y$ ield an intensity-contour curve in three dimensions.

The Jarrell-Ash/DARSs system was, in theory, ideally suited for this experiment since, with its range of $600 \mathrm{~A}$ of simultaneous monitoring, all the lines of interest could be observed under the same conditions. The region chosen for study was in the range of 3800 to 4500 A which included a 
series of 1 ines emanating from the ${ }^{7}{ }^{\circ}$ as well as several lines from short-lived states which could be used as standards. The contour curves obtained did indeed show a steady upward increase in the intensities as both of the pressures were lowered, but the data were not extremely accurate. The big problem with this system, as mentioned in Chapter II, was the lack of sensitivity of the DARSS. Even with long averaging times as compensation, the accuracy was never very good.

The photomultiplier tube proved much more sensitive. Instead of simultaneous monitoring, one had to average one line at a given pressure and then go quickly to the other line for averaging. Then, as a check, the first line was reaveraged to ascertain that the conditions had not changed in the interim. Using the 3886.3 A line from the ${ }^{5} D_{3}^{0}$ state (1ifetime $=8.7 \times 10^{-8} \mathrm{sec}$ ) as the standard line and 4489.7 A from the ${ }^{7} F_{1}^{\circ}$ state as the long-lived state, Figure 5-1 demonstrates the upward trend in the intensities.

Although no rate constants resulted, the data showed that both argon and iron carbonyl provide significant quenching channels for the intramultiplet relaxation of some of the long-lived states of iron. Also, the quenching constant for tron carbonyl is at least 100 times higher than that for argon since similar results were obtained for argon in the torr range as for iron carbonyl in the micron range. This agrees with Callear's results (Callear and oldman, 1967) for the ground state relaxation. He lists an 
anomalously high cross-section of $23 \mathrm{~A}^{2}$ for the quenching of the $a^{5} D_{3}$ state by $F e$ itself. What he was probably observing instead of Fe was the efficient quenching by $\mathrm{Fe}(\mathrm{CO})_{5}$ that remained in small amounts after the flash photolysis.

There is also other support from more recent studies for the assumption that the long-lived states are being collisionally-quenched. The support comes from several molecular beam experiments where, due to the singlecollision conditions, no collisional quenching could be occurring. In a crossed-beam of $\mathrm{Ar}$ and $\mathrm{Fe}(\mathrm{CO})_{5}$ (Snyder et al., 1980), lines arising from the highez-lying ${ }^{7} \mathrm{~F}_{3}^{\circ}$ and ${ }^{7} \mathrm{~F}_{2}^{\mathrm{O}}$ states were strong lines for them whereas for us they were very weak. In another beam experiment (Kobovitch and Krenos, 1981), all the levels of the ${ }^{7} F^{\circ}$ multiplet observed vere produced at approximately the same rate, as our statistical model would predict. These results, along with our own study conclusively demonstrate that the deviation from the rate plot is caused by collisional quenching.

Callear saw for the ground state term of $\mathrm{Fe}$ a rapid Boltzmann population averaging for states which lay closer than the energy available at 298K, namely $206 \mathrm{~cm}^{-1}$. This averaging occurred at a much faster rate than the relatively slow relaxation of $D_{3}+D_{4}$, separated by $416 \mathrm{~cm}^{-1}$. The same situation appears to be true for our studies of the ${ }^{7}{ }^{\circ}$ term. Here, all of the levels are separated by less than $206 \mathrm{~cm}^{-1}$, and Boltzmann averaging occurs for all of the levels. Since $\left[I_{i u} \cdot A_{u}\left[M^{\star}\right]\right.$, the equation to calculate the 
ratios of the steady-state intensities expected for a Boltzmann distribution is

$$
\frac{\Sigma I_{i u, J}}{\Sigma I_{i u, J-1}}=\frac{A_{u, J}}{A_{u, J-1}} e^{-(\Delta E / k T)}
$$

where $\Delta E$ is the spacing in $\mathrm{cm}^{-1}$ between the adjacent $J$ states. The calculated values for the levels of iron and nickel are shown in the third column of Tables III and IV. Listed in the fourth column are our observed rate ratios, averaged where possible for the results from argon, neon, and helium. The agreement between the observed ratios to the ratios expected for levels that are Boltzmann-averaged for the ${ }^{7} \mathrm{~F}^{\circ}$ term of $\mathrm{Fe}$ is quite remarkable. 1

The results indicate that for all the long-lived states where spacing is on the order of $k T$ or less, the states Eeach a relative Boltzmann average amongst themselves. As is expected, for the cases with states with larger energy spacings, the intramultiplet relaxation does not reach a Boltamann distribution on the time scale of the state lifetimes. The wide standard deviation that exists for the cases of the different rare gases quenching the more widelyspaced ${ }^{7}{ }^{\circ}{ }^{\circ}$ multiplet of iron and all of the long-lived levels of nickel Indicates that these three gases quench with alfferent efficiencles, and the true ratios of state intensities as a result of the quenching by these three

lThanks to Kobovitch and Krenos (1981) for pointing this out. 
gases should not be identical.

Although the data weren't precise enough to yield rate constants for the rare gas and carbonyl quenching charinels, an estimate was made for the composite constant for the combined effects of quenching by all quenchers at the pressure at which the experiment was run. From equation 4-3, the expression for the rate of formation of a metal state with no quenching present, and hence the rate that is plotted is

$$
\mathrm{K}_{\mathrm{f}}^{\mathrm{plot}}=\mathrm{A}_{\mathrm{U}}\left[\mathrm{M}^{\star}\right]
$$

But the real formation rate must take into account the quenching, which is occurring at a total rate of $\mathrm{K}_{\mathrm{Q}}$

$$
\mathrm{R}_{f}^{\text {real }} \times\left[M^{*}\right]\left(A_{u}+K_{Q}\right)
$$

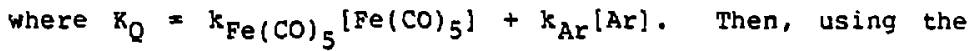
best least squares fit from the rate model of the last chapter to get an estimate for the true formation rate for a state with the same energy, the value of $k_{Q}$ can be found Erom

$$
K_{Q}=A_{u}\left(\frac{R_{E}^{\text {real }}}{K_{f}^{p l o t}}-1\right)
$$

For the iron states produced from collisions with argon, the average $K_{Q}$ for the two Boltzmann-averaged multiplets are $(3.9 \pm 0.5) \times 10^{6}$ for ${ }^{7} D^{\circ}$ and $(1.0 \pm 0.2) \times 10^{7}$ for $7_{F^{\circ}}$. For the ${ }^{7}{ }^{\circ}$ multiplet which has not averaged out, the 
values range from $6 \times 10^{5}$ to $7.2 \times 10^{6}$. Making the assumption that $\mathrm{k}_{\mathrm{Fe}(\mathrm{CO})_{5}} \sim 1^{100 \mathrm{k}_{\mathrm{Ar}}}$ and with typical conditions of 1 torr of argon and $10 \mathrm{~m}$ rons of $\mathrm{Fe}(\mathrm{CO})_{5}$ gives an estimate of $k_{\mathrm{Fe}}(\mathrm{CO})_{5}$ for intr= siiflet guenching to be on the order of 200 to $3000 \AA^{2}$ ine hard sphere collision cross-section for the interaction of $\mathrm{Fe}$ and $\mathrm{Fe}(\mathrm{CO})_{5}$ is approximately $300 \mathrm{~A}^{2}$. The even larger cross-sections than hard sphere for this Fe$\mathrm{Fe}(\mathrm{CO})_{5}$ interaction could be explained by both the larger effective diameters that the excited Fe states have compared to the ground state thus making the true hard sphere crosssection larger than $300 \mathrm{~A}^{2}$, and the presence of longerrange, attractive interactions that could be taxing place between the iron atom and the carbonyl, including possibly dipole or quadrupole interactions. Nevertheless, these estimates show that iron carbonyl quenches iron states with a very high efficiency. 


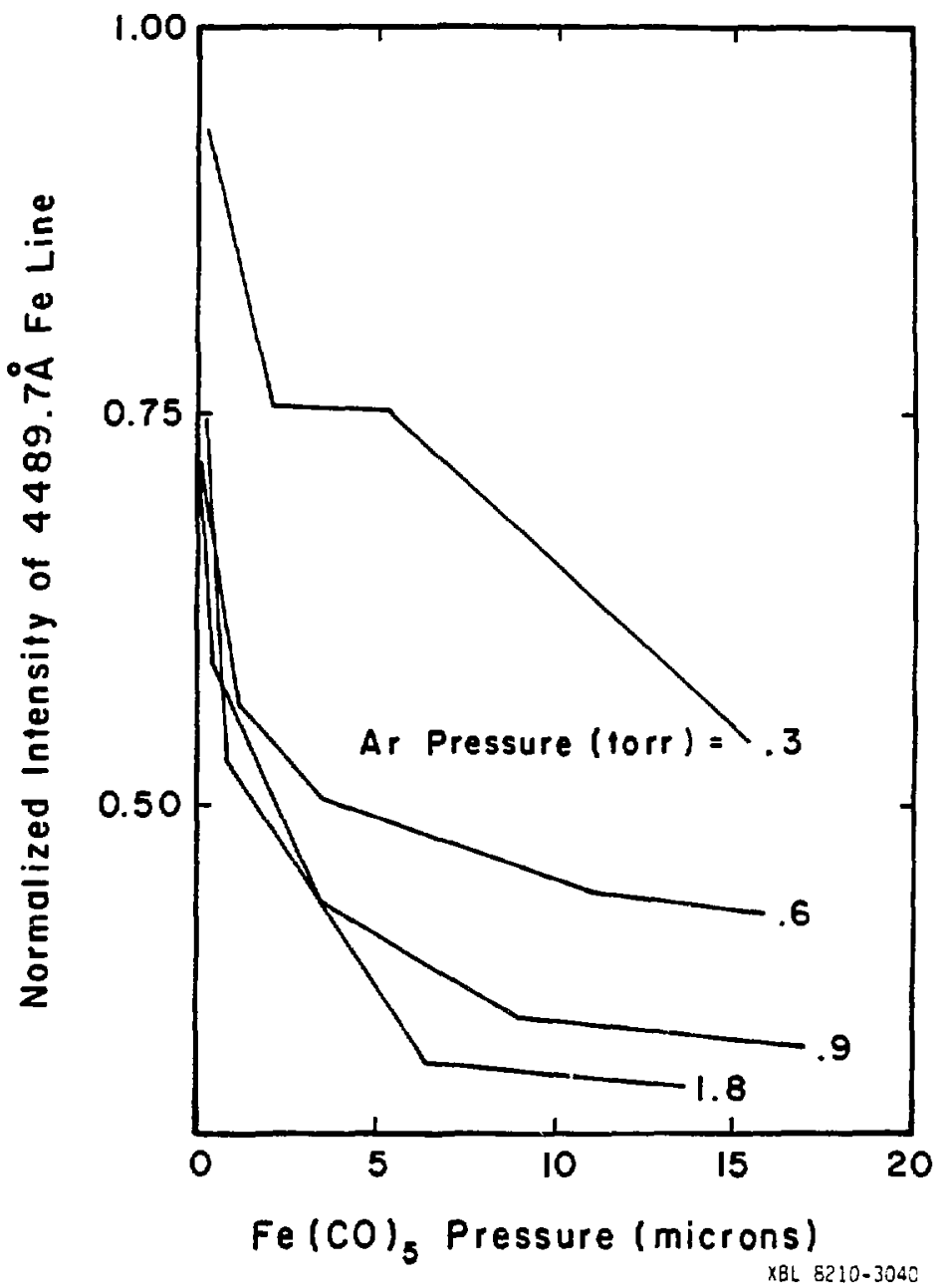

Figure 5-1. Intensity ratios at different argon and $\mathrm{Fe}(\mathrm{CO})_{5}$ pressures of the 4489.7 Fe line of a long-lived state divioed by the $3886.3 \mathrm{Fe}$ line from a short-lived state. 
Chapter VI

Results with Other Carbonyls

The mechanism of metal atom production from the collisional quenching of metastable rare gases by iron and nicke: carbonyl was found to be general for other carbonyls and some associated compounds as well. For the solids, once the problem of low volatility was surmounted by more effective heating and pumping of the sample, metal spectra were obtained, though sometimes with much weaker intensities than for iron and nickel carbonyl.

Unfortunately, the lifetime and $A$ coefficient information is not as precise or as extensive for most transition metals as it is for nickel and iron. If no new tabulations were available, the tabulation by Corliss and Bozman (1962) was used for information on transitions and Moore (1971) was used for energy levels. Newer energy levels for cobalt were found in sugar and Corliss (1981), for chromium in sugar and Corliss (1981), and for manganese in corliss and sugar (1977). No newer energy levels were available for molybdenum. As for line transition data, newer data for chronium and manganese were in a tabulation by younger et al. (1978) and Fuhr et al. (1981) for cobalt. There were no newer values for molybdenum.

The best and latest values for bond strengths for many carbonyls and similar inorganic compounds are in a paper by Connor (1977), resulting rom his own as well as earlier thermochemical studies. The bond strengths came from calcu- 
lations based on the observed heats of formation for these compounds. Carbonyls and metal compounds are noted for forming a range of decomposition products. Accuracy suffers if inexact analysis of decay products in the calorimeter occurs. The final value for the total bond energy comes from calculating the enthalpy change for the process

$$
M(C O)_{n}[g, 298 K] \rightarrow M[g, 298 K]+n C O[g, 298 K]
$$

Additional uncertainty comes in from the value for the heat of formation of the gaseous metal.

Since reliable values for the total bond energy for these carbonyls are not as well known as for iron and nickel carbonyl, the rate model was used to give an estimate for the available energy and hence the bond energy from a fit in terms of $K$ and $E_{a}$ to $\log ($ rate $)=K+P \times \log \left(E_{a}-E_{u}\right)$, where the value of $P$ is determined by making analogies to results for iron and nickel. To fit the data in terms of $K$ and $E_{a}$ required using a non-linear least squares procedure. The method chosen was an iterative method whereby guesses for these two constants were input into a matrix equation based on an expansion in terms of the first derivatives of the Eunction:

$$
\Delta \underline{x}=\left(\underline{D}^{T} \underline{D}\right)^{-1} \underline{D}(\underline{f} \text { obs }-\underline{f} c a 1 c)
$$

where (Eobs-Ecalc) is a $1 \times n$ matrix of differences between the observed rate and the rate calculated from the current values of $K$ and $E_{a}$ for $n$ data points, $D$ is a $2 \times n$ matrix of 
the derivative of the function with respect to the two variables evaluated at their current values for each data point, $\left(\underline{D}^{T_{D}}\right)^{-1}$ is a $2 \times 2$ matrix which is the inverse of the product of the derivative matrix and its transpose, and $\Delta x$, a $1 \times 2$ matrix containing the corrections for $K$ and $E_{a}$ to be added for the next iteration. Also calculated were the correlation and standard deviation based on a linear approximation.

Except for the earlier study of $\mathrm{He}{ }^{*}+\mathrm{Mn}_{2}(\mathrm{CO})_{10}$, all of these experiments were done very recently. When they were done, the current cylinders of neon and helium contained high amounts of impurities, so much so that adequate concentrations of the metastable gases were not obtained. At this point, there was not sufficient time or finances to replace them with better supplies. So, only argon metastable state reactions were used for these newer studies. It would be useful to return to some of these compounds in the future to study their interactions with the higher-energy metastable rare gas states.

A. Argon + Chromium hexacarbonyl.

The spectrum of $\mathrm{Ar}+\mathrm{Cr}^{*}(\mathrm{CO})_{6}$ yielded atoraic lines in the range of 3600 to 4500 A as seen in Figure 6-1; there were also several other lines in the range of 5200 to 5400 A. The strongest 1 ine was about 2500 counts per second, located at $4254.4 \mathrm{~A}$ and coming from the $23499 \mathrm{~cm}^{-1} \mathrm{z}^{7} \mathrm{P}_{4}^{\circ}$ state. The population plot is shown in Figure 6-2, and the 
rate plot in Figure $6-3$.

The highest energy level confirmed for chromium was the $31280 \mathrm{~cm}^{-1} z^{5} \mathrm{~F}_{5}$ state which places an upper limit on the bond energy of $61600 \mathrm{~cm}^{-1}$ which compares to Connors" value of $54000 \mathrm{~cm}^{-1}$. The rate data were fit to an estimated power dependence of $\mathrm{E}^{6.3}$, which is 1.2 units less than the restricted model prediction for $M\left(\mathrm{CO}_{6}\right.$ of $\mathrm{E}^{7.5}$. This gives a value for the available energy of $44859 \pm 262 \mathrm{~cm}^{-1}$ which yields a total bond energy of $48285 \pm 262 \mathrm{~cm}^{-1}$ which falls a little less than Connor's value, placing it in the same range of bond energy as for nickel and iron carbonyl.

B. Argon and Molybdenum hexacarbonyl.

The spectrum of $\left.\mathrm{Ar}^{\star}+\mathrm{Mo}^{\mathrm{CO}}\right)_{6}$ was a relatively weak series of lines from 3750 to 5000 A and is shown in Figure 6-4; there were several additional lines from 5500 to 5600 A. The strongest line was at 5506.5 A arising from the $28924 \mathrm{~cm}^{-1} \mathrm{z}^{5} \mathrm{P}_{3}^{\circ}$ state. The population and rate plots are shown in Figures $5-5$ and $6-6$.

The population distribution was rather surprising in that there was a very clear temperature inversion for the levels lying 25000 to $33000 \mathrm{~cm}^{-1}$. Instead of indicating a different formation mechanism as was first thought, it just reflected the fact that the very lowest states are shorterlived than those lying above, and the population inversion merely reflected the longer lifetimes of the higher states. By $33000 \mathrm{~cm}^{-1}$ the states have once more become 
shorter-lived and the population begins to decrease.

The highest iine seen for Mo was the $35719 \mathrm{~cm}^{-1} \mathrm{z}^{5} \mathrm{~F}_{5}^{\circ}$ state, which means a total bond energy of less than 53425 $\mathrm{cm}^{-1}$, or $7.1 \mathrm{eV}$, which is already greatly less than the thermochemical value of $75815 \mathrm{~cm}^{-1}$. Applying the rate model using $E^{6.3}$ as for chromium and excluding the lower-lying long-lived states, the best fit gives a bond energy of 14929 $=11864 \mathrm{~cm}^{-1}$, an unacceptably low value. Between the weak signal, the few points included in the $f$ it, and the outdated line transition data used in the calculations, it's not very surprising that the resulting $f i t$ is a poor one. Fitting the data using the thermochemical value of 75815 gives a very low power dependence of $\mathrm{E}^{2.17 \pm .88}$, which would imply a very restrictive dissociation, if true.

The results for Mo( $\mathrm{CO})_{6}$ would seem inconclusive. For a bimolecular process, the upper limit of $7.1 \mathrm{eV}$ for the bond strength would be firm, as no other significant energy sources are avallable in such a gas phase study. If perhaps the process is not bimolecular, then the bond energy value would be wrong. A pressure dependent study of line intensities would settle the argument, as was done originally to establish the bimolecular nature for the dissociation of iron carbonyl.

c. Helium and Dimanganese decacarbonyl.

In this older study, a very weak spectrum of manganese lines was observed in the range of 2800 to 4800 A for He* + 
$\mathrm{Mn}_{2}(\mathrm{CO})_{10^{\circ}}$ No fluorescence was seen with argon or neon. There was no evidence of any metal molecular fluorescence, worth noting since the presence of a $\mathrm{Mn}_{2}^{*}$ fragment was thought to be a possibility. The strongest line was 350 counts from a set of lines at 4033 \& consisting of an unresolved set of three lines from the $z^{6} p^{0}$ multiplet. The feature that many of manganese's lower multiplets have similar energy spacings caused several sets of unresolvable lines. The population and rate plots for the four lines that could be assigned are shown in Eigures $6-7$ and 6-8. The highest observed state was the $44289 \mathrm{~cm}^{-1} \mathrm{z}^{4} \mathrm{~F}$. 3 level which gives ... upper limit to the bond energy of 116711 $\mathrm{cm}^{-1}$, a value which is consistent with the thermochemical value of 89527 .

Not knowing what the dissociation of $M_{2}(\mathrm{CO})_{10}$ is like and not having any other examples with which to compare it to meant that the rate model had to be applied with uncertainty. But since only four points were available the concern was merely academic since there was not much confidence in the results. Analogous to the restricted model previously developed, $\mathrm{Mn}_{2}(\mathrm{CO})_{10}$ would have an energy dependence of $-E^{9.5}$. Just for grins, the data was $f i t$ and the best fit gives a bond energy of $68861 \pm 12180$ which is at least ballpark to the thermochenical value. Using the thermochemical value gives an energy dependence of $5.2 \pm 1.2$ which is somewhat more restrictive than predicted. 
D. Argon and Cobalt tricarbonyl nitrosyl.

$\mathrm{Co}(\mathrm{CO})_{3} \mathrm{NO}$, a substituted carbonyl, is a liquid with a high vapor pressure. The spectrum with argon yielded lines in the range of 3000 to 4400 \& and is shown in Figure 6-9. There were several very strong 1 ines with the strongest of about 9000 counts coming from the $23856 \mathrm{~cm}^{-1} \mathrm{z}^{6} \mathrm{~F}_{4.5}^{\circ}$ state. The population and rate plots are given in Figures $6-10$ and 6-11.

The highest observed state was the $34352 \mathrm{~cm}^{-1}$ $z^{2} D_{1.5}^{\circ}$ state, so $E_{b}<58792 \mathrm{~cm}^{-1}$. No thermochemical data was available for this compound. Guessing at the rate dependence gives a value around $E^{4.5}$ since in the number of fragments it is most similar to $\mathrm{Ni}(\mathrm{CO})_{4}$. The rate dependence was fit using this power for the energy dependence and fitting only to the states above $28000 \mathrm{~cm}^{-1}$ since the lower states were long-lived and showed signg of collisional jumbling. The spacing for some of these levels is less than kT so Boltamann averaging could be occurring as it was for $\mathrm{Fe}(\mathrm{CO})_{5}$. A fit to $\mathrm{E}^{3.25}$ as for $\mathrm{Ni}(\mathrm{CO})_{4}$ gave $\mathrm{E}_{\mathrm{b}} \sim 50639$ $\mathrm{cm}^{-1}$, or $6.2 \mathrm{ev}$, consistent with the upper energy limit. This means that with an assumption of $\mathrm{N} i(\mathrm{CO})_{4}-1 \mathrm{ike}$ behavior. the bond energy is similar to $\mathrm{Ni}(\mathrm{CO})_{4}$.

E. Argon and Ferrocene.

Ferrocene, Fe( $\left(\mathrm{C}_{5} \mathrm{H}_{5}\right)_{2}$, is sandwich compound; that is, two rather large cyclopentadienyl rings are bonded sideways to the iron atom in the middle. It is a sublimable solid, 
giving slightly less vapor pressure though than the solid carbonyls tried.

The spectrum of $\mathrm{Ar}{ }^{\star}+\mathrm{Fe}\left(\mathrm{C}_{5} \mathrm{H}_{5}\right)_{2}$ gave very weak but recognizable iron lines as seen with the dissociation of iron carbonyl. The strongest line, as with iron carbonyl, was the 4375.9 A line from the $22846 \mathrm{~cm}^{-1} \mathrm{z}^{7} \mathrm{~F}_{5}^{\circ}$ state. The rate and population plots are shown in Figures 6-12 and $6-13$

The highest state observed was the $33695 \mathrm{~cm}^{-1} Y^{5} F_{5}^{C}$ state. The thermochemical value for the total bond energy is $49653 \mathrm{~cm}^{-1}$, or $6.2 \mathrm{eV}$, which is very close to the value for $\mathrm{Fe}(\mathrm{CO})_{5}$. Fitting to the rate model is difficult since there is no accounting for the possible excitation of the many different cyclopentadienyl vibrational modes. However, as opposed to the impulse that a co receives along its vibrational direction, the impulse that a $C p$ group would receive would be perpendicular to the major vibrations, coupling weakly perhaps to the out-of-plane bending modes. Excluding the long-lived states and using the thermochemizal value for the total bond energy, the power that fits the data best is $\mathrm{E}^{0.74 \pm .43}$ which is a dramatically lower energy dependence than seen previously. Excitation of only the two one-dimensional translational modes would yield a dependence of $E^{0.5}$ which lends support to a simultaneous bond breaking of the Fe-Cp bonds with 11ttle rotational or vibrational excitation. 
F. Other compounds tried.

Manganese cyclopentadienyl tricarbonyl, $\mathrm{MnC}_{5} \mathrm{H}_{5}(\mathrm{CO})_{3}$, is a liquid. As with the other liquids tried, a strong metal excitation was expected. However, in spite of being a liquid, the compound had no detectable vapor pressure even with heating, and there was no detectable fluorescence when aaded to a flow of metastable argon atoms.

Tungsten hexacarbonyl was also tried. Although a similar pressure was obtained as for the other solid carbonyls, no spectrum was seen for $\mathrm{Ar}^{*}+W(\mathrm{CO})_{6}$. The thermochemical bond energy is listed as $89527 \mathrm{~cm}^{-1}$ which, if true, would preclude the populating of any fluorescent states of tungsten. With the lowest possible level of tungsten that could fluoresce lying 19389 above the ground state, a lower limit on the bond strength would be set at 73800 or $9.1 \mathrm{eV}$. This would be an obvious candidate to try with neon or helium metastable states.

A comparison of bond energies estimates from the shermochemical experiments, rate calculations, and the highest states observed for the compounds mentioned in this chapter is shown in Table $v$. 


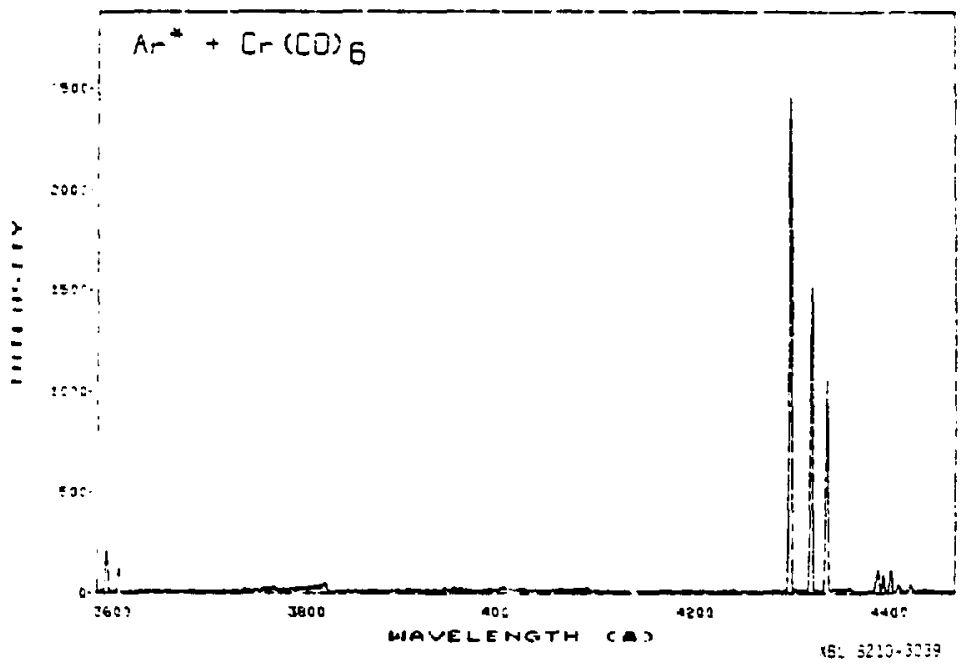

Figure 6-1. The spectrum of chromium lines from Ar + $\mathrm{Cr}\left(\mathrm{CO} 6_{6}{ }^{\circ}\right.$ The weak molecular emission at $3800 \mathrm{~A}$ is due to background $\mathrm{N}_{2}$. 


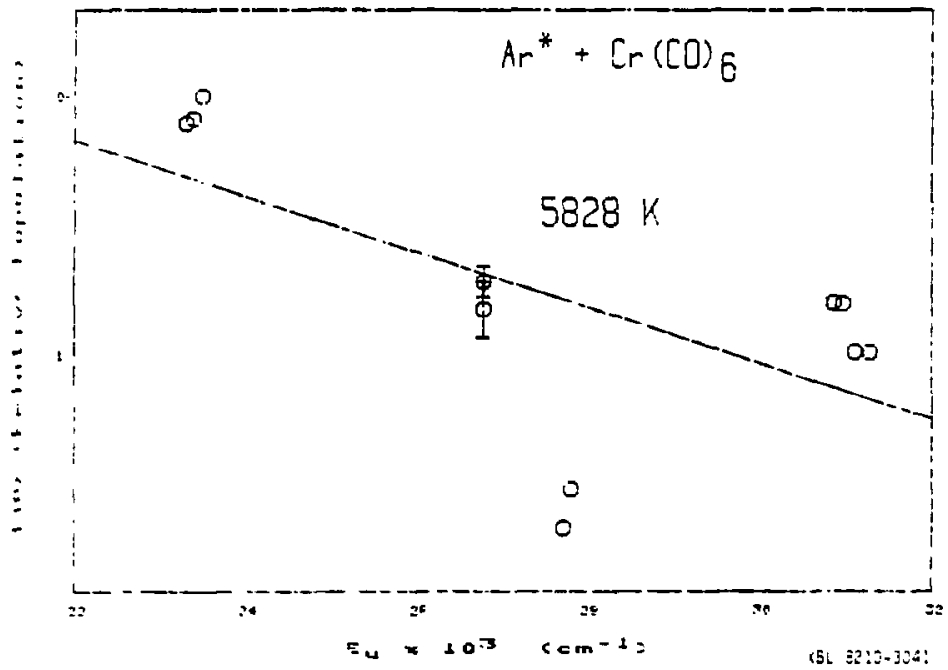

Figure 6-2. Populations of chromium states from Ar" + $\mathrm{Cr}(\mathrm{CO})_{6} \cdot$ Symbols as in Fig. 4-1. 


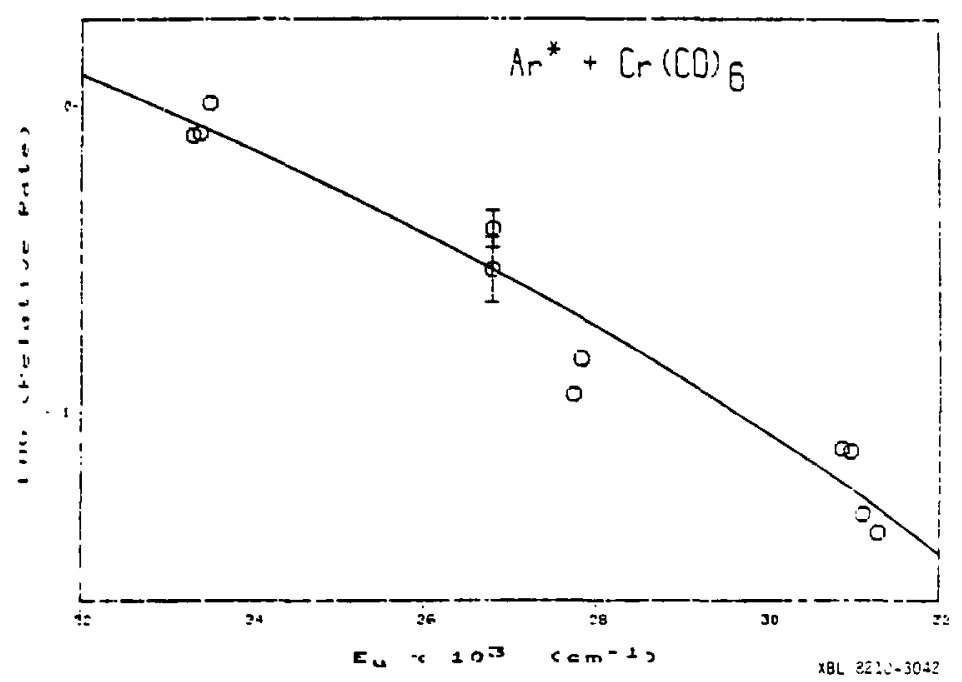

Figure 6-3. Rates of formation of chromium states from Ar* $+\operatorname{Cr}(\mathrm{CO})_{6} \cdot$ Symbols as in Fig. $4-1$. 


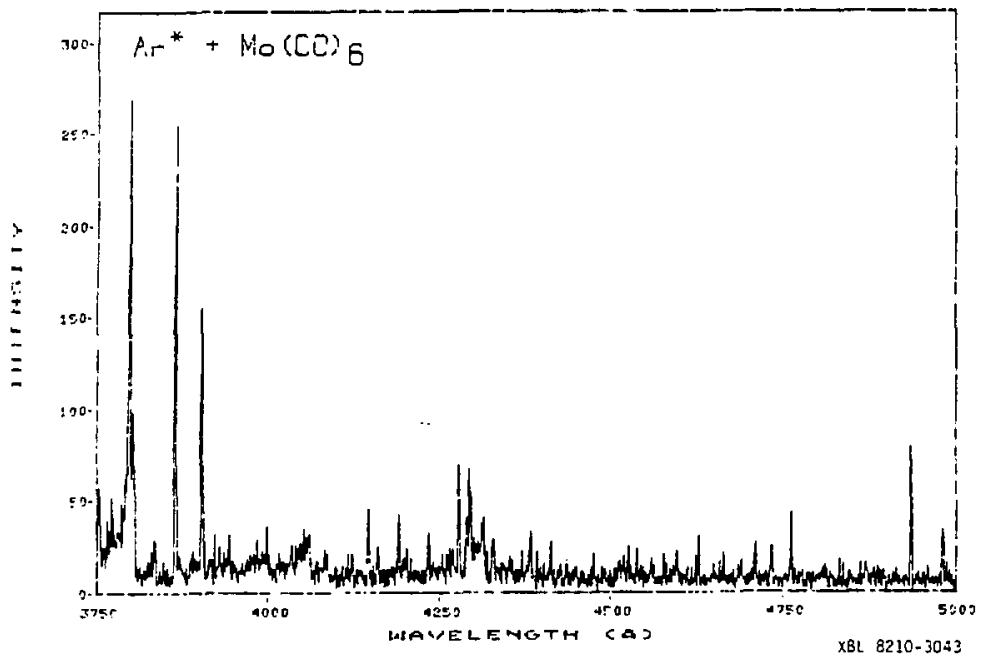

Figure 6-4. The spectrum of molybdenum lines from Ar ${ }^{*}$ MolCo $)_{0}$ The weak milecular structure is due to $\mathrm{N}_{2}$. 


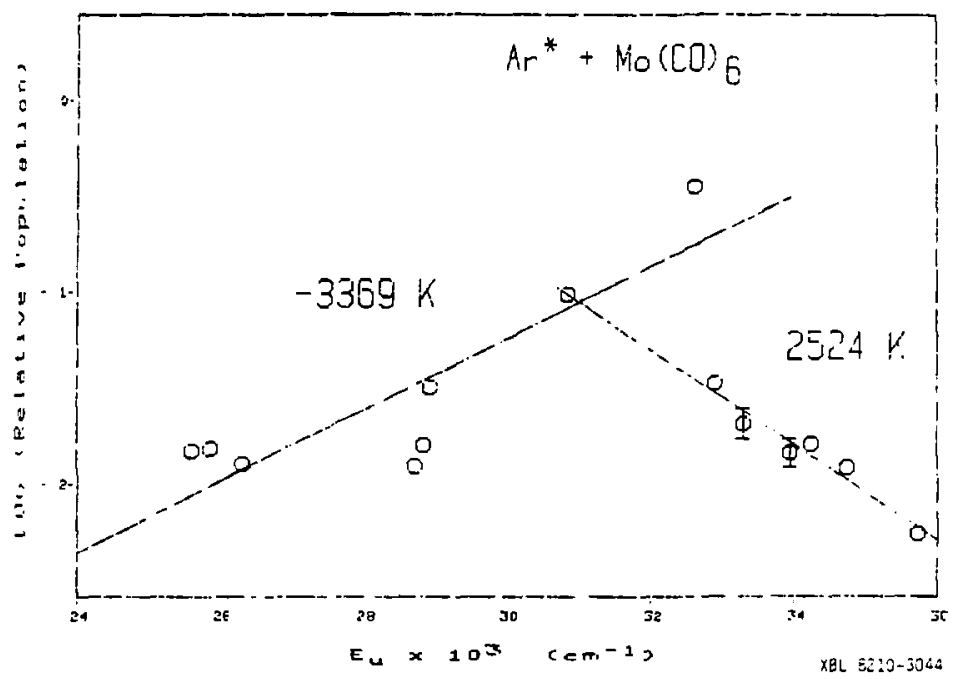

Figure 6-5. Populations of molybdenum states from $\mathrm{Ar}^{\text {* }}$ + Mo(CO) 6 . The negative temperature for the lower states indicates a population inversion for these states. Symbols as in Fig. $4-1$. 


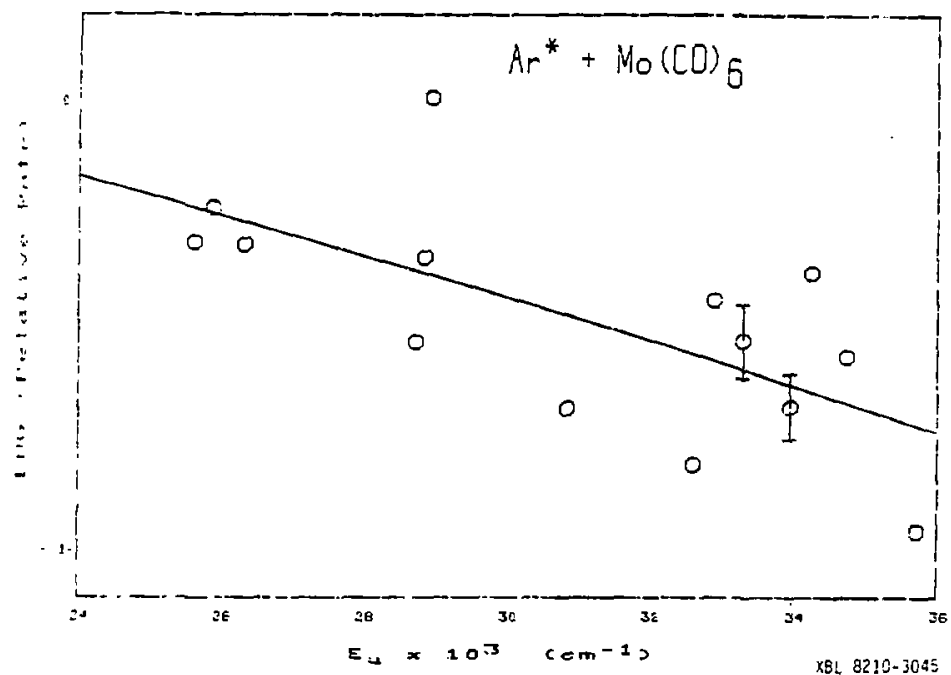

Figure 6-6. Rates of formation of molybdenum states Erom $\mathrm{Ar}+\mathrm{Mo}(\mathrm{CO})_{6} \cdot$ Symbols as in Fig. 4-1. 


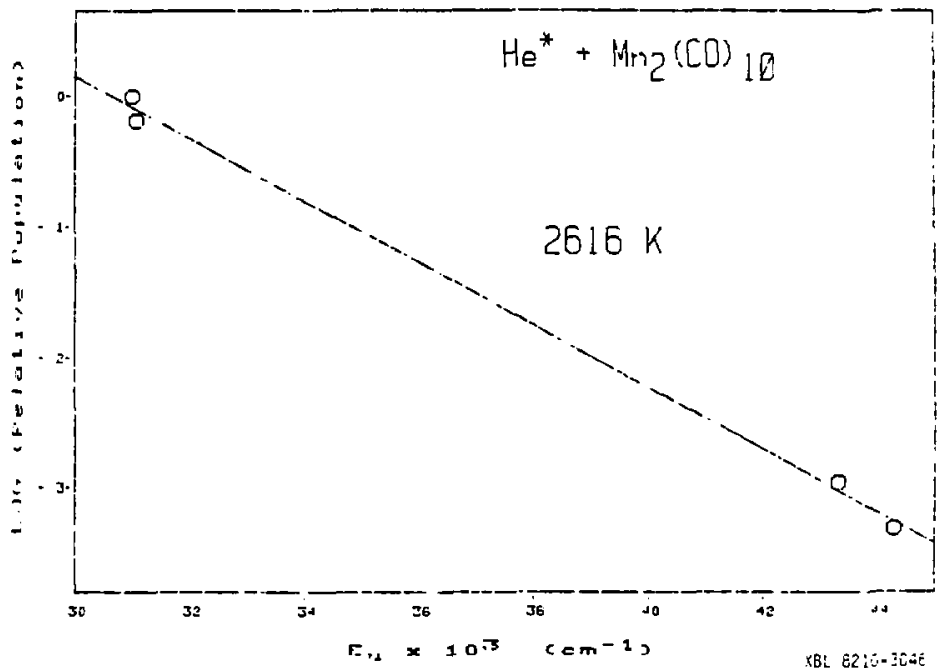

Figure 6-7. Populations of manganese states from $\mathrm{He}^{\star}+$ $\mathrm{Mn}_{2}(\mathrm{CO})_{10^{\circ}}$ Symbols as in Fig. 4-I. 


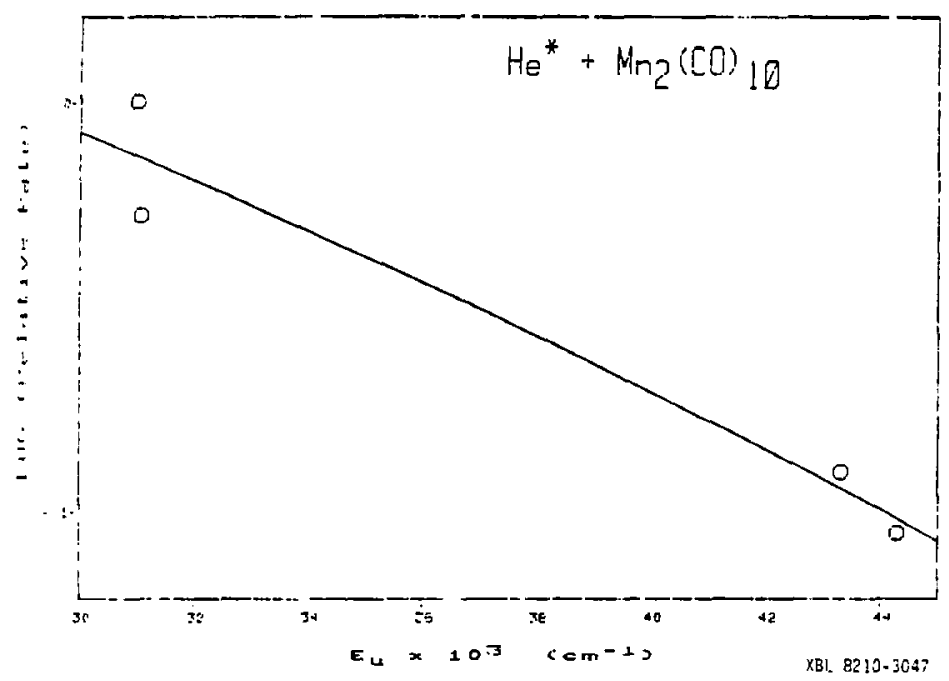

Figure 6-8. Rates of formation of manganese states from He* $+\mathrm{Mn}_{2}(\mathrm{CO}) 10^{\circ}$ Symbols as in Fig. 4-1. 


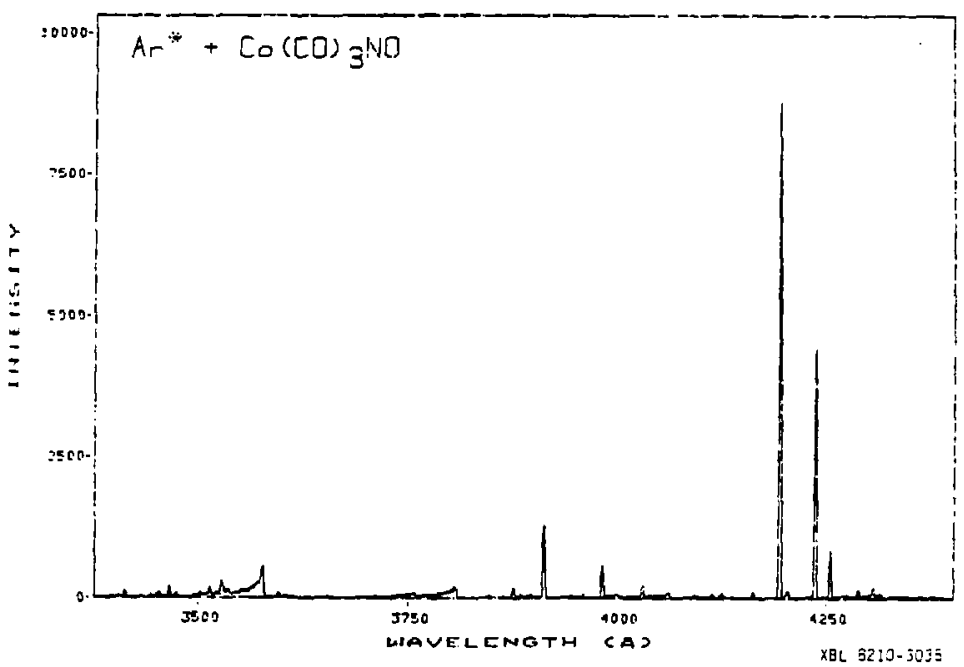

Figure 6-9. The spectrum of cobalt lines from $\mathrm{Ar}^{\star}$ + $\mathrm{Co}(\mathrm{CO})_{3} \mathrm{NO}$. The molecular structure is due to $\mathrm{N}_{2}$. 


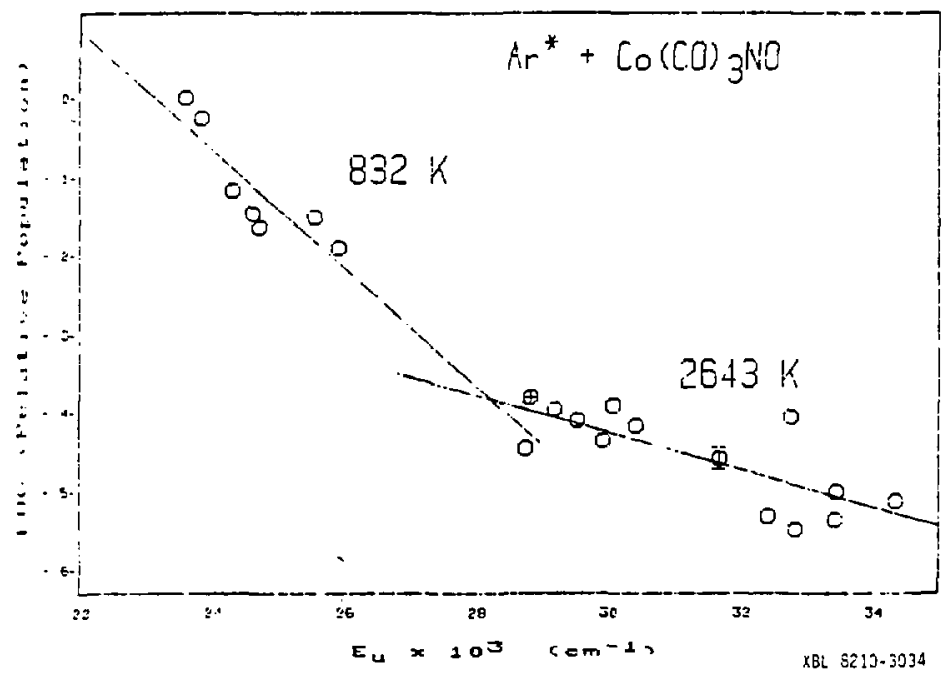

Figure 6-10. populations of cobalt states from $\mathrm{Ar}^{*}+$ $\mathrm{Co}(\mathrm{CO})_{3}$ NO. Symbols as in Fig. $4-1$. 


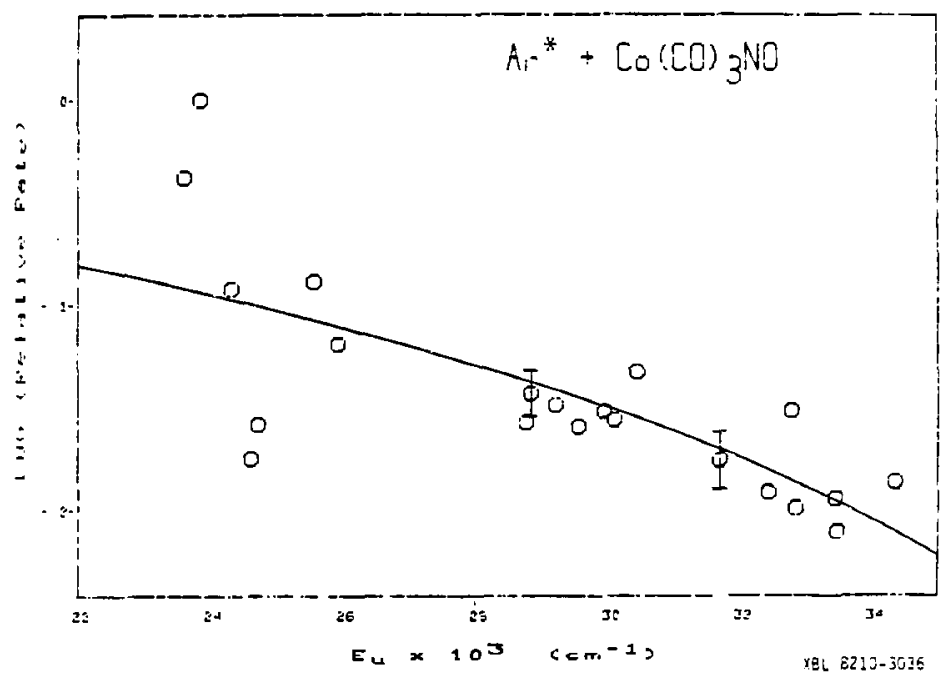

Figure 6-11. Rates of formation of cobalt states from $\mathrm{Ar}^{*}$ + Co(CO) ${ }_{3}$ NO. The deviating, long-lived states were not used in the curve-fit. Symbols as in Fig. 4-1. 


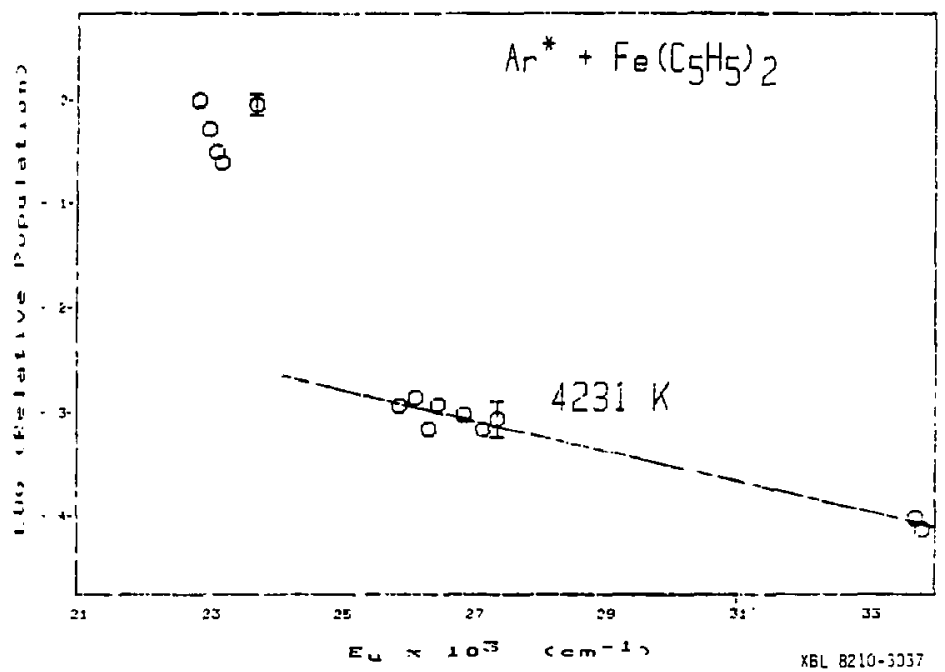

Figure 6-12. Populations of iron states from $A r^{*}+$ Fe $\left(\mathrm{C}_{5} \mathrm{H}_{5}\right)_{2}$. Symbols as in Fig. 4-1. 


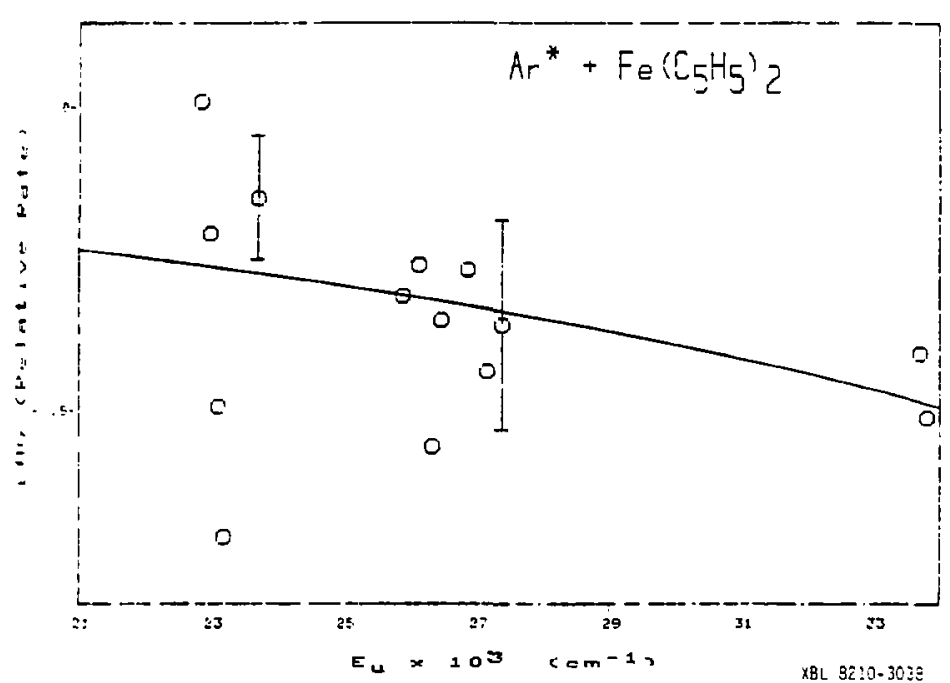

Figure 6-13. Rates of formation of iron states from Ar * $\mathrm{Fe}\left(\mathrm{C}_{5} \mathrm{H}_{5}\right)_{2}$. The lowest states are long-lived and are not included in the curve fit. Symbols as in Fig. 4-1. 
Chapter VII

Reaction Chemistry of Metal Aioms

having a concentration of metal atoms in the gas chase is a very appealing situation. With all of its orbitals in unbonded states, the metal atom is a very high energy species and thus is extremely reactive. There are many different reactions that could occur involving the atom, with many exothermic product channels existing in reactions with many reagents; this exothermicity comes about from the much lesser energy that the metal atom has as a result of bond formation. The metal atom has to be sufficiently high in energy to overcome the energy gain that results from the breaking of the bonds of the incoming reagent and replacing it usually with a higher energy species such as a radical. In addition to being exothermic, the exit channel has to have enough exira energy so that fluorescent products thit could be formed are in the range of energy that we can detect. The possibility of using metal atoms as a route to producing band structure for small inorganic molecules which may exist only in the gas phase was the motivation for this aspect of research.

Using $\mathrm{N}_{2} \mathrm{O}$ or $\mathrm{Cl}_{2}$ with the hopes of forming the oxide or chloride of iron or nickel is feasible since the enthalpy change for at least some of these processes is exothermic. The heat of formation data is not well established for gas Fisase species such as $\mathrm{FeO}$ or $\mathrm{NiCl}$, but at least for the provess forming Nio, 


$$
\mathrm{Ni}+\mathrm{N}_{2} \mathrm{O} \rightarrow \mathrm{NiO}+\mathrm{N}_{2}
$$

enough data exists to calculate the anthalpy change for the process; its value is $-63 \mathrm{kcal}$ mole $\mathrm{k}^{-1}$, or $3 . ; \mathrm{eV}$. Potentially, fluorescence from this system would be detectable.

Quite a lot of time was spent in trying different combinations of nickel and iron with reagents such as $\mathrm{Cl}_{2}$ and $\mathrm{N}_{2} \mathrm{O}$. The carbonyl was introduced either upstream for a flow in the interaction zone of primarily ground state metal atoms, or at the port directly orposite the monochromator to produce a mixture of ground and excited state metal atoms. The resulting plots were typically very complicated. The parent metal lines were usually still present to some extent. In adcition, some direct excitation of the reagent with the metastable rare gas state occurred, prociucing band structures of these reagents. In no caste was any metal molecular emission detected. With $\mathrm{Cl}_{2}$ as the reagent added to a flow of nickel, a very weak band structure occurred around 3900 to $4700 \mathrm{~A}$. This firje was thought to be $\mathrm{NiCl}$ fluorescence. However, the same structure was also later found in the spectra from $\mathrm{Ar}^{*}+\mathrm{CO}$, and with iron as well. Although neyer fully identified, this structure was most Iikely due to some very weak $\mathrm{N}_{2}(\mathrm{C}+\mathrm{B})$ bands from background nitrogen, and/or some high-vibrational states of the co Asundi band system $\left(a^{\prime} 3_{\Sigma^{+}} \rightarrow a^{3} \pi\right)$. The $\mathrm{Ni}(\mathrm{CO})_{4}$ was stored under co to inhibit dissociation so it was ine itable that some co would always be present. 
These operating conditions were fleeting in occurrence due to an unexpected result. Quickly upon inception of the flow of the carbonyl and the additional reagent, a vacuum deposition process began. Very fragile and detailed stalactite-like structures began forming from the walls and growing rapidly toward the middle. Although these conpounds were never analyzed tirey were attributed to metal oxides or chlorides resulting from the direct reaction of the carbonyl with the additional reagent. Although beautiful, all aesthetic senses aside, this made the whole experiment of incredibly short duration before the growth either obscured the monochromator's vision, or interfered enough with the flow patterns that intensities were diminished. The chamber had to be cleaned before any experiments could be resumed; the whole situation made this an impractical experiment.

This whole approach to reaction chemistry turned out to be too messy to work with. You were stuck with a background flow of carbonyl many times higher than the metal atoms produced. Whatever reaction might be occurring was occurring on a very small scale and was obscured by direct reactions which were occurring with the unreacted carbonyls or unquenched metastable rare gas atom concentration. 
Chapter VIII

Conclusions

The production of metal atoms from the energy transfer of the metastable energies of rare gases to metal carbonyls now appears to be a general process which will work for whatever carbonyis reach sufficient pressures in the gas phase. Results so far indicate that all metal states are produced up to the limit of energy remaining after all bonds have been broken, and produced at a rate determined by the energy of the upper state with no regard to spin multiplicities. The distribution of states, not being governed by selection rules as would be the case with excited state population by absorption from the grouns state, is a unique way to produce states which would be inaccessible from the ground state. In fact, this met.rod produces relatively high populations of these states for precisely the same reason, namely, that they are not quickly able to fluoresce to lower states, and the steady-state population builds. This high population of low, improbable states can exrose previously unknown behavior; for example, in the study of $\mathrm{Ar}^{*}$ + $\mathrm{Ni}(\mathrm{CO})_{4}$, several lines from two long-lived states of lickel were observed for the first time. The novel production of population inversions that sometimes results from the presence in some metals of long-lived states lying higher in energy than some shorter-lived states could perhaps be put to use in laser development, although the absolute soncentrations are only on the order of less than $10^{9}$ per $\mathrm{cm}^{3}$ 
since the primary state produced in all cases is the ground state itself.

It now appears that a wide range of inorganic compounds similar in bonding to the carbonyls will produce metal atoms. An advantage of the flowing afterglow technique is the very low amounts of carbonyl required to fully explore the dissociation of the carbonyl so that even rather rare and expensive compounds could be used. So far, it seems that metal stoms are produced by multiphoton methods whenever the afterglow pathway exists as well. Thus, compounds which have produced metal atoms from these multiphoton methods would be likely to produce metal atoms in the afterglow method as well. Results from these groups indicate that metal alkyls also dissociate in similar ways (Karny et al., 1978). From multiphoton work, metil atoms and ions have been seen from $\mathrm{Cr}(\mathrm{CO})_{6}$ and $\mathrm{W}(\mathrm{CO})_{6}$ (Gerrity et al., 1980), from ferrocene and nickellocene, $\mathrm{Ni}\left(\mathrm{C}_{5} \mathrm{H}_{5}\right)_{2}$, (Leutwyler et al., 1980); metal atoms and up to the trinuclear ions have been seen from $\mathrm{Mr}_{2}(\mathrm{CO})_{10}$ and $\mathrm{Fe}_{3}(\mathrm{CO})_{12}$ (Leutwyler and Even, 1981), and neutral $\mathrm{Co}_{2}$ from $\mathrm{Co}_{2}(\mathrm{CO})_{g}$ (Rothberg et al., 1981).

The general dissociation mechanism yielding metal atoms from a host of metal carbonyls and related compounds is consistent with a two-electron exchange model proposed to explain the interaction of the carbonyl with the metastable rare gas state (Hartman et al., 1980). Conceptually, an electron from a high, filled molecular orbital of the 
carbonyl is first transfered to the ( $n$ )p hole of the metastable atom. The rare gas $(n+1)$ s electron is then returned to an unfilled orbital of the carbonyl. If the electron lands in an unbound molecular orbital, then ionization would be the result; ionization is presumably always a competing channel for energy disposal that we cannot monitor. From molecular orbital analyses for iron and nicksl carbonyl (Baerends and Ros, 1975), the metal atoms possess a formal positive charge in the molecular state, so the electron from the rare gas atom would land in an empty metal orbital. The orbital would be antibonding toward metal-ligand bonding and would lead to an instantaneous repulsive interaction leading in turn to a simultaneous dissolution of the metal-co bonds.

All the results from the rate model suggest that the dissociation is precisely such a simultaneous bond-breaking, snd appears to hold true even for cases such as ferrocene and $\mathrm{Mn}_{2}(\mathrm{CO})_{10}$. Ferrocene has a greatly lower energy dependence that all of the other cases which suggests that the only significant energy channels open are into the cyclopentadienyl and $\mathrm{Fe}$ atom translation modes, leaving initially cold cyclopentadienyl groups in terms of rotation and vibration.

The bond energies for the less well known carbonyls in some cases differ from the thermochemicai values, and the results place the total bond energies for $\mathrm{Fe}(\mathrm{CO})_{5}, \mathrm{Ni}(\mathrm{CO})_{4}$, $\mathrm{Cr}(\mathrm{CO})_{6}, \mathrm{CO}(\mathrm{CO})_{3} \mathrm{NO}, \mathrm{Mo}(\mathrm{CO})_{6}$, and $\mathrm{Fe}\left(\mathrm{C}_{5} \mathrm{H}_{5}\right)_{2}$ to be all on the order of $6 \mathrm{ev!} \mathrm{This} \mathrm{places} \mathrm{the} \mathrm{value} \mathrm{for} \mathrm{Mo(CO)_{6 }}$ several 
eV's lower than the thermochenical value of $9.7 \mathrm{eV}$. A test for the bimolecular nature of $\mathrm{Mo}(\mathrm{CO})_{6}$ dissociation would be necessary to confirm this lower value for the bond energy. At this point, between the low signal for the Mo lines observed and the ancient data for Mo, there is not a good value for the bond energy of $\mathrm{Mo}^{(\mathrm{CO})_{6}}$ from using the rate model.

Results from the pressure dependence of the long-lived states of iron suggest that intramultiplet quenching of metal states by the parent carbonyl is an extremely efficient process. The collisional process could compete for all states which are longer-lived than approximately $10^{-6}$ sesonds. In addition, for energy spacings of less than kT, a rapid Boltzmann population distribution is set up.

Metal atom reaction chemistry at this point is not tenable using the flowing afterglow technique due to the direct reaction of the reagent with the undissociated parent carbonyl and the very small concentrations of metal atoms produced. Perhaps a mass selection device such as a guadrupole field could be used to select the mass of the metal atom for reaction, but the concentrations woul be very low. In addition, the presence of high concentrations of carbonyls such as sticky iron carbonyl promises that it will be hard to keep the experiment clean and operating.

Although the flowlng afterglow technique may not be ideally suited for studying reaction chemistry, the carbonyls do seem to be good precursors for such work. 
Several reports have come out indicating that flash photolysis or intracavity laser operation produces band formation of small molecules such as wo from $W(C O)_{6}$ (Efremov et al., $1979)$ or a small, unidentified cr-containing compound from $\mathrm{Cr}\left(\mathrm{CO}_{6}\right.$ (Efremov et al., 1974).

The afterglow technique does have its limitations, the major one being the large error that is still inherent in the method. Until this error can be lessened, more precise information on dissociation mechanisms or bond energies will be unobtainable. The effects from fluctuations in flame intensities could be mitigated by signal averaging the lines of interest to average out these effects. More work on boosting gas phase concentrations could perhaps increase the intensities of the metal lines.

Finally, the one source of error that seems to be rapidJy disappearing is the accuracy with which the data on line transitions for metals is known. The National Bureau of standards is now making a systematic effort to improve the data for many transition metals, a task becoming ever easier as new laser systems and technologies make selective excitation of metal states more possible, thus replacing the rather archaic data presently available using methods containing incorrect temperature dependences and such. It would be worth returning to some of this data in the future for reanalysis when these newer values become available. 
References

Abel, E.W., and Stone, F.G.A. (1969). Quart. Rev. 23, 325. Baerends, E.J., and Ros, P. (1975). Holec. Phys. 30, 1735. Blackwe11, D.E., Ibbetson, P.A., Petford, A.D., and Shallis, M.J. (1979). Mon. Not. R. Ast. Soc. 186, 633 .

Blackwell, D.E., Ibbetson, P.A., Petford, A.D., and willis, R.B, (1976). Mon. Not. R. Ast, Soc. 177, 219.

Brennan, W.R., and Kistiakowsky, G.B. (1966). J. Chem. Phys. 44, 2695 .

Bridges, J.M., and Kornblith, R.L. (1974). Astrophys. T. $192,793$.

Calleăr, A.B. (1975). In Physical Chenistry, An Advanced Treatise, vol. 6B (B. Eyring, D. henderson, and W. Jost, eds.), Academic Press, New York.

Callear, A.B. (1978). Gas Kinetics and Energy Transfer 3, 82 .

Callear, A.B., and Oldman, R.J. (1966). Nature 210, 730.

Callear, A.B., and Oldman, R.J. (1967). Trans. Far. Soc. 63, 2888 .

Chang, R.S.F., and Setser, D.W. (1978). J. Chem. Phys. 69, 3885 .

Churchill, R.v. (1972). Operational Mathematics, 3rd ed., McGraw-Hill, New York.

Collins, C.B., and Robertson, พ.W. (1964), J. Chem. Phys. 40,301 .

Connor, J.A. (1977). Topics in Current Chemistry 71, 71.

Corliss, C.H. (1965), J. Res. 69A, 87.

Corliss, C.H., and Bozman, W.R. (1962). "Experimental Transition probabilities for Spectral Lines of Seventy Elements", oSkBS Hon. 53, U.S. Government Printing office, washington, D.C.

Corliss, C., and Sugar, J. (1977). J. Phys. Chen. Ref. Data 6. 1253 .

Corliss, C., and Sugar, J. (1981). J. Phys. Chen. Rei. Data 10,197 . 
Corliss, C.H., and Tech, J.I. (1968). "Oscillator Strengths and Transition Probabilities for 3288 Lines of Fe I." USNBS Won. 108, U.S. Government Printing office, washington, D.C.

Corliss, C.H., and Tech, J.L. (1976). J. Res. 80A, 787.

cotton, F.A., Fischer, A.K., and wilkinson, G. (1959). J Am. Chem. Soc. 81, 800 .

Cotton, F.A., and Wilkinson, G. (1972). Advanced Inorganic Chemistry, 3rd ed., Interscience, New York.

Dietz, T.G., Duncan, M.A., Powers, D.E., and Smalley, R.E. (1981). J. Chem Phys. 74, 6511.

Distefans. G. (1970). J Research 74A, 233.

Duncan, M.A., Dietz, T.G., and Smalley, R.E. (1979), J. Cher. Phys. 14, 415.

Efremov, Y.M., Gurvich, L.V., Savchenko, A.N., and Sviridenkov, E.A. (1979). Chem. Phys. Lett. 61, 179.

Efremov, Y.M., Samilova, A.N., and Gurvich, L.V. (1974). Opt. Spectrose. 36, 381 .

Engelking, P.C. (1980). (hen. Phys. Lett. 74, 207.

Fischer, A.K., Cotton, F.A., and Wilkinson, G. (1957). J. Amer. Chem. Soc. 79, 2044.

Forst, w. (1971). Chem. Rev. 71, 39.

Fuhr, J.R., Martin, G.A., Wiese, W.L., and Younger, S.M. (1981). J. Phys. Chen. Ref. Data 10, 305 .

Gerrity, D.P., Rothberg, L.J., and Vaida, V. (1980). Chem. Phys. Lett. 74,1 .

Golde, M.F., Ho, Y,-S., and Qgura, H. (1982). J. Chea. Phys. 76. 3535 .

Gundel, L.A., Setser, D.W., Clyne, M.A.A., Coxon, J.A., and Nip, W. (1976). J. Chen. Phys. 64, 4390.

Hartman, D.C. (1979). Ph.D. Thesis, University of California, Berkeley, California. (LBL Report 9819).

Hartman, D.C., Hollingsworth, W.E., and winn, J.S. (1980). J. Chen. Phys. 72, 833. 
Hartman, D.C., and Winn, J.S. (1978). J. Chen. Phys. 68, 2990 .

Hellner, L., Masanet, J., and Vermeil, C. (1979). Houveau Journal de Chimie 3,721 .

Hellner, L., Masanet, J., and Vermeil, C. (1981). Chem. Phys. Lett. 83, 474.

Horak, D.V. (1982). Ph.D. Thesis, University of California, Berkeley, California. (LBL Report 14612).

Horak, D.V., and Winn, J.S. (1982). J. Phys. Ches. (to be published).

Guber, K.P., and Herzberg, G. (1979). Molecular Spectra and molecular structure. IV: Constants of Diatomic molecules, Van Nostrand Reinhold, New York.

Ivanov, v.v. (1973). "Transfer of Radiation in Spectral Lines." OSHBS Spec. Pub. 385, U.S. Government printing OEfice, Washington, D.C.

Karny, Z., Naaman, R., and zare, R.N. (1978). Chem. Phys. Lett. 59, 33 .

King, D.L., and Setser, D.W. (1976). Ann. Rev. Phys. Chem. 27,407 .

Klabunde, K.J. (1980). In Reactive Internediates, vol. I (R.A. Abramovitch, ed.), Pienum, New York.

kobovitch, J., and Krenos, J. (1981). J. Chem. Phys. 74, 2662 .

Kolts, J.H., and Setser, D.w. (1978). J. Chem Phys. 68, $48 \varepsilon_{8}$.

Kolts, J.H., and Setser, D.W. (1979). In Reactive Intermediates in the Gas Phase (D.W. Setser, ed.), Academic Press, New York.

Lagowski, J.J. (1973). Hodern Inorganic Chemistry, Marcel Dekker, New York.

Leutwyler, S., and Even, U. (1981). Chen. Phys. Lett. 84, 188 .

Leutwyler, S., Even, U., and Jortner, J. (1980). Chen. Phys. Iett. 74,11 .

Lloyd, D.R., and Schlag, E.W. (1969). Inorg. Ches. 8, 2544. 
Mavrodineanu, R., and Boiteux, H. (1965). Plame Spectroscopy, Wiley, New York.

May, M., Richter, J., and Wichelmann, J. (1974). J. Astron. Astrophys. Suppl. 18, 405.

Moore, C.E. (1971). "Atomic Energy Levels", NSRDS-NBS 35, U.S. Government Printing office, Washington, D.C.

Muetterties, E.L. (1977). Science 196, 839.

Muetterties, E.L., Rhodin, T.N., Band, E., Brucker, C.F., and Pretzer, W.R. (1979). Chen. Rev. 79, 91.

Piper, L.G., Setser, D.W., and Clyne, M.A.A. (1975). J. Chem. Phys. 63, 5018.

Prince, J.F., Collins, C.B., and Robertson, W.W. (1964). J. Chem. Phys. 40, 2619.

Reader, J., and Sugar, J. (1975). J. Phys. Chen. Ref. Data 4. 353.

Rothberg, L.J., Gerrity, D.P., and Vaida, V. (1981). J. Chem. Phys. 74, 2218.

Schaefer, H.F. (1977). Accts. Chem. Res. 10, 287.

Snyder, H.L, Parr, T.P., Smith, B.T., and Mar.in, R.M. (1980). Chem. Phys. Letr. 73, 487.

Stedman, D.H., and Setser, D.W. (1970). J. Chem. Phys. 52, 3956.

Stedman, D.H., and Setser, D.W. (1972). Prog. React. Kinet. 6. 193.

Sugar, J., and Corliss, C. (1977). J. Phys. Chem. Ref. Data 6,317 .

Sugar, J., and Corliss, C. (1981). J. Phys. Chem. Ref. Data 10, 1097 .

Trainor, D.W., and Mani, S.A. (1978). J. Chem. Phys. 68, 5481 .

Vaida, V., Cooper, N.J., Henley, R.J., and Leopold, D.G. (1981). J. A. Chem. Soc. 103, 7022.

Velazco, J.E., Kolts, J.H., and Setser, D.W. (i978). J. Chen. Phys. 69, 4357.

Welch, J.A., Peters, K.S., and Vaida, V. (1982). J. Phys. Chem. 86, 1941 . 
Wiese, W.L., Smith, M.W., and Miles, B.M. (1969). "Atomic Transition Probabilities", vol. 2, NSRDS-NBS 22, U.S. Government Printing office, Washington, D.C.

Wolnik, S.J., Berthel, R.O., and Wares, G.W. (1970). Astrophys. J. 162, 1037 .

Yardley, J.T. (1980). Introduction to Molecular Energy Transfer, Academic Press, New York.

Younger, S.M., Fuhr, J.R., Martin, G.A., and Wiese, W.L. (1978). J. Phys. Chem. Ref. Data 7, 495. 
Table I

Rare Gas Metastable state Energies

\begin{tabular}{lccc} 
Rare gas & State & Energy $\left(\mathrm{cm}^{-1}\right)$ & Energy(eV) \\
\cline { 2 - 4 } Helium & ${ }^{3} \mathrm{~S}_{1}$ & 159850 & 19.82 \\
& ${ }^{1} \mathrm{~S}_{0}$ & 166272 & 20.62 \\
Neon & ${ }^{3} \mathrm{P}_{2}$ & 134044 & 16.62 \\
& ${ }^{3} \mathrm{P}_{0}$ & 134821 & 16.72 \\
Argon & ${ }^{3} \mathrm{P}_{2}$ & 93144 & 11.55 \\
& ${ }^{3} \mathrm{P}_{0}$ & 94554 & 11.72 \\
& ${ }^{3} \mathrm{P}_{2}$ & 79973 & 9.92 \\
Krypton & ${ }^{3} \mathrm{~F}_{0}$ & 85192 & 10.56 \\
& & & 8.32 \\
& ${ }^{3} \mathrm{P}_{2}$ & 67068 & 9.45 \\
& ${ }^{3} \mathrm{P}_{0}$ & 76197 &
\end{tabular}


Table II

Physical Properties for some Metal Carbonyls

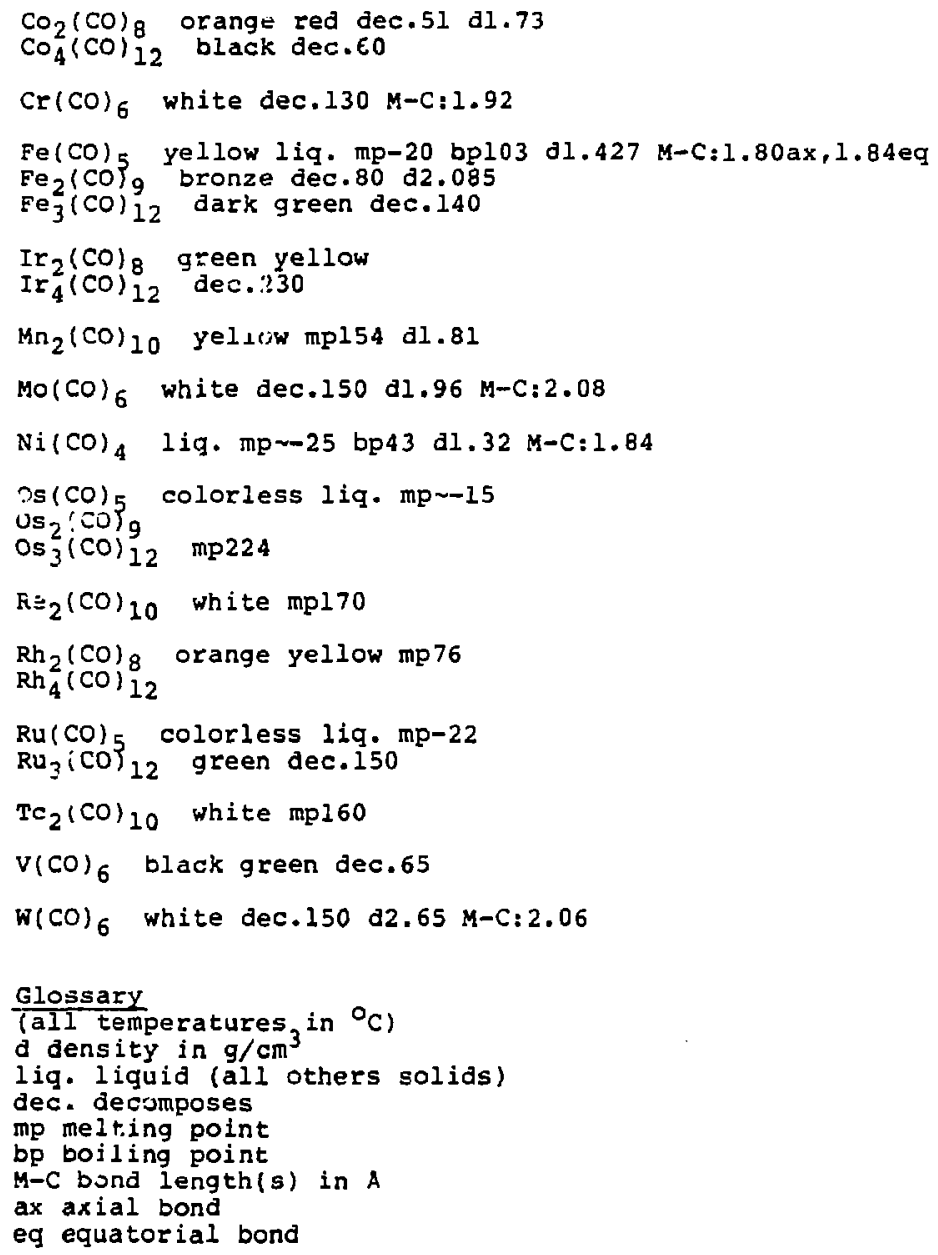




\section{Table III}

The Observed Long-lived Multiplets of Fe

$$
\begin{aligned}
& \text { Spacing }\left(\mathrm{cm}^{-1}\right) \quad \underline{I(\mathrm{sec})} \text { Intensity Ratios } \\
& \text { I }
\end{aligned}
$$

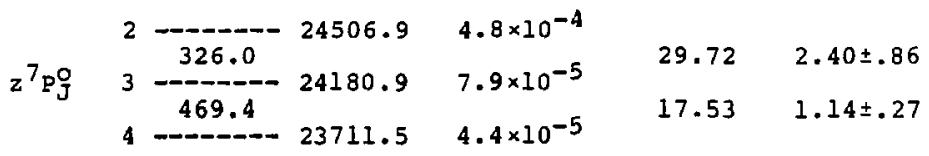

$$
\begin{aligned}
& 0 \text { - } 25.623270 .4 \text { metastable } \\
& 1 \text { - } 23244.8 \quad 6.0 \times 10^{-5} \\
& 52.3-23192.5 \quad 4.3 \times 10^{-5} \\
& z^{7} \mathrm{~F}_{\mathrm{J}} \quad 3 \quad 81.6 \quad 23110.9 \quad 3.3 \times 10^{-5} \quad 1.90 \quad 1.59 \pm .20 \\
& 114.2 \quad 2.03 \quad 2.04 \pm .10 \\
& 4 \frac{114.2}{150.8} 22996.7 \quad 2.9 \times 10^{-5} \\
& 5 \text { - } \\
& 6 \text { - } 195.5 \text { 22650.4 } 2.3 \times 10^{-3} \\
& 1.79 \quad 1.77 \pm .10 \\
& 0.04 \\
& 1+20019.6 \quad 4.3 \times 10^{-4} \\
& 2 \frac{107.1}{155.5} 19912.5 \quad 3.2 \times 10^{-4} \\
& z^{7} D_{j} \quad 3 \frac{155.5}{194.6} 19757.0 \quad 2.2 \times 10^{-4} \\
& 4 \text { - } 19562.4 \quad 2.0 \times 10^{-4} \\
& 5 \text { [11.5 } 19350.9 \quad 5.9 \times 10^{-4} \\
& 2.32 \\
& 3.06 \quad 3.30 \\
& 2.93 \quad 2.34 \\
& 0.93
\end{aligned}
$$




\section{Table IV}

The Observed Long-lived Multiplets of $N i$

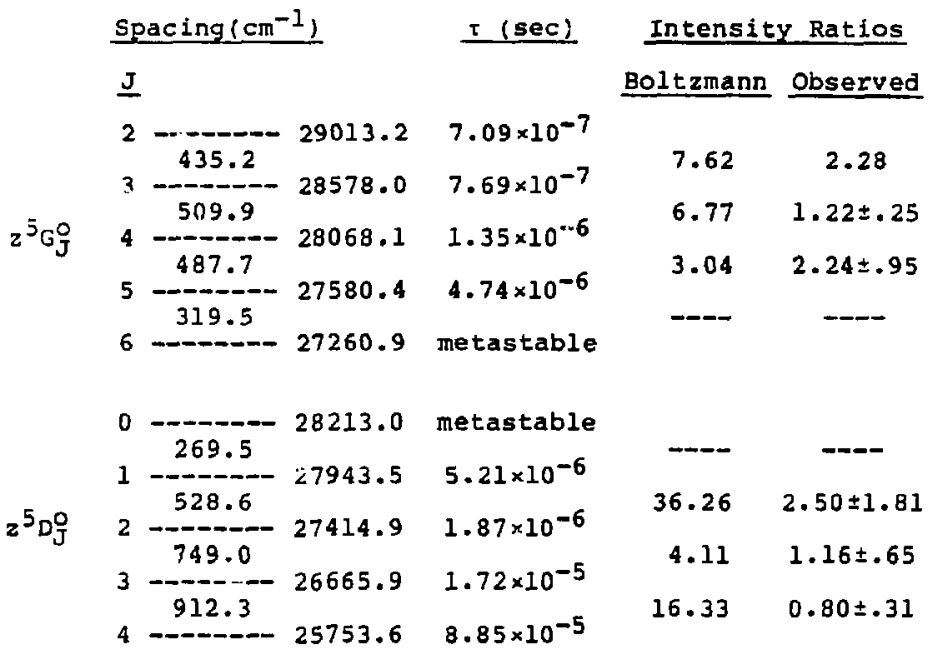




\author{
Table V \\ Carbony1 Bond Energies (in ev)
}

Thermochemical Spectral Limit Rate Mode ${ }^{1}$

$\begin{array}{lccc}\mathrm{Cr}(\mathrm{CO})_{6} & 6.7 & <7.6 & 6.0 \\ \mathrm{Mo}(\mathrm{CO})_{6} & 9.4 & >7.1 & (1.9) \\ \mathrm{W}(\mathrm{CO})_{6} & 11.1 & <14.5 & \ldots \\ \mathrm{Mn}_{2}(\mathrm{CO})_{10} & 11.1 & <7.3 \\ \mathrm{Co}(\mathrm{CO})_{3} \mathrm{NO} & --- & <7.4 & 6.3 \\ \mathrm{Fe}\left(\mathrm{C}_{5} \mathrm{H}_{5}\right)_{2} & 6.2 & \end{array}$

I parentheses indicate values with low to no confidence levels. 
Appendix

\section{Computer Program Listings}

\section{A. Program "NICOLET->PET"}

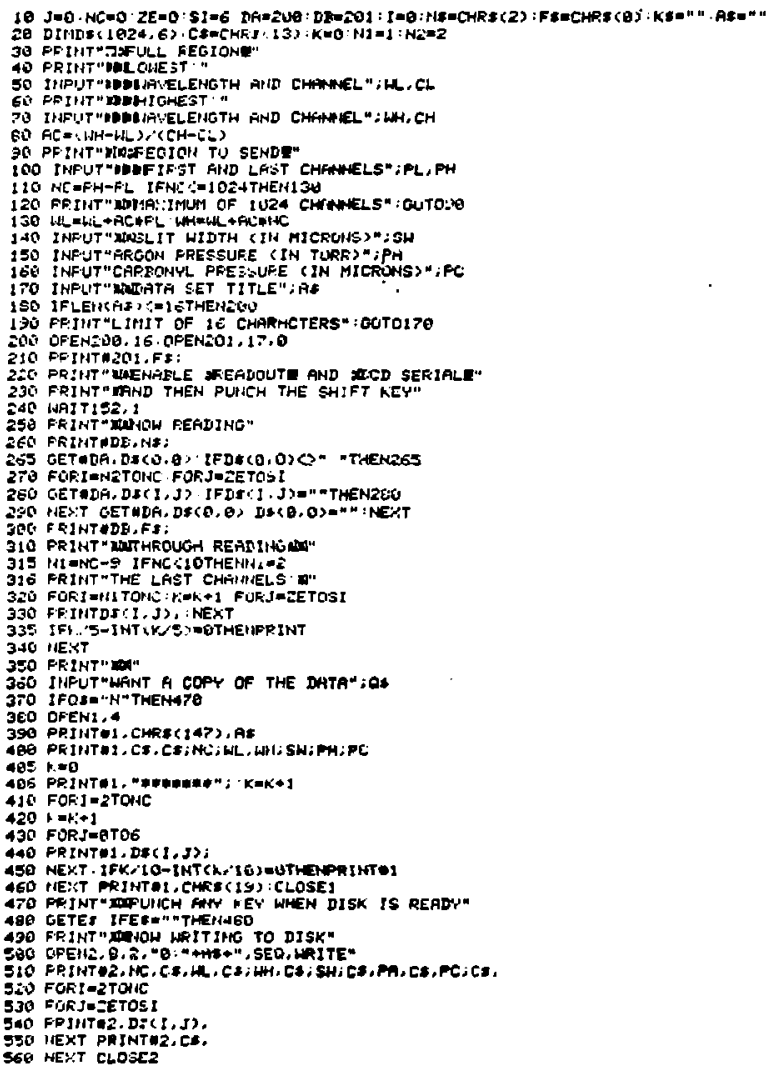




\section{B. Program "Data Process"}

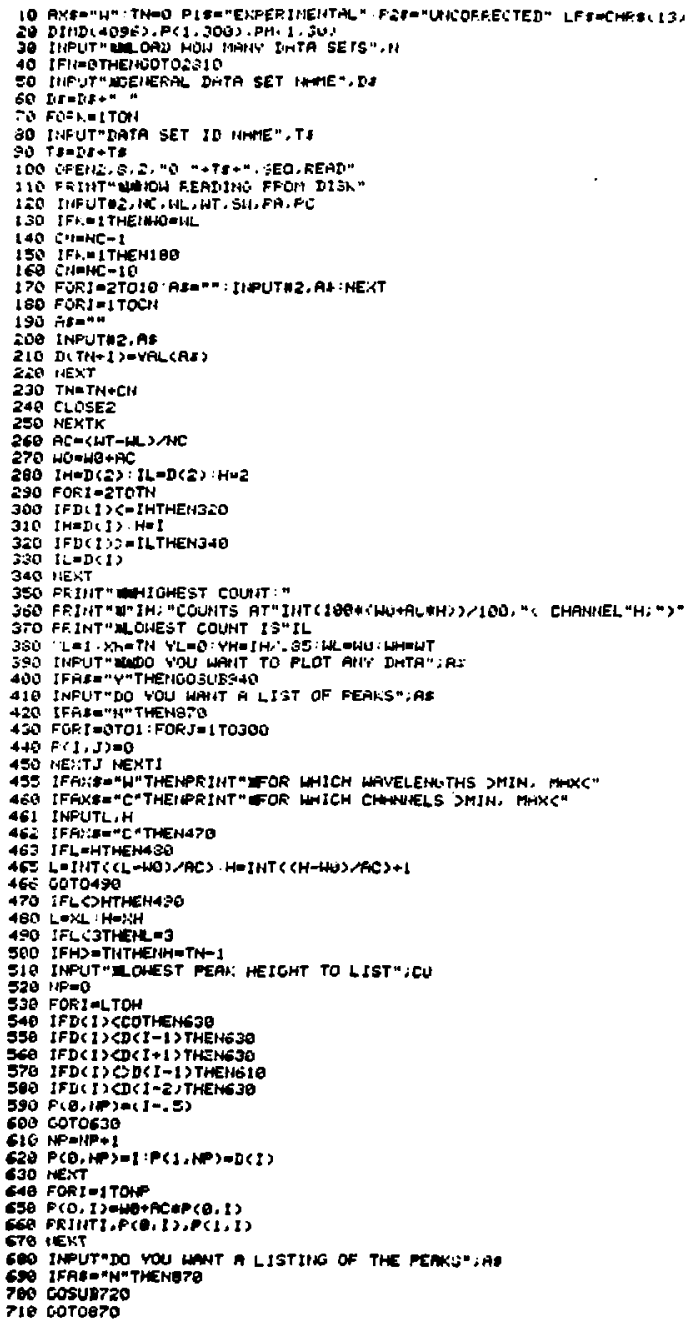




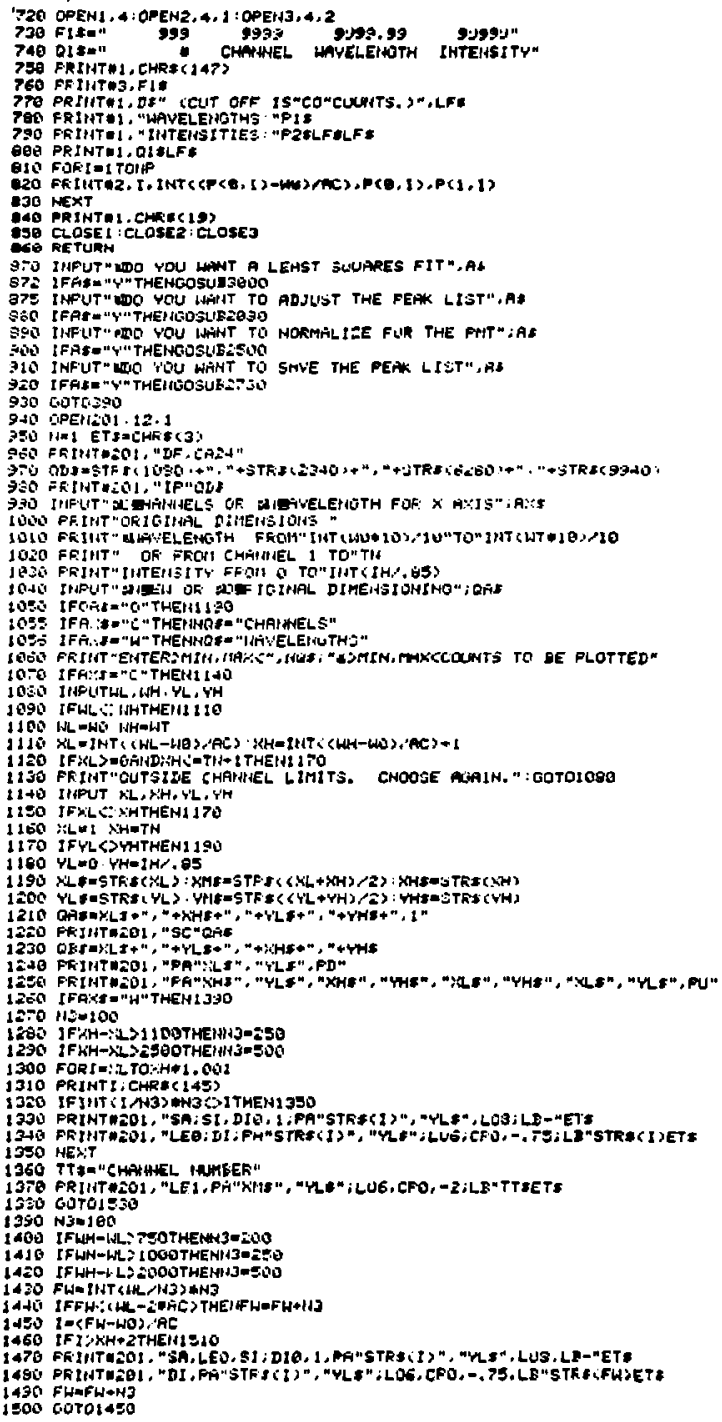




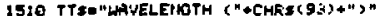

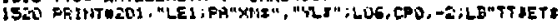

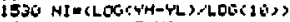

iste FRint -InT (H, )

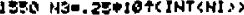

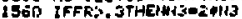

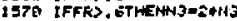

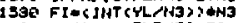

1590 [FF] SYYLTHEIF [OFIOHI

1600 IFF I; YHTHEN1680

1610 FRITTWEO, "LEO,SI, DI ; PA"MLS", "STRELFI, , LOSILI-"ETE

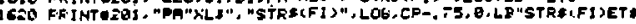

$1630 \mathrm{FI} * \mathrm{FI} \rightarrow \mathrm{N}_{3}$

1640 corol 500

1650 TT\&" "IITENSITY"

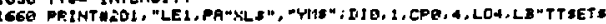

1670 SRIHT" THE CURREHT PLOT TITLE IS:"i IPS

1690 INPUT"DO YOU WFITT THE SYHE TITLE MOW"; MS

1690 IFAS $=$ "YMTHEHSTAS

1700 PRIIT IINUT TITLE OF PLOT

1710 FRINT"UFPER/LOWEP CASE. ti"

17:0 PRIHT "SUFER-,SUS- SLRIPTS J'ף"

1730 INFUTTFS

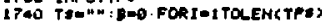

1730 Istmidectre.1,1)

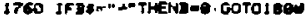

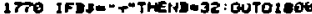

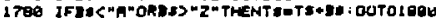

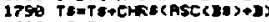

1000 NEXT

1810 LHE. 300. LHE. 456

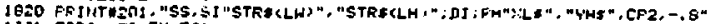

16:" FOFJ-1TOLENSTS

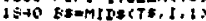

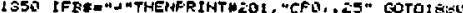

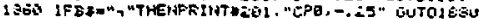

1370 PEINTW201, HLOZ, LE" EEET

ISEO MER:T

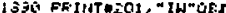

IOGO FFIIIT 201 , "LT. SI"

1310 IHFUT "S STAF THD EHS: LF FLUT SEOHEHT".CU.C:

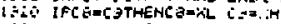

1 ast FOR $1=5$ aTOCs

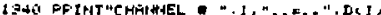

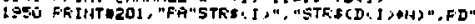

1000 NENT I

1970 FOIITTE201, "Pu"

1 SEO INFUT "DD YOU WHNT TO REPLOT A SEUMEMT" PS

1390 IFF SM "M"THEN

20.Ü INPUT"DO YOU WFHT ANOTHER PLUT",AJ

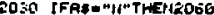

2OAB PRIHTU201, "FG"

Doso gotos:e

ZOSO ELOSEZO1.12

3OTO RETURN

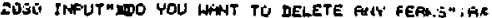

EOgQ TFAFE "IT"THENE1 10

2100 IHFUTM MELLTE PEFH. "iDF

2110 JFIP-OTHENE 160

2120 JFDFEMTHEHS16t

ijo FOFIOTPa1TOHF

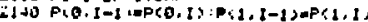

S150 NE:ST

3180 isfate-1

2I 90 GoTOzion

ALO IHPUT"DO YOU WHATT TO ADD ANY FEAK', RE

2EOO ITHPUT"PEW WHIVELEIHGTH, IHTEHSITYM, TH, HI

¿2lO IFTHEOTHENR320

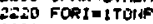

2230 IFIHCP(O. I) TMENASSO

5240 $1 \mathrm{EST}$

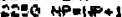

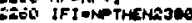

Xo FaRJAT-1TOJSTEP-1

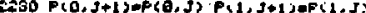

2890 IEXT

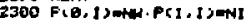

2310 00T02200

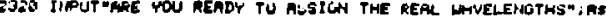

2330 JFAL WN"THENB 400 


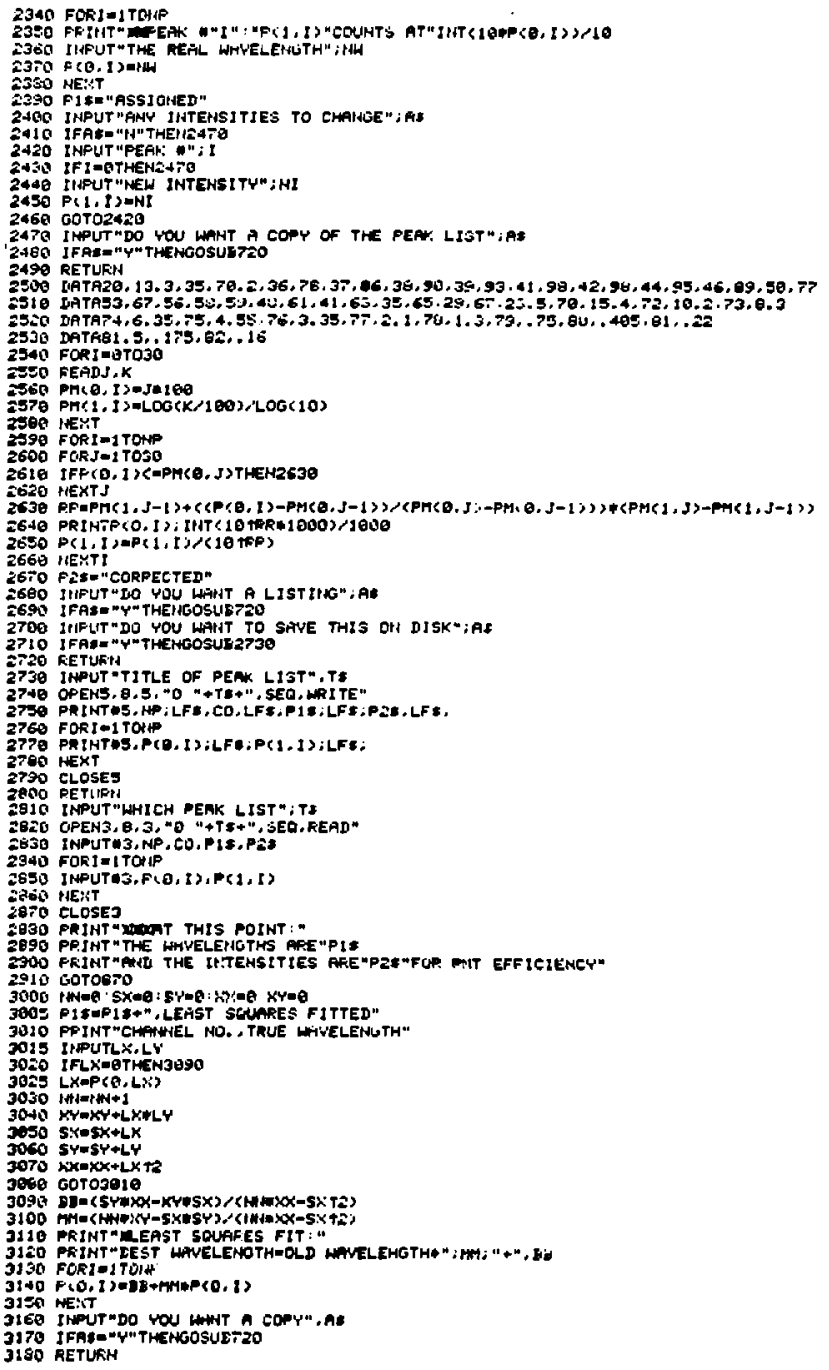


100-240 Reading spectrum from disk copy from "NICOLET->PET" 260-370 Determination of highest and lowest intensities 430-670 Peak finding over a cutoff of CO

720-8c0 Peak printing subroutine

940-2070 Subroutine: spectrum plotting

2080-2170 Peak deletion option

2I80-2310 Peak addition option

2320-2390 Peak assignment

2420-2460 Intensity change option

2500-2670 PMT efficiency correction

2730-2800 Disk save of peak list

2810-2910 Reading of peak list from disk

3005-3180 Subroutine: Least squares fit of selected lines 


\section{Program "P \& R PLOTTER"}

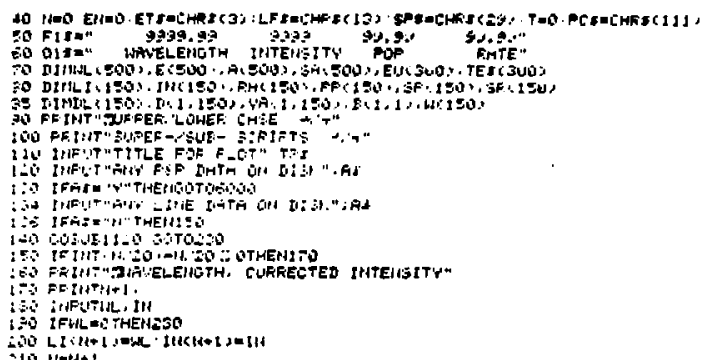

210 limt

30 gotolso

30 FRINT"FREPFPE DISK RECOKD OF METAL EILERU W LEVELS AND LINES"

$\$ 40$ OOSUET 000

ISO IUfUT" "HIOHEST POSSIBLE E LE HELS" I HE

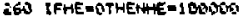

365 IIFUT"SUFFFESS HAFD COPY", HST

206 IFHSE" "Y"THEHSDE

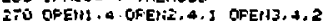

IBO FRIIT Q1. SFE,SFS.

200 FÜFM=1 TULEHICTPS,

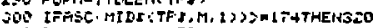

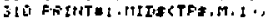

SOH HE: TT

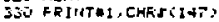

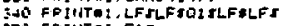

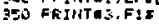

5isi $410100 \cdot r j=100 \cdot 42=-100 \cdot 440-100$

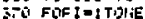

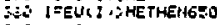

$3 \rightarrow$ indit

IF IFH'SSE"Y"THEHA1a

+\$D FRIHTA1. Ii"*",EUCIS

+10 EOF:T=1TOHL

+20 IFE ( $J, \therefore$ ITHEHSOO

+30 FOFKeI TOK.

t40 JFL $\{(K) \circlearrowleft H L(S)$ THENH +90

$\$ 50,14 \mathrm{H}=12 \mathrm{~W}+1$

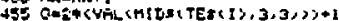

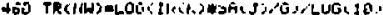

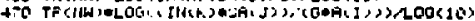

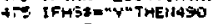

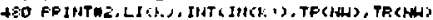

+90 NE:T

SOO NES:T

S10 IFINS OTHEIS30

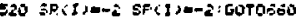

Sig IFIAUI THERESO

5.0 Gosurt310 GoTOSTo

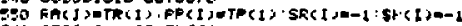

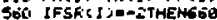

STO JFSR ( I) $=-1$ THEI WOOO

ST JFHSTM"Y"THETEOO

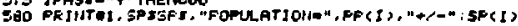

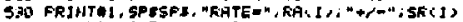

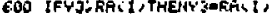

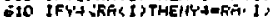

SDO IFYIXEPI I THEIYIDFFid,

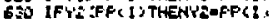

Ga coróseo

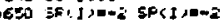

CEO HE:TT

GE TFHESOMYNTHEHWO

$\rightarrow 0$ FinJitet, CHFig (19)

teO CLOSES ClOSEZ CLOSE

G9a Fonmi i Tone

TOW IFEUCMSS HETHENT30

710 IFSP $(M)<-1$ THEM 30

$7: \theta$ WHDELCM)

$7 \geq 0$ IHE:T 


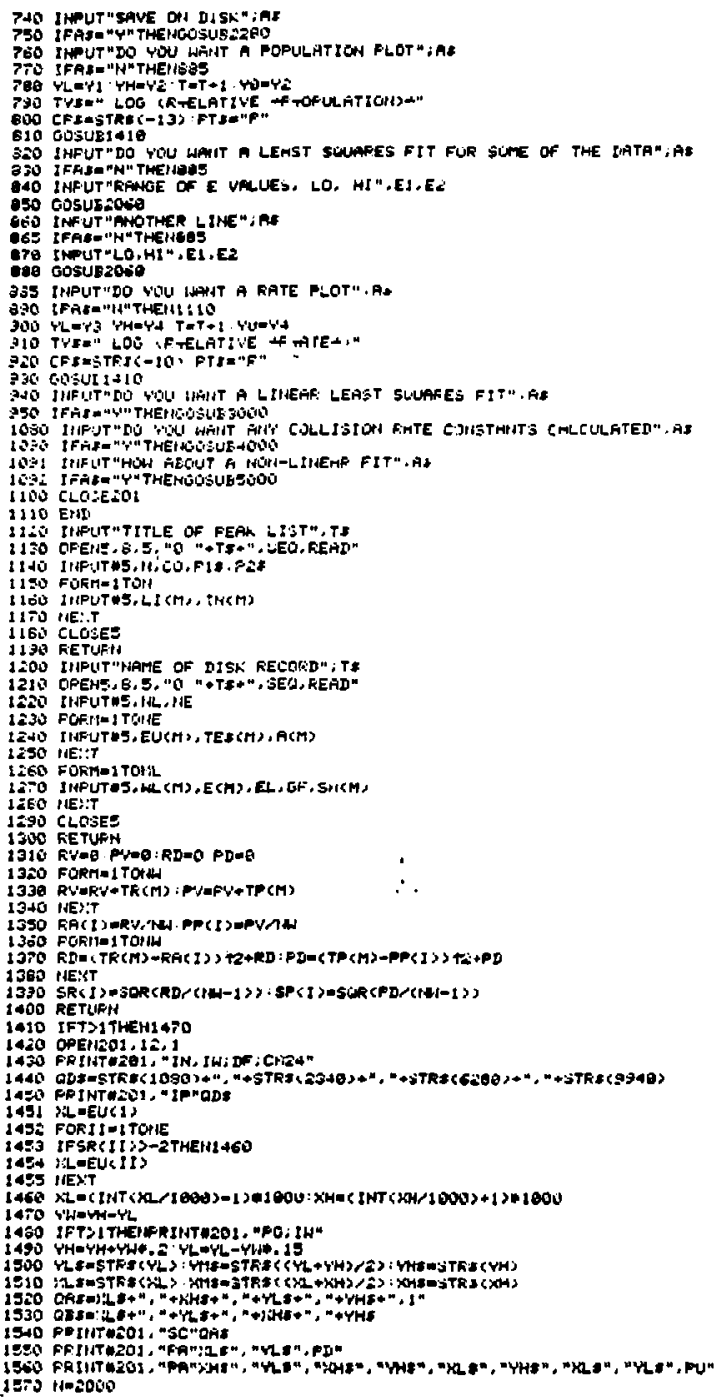




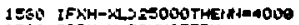

IESt) FORHIXLTOULSTEPH

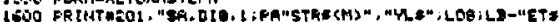

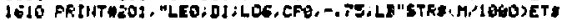

IE: 1 inXT

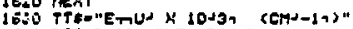

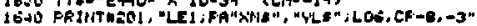

1650 cosutisio

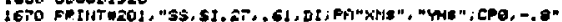

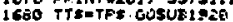

I:50 FEIIITUEOL, "SA, LEO, DI : LOS"

1700 FQRMEOTOTHT KYH-YL?

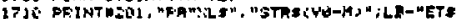

I7:0 IFMEOTHEAPF IATESO1. "CP-, TS, Q. LR"STRE<MIET:

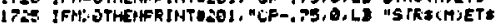

1730 +IE:;T

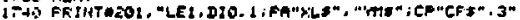

ITES TTSOTYS

TES GOSUE 19:0

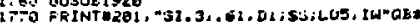

ITEO FORMEITORE

1700 IFSR (M)-ETHEN1500

1800 IFPTS COPHTHEH1 1820

tato VIJPP (H) : SOESP (M) : CUTa103t

1020 YHERASH) : SOMSP (n)

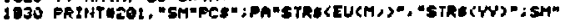

1040 LFSDE-ithertisea

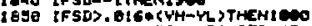

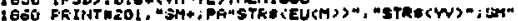

tera Dototsog

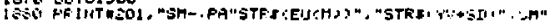

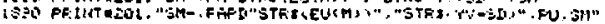

i:

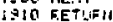

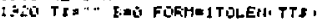

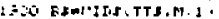

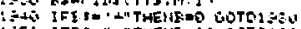

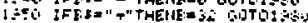

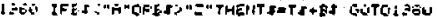

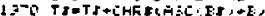

$\therefore$ I.E.T

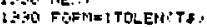

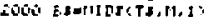

2015 JFFI

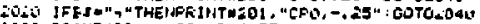

QQSO PRIHT Q2O1, "LDE, LE*BAETS

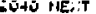

:OFO FETUFr

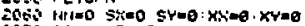

sofs FOFHE I TOHE

¿080 IFEUM (1) CE: THEM 1 Ë

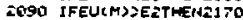

2100 IFSEINSC-1TMETIE160

110 Plliontd+?

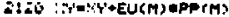

125 Gi:-3it+EU(N)

2140 SHeST+FF (M)

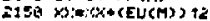

2160 IEKT

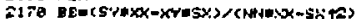

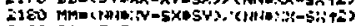

2190 FRIIT"THE SLOFE OF THE LINE is": iN

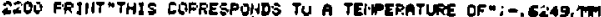

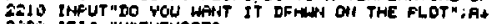

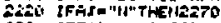

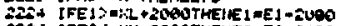

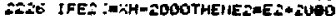

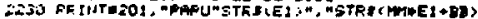

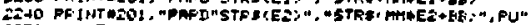

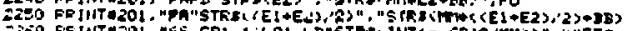

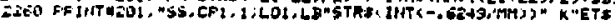

ENTI EETURI

2ESS IHPUT"TITLE FON DISH: RECOF" ; TDS

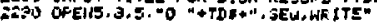

t5iv $\mathrm{V}=0$

¿310 FOFM"1TDAE

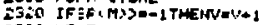

$\$ 3 \$ 0$ IIE

E+Q FRIMTEG, V,LFE:

2050 FCFME 1 TOHE

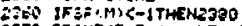

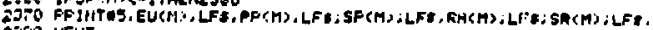

\&E0 Hext 


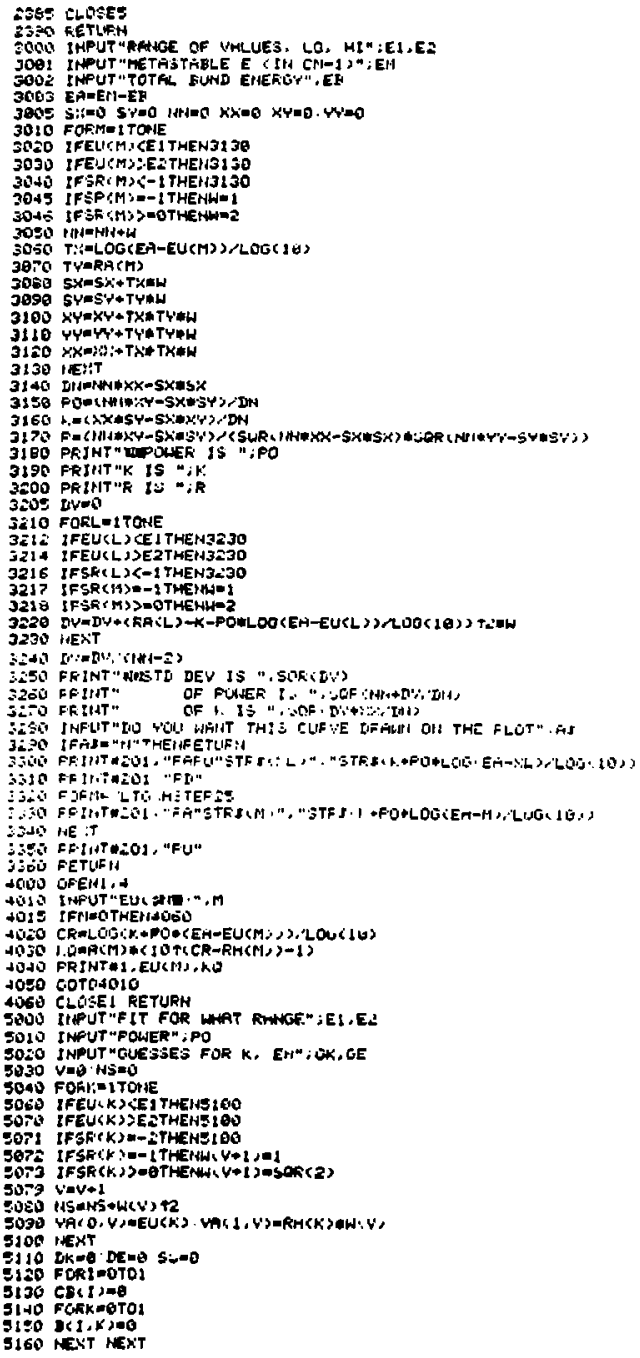




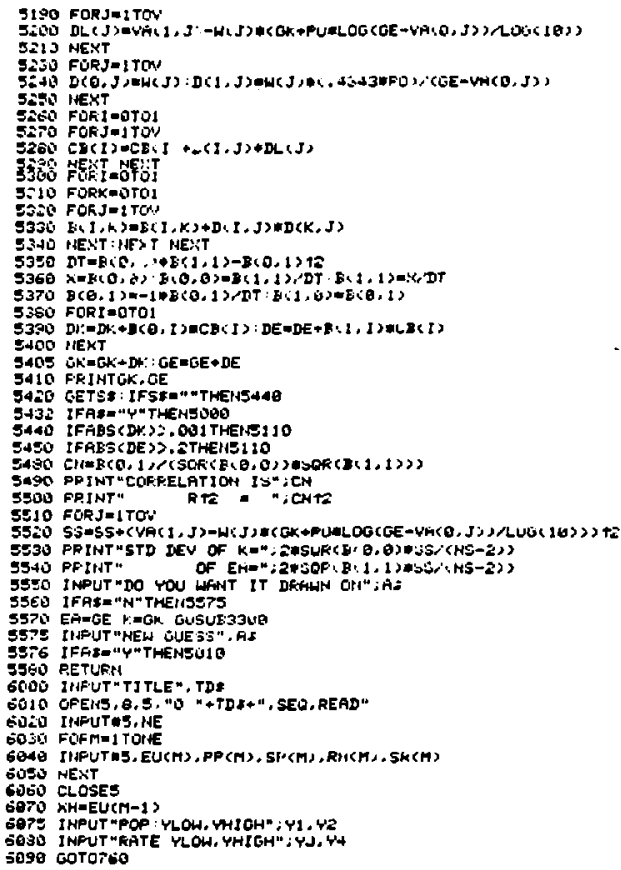

90-110 Formatting of title of plot

150-220 Manual input of wavelengths and intensities

270-680 printing of rate and population calculations

455-470 Rate and population equations

600-640 Finding the extremes of populations and rates

780-800 Set-up for population plot

900-920 Set-up for rate plot

1120-1190 Subroutine: Recall of peak list from disk

1200-1300 Subroutine: Reading of metal lines data from disk

1310-1400 Subroutine: Calculation of standard deviation for

the rate and population averages for each energy level

1420-1910 Subroutine: Population and Rate plotting

1920-2050 Subroutine: Printing the plot title

2060-2270 Subroutine: Calculation and plotting of tempera-

ture

2280-2390 Subroutine: Copy of $P \& R$ data on the disk

3000-3270 Subroutine: Linear least squares routine for best rate curve

3300-3360 Subroutine: Printing of difference between calculated and observed rates 
5000-5580 Subroutine: Nonlinear least squares $f$ it for rate plots 6000-6090 Subroutine: Reading $P$ \& data from disk 